Issued by Sandia National Laboratories, operated for the United States Department of Energy by Sandia Corporation.

NOTICE: This report was prepared as an account of work sponsored by an agency of the United States Government. Neither the United States Government, nor any agency thereof, nor any of their employees, nor any of their contractors, subcontractors, or their employees, make any warranty, express or implied, or assume any legal liability or responsibility for the accuracy, completeness, or usefulness of any information, apparatus, product, or process disclosed, or represent that its use would not infringe privately owned rights. Reference herein to any specific commercial product, process, or service by trade name, trademark, manufacturer, or otherwise, does not necessarily constitute or imply its endorsement, recommendation, or favoring by the United States Government, any agency thereof, or any of their contractors or subcontractors. The views and opinions expressed herein do not necessarily state or reflect those of the United States Government, any agency thereof, or any of their contractors.

Printed in the United States of America. This report has been reproduced directly from the best available copy.

Available to DOE and DOE contractors from

Office of Scientific and Technical Information

P.O. Box 62

Oak Ridge, TN 37831

Prices available from (703) 605-6000

Web site: http:/www.ntis.gov/ordering.htm

Available to the public from

National Technical Information Service

U.S. Department of Commerce

5285 Port Royal Rd

Springfield, VA 22161

NTIS price codes

Printed copy: A04

Microfiche copy: A01

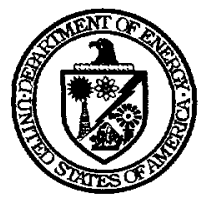




\section{DISCLAIMER}

\section{Portions of this document may be illegible in electronic image products. Images are produced from the best available original document.}


SAND2000-8577

Unlimited Release

Printed March 2000

\title{
STABILITY OF QUASI-STEADY DEFLAGRATIONS IN CONFINED POROUS ENERGETIC MATERIALS
}

\author{
Alexander M. Telengator ${ }^{\dagger}$, Stephen B. Margolis ${ }^{\ddagger}$ and Forman A. Williams ${ }^{\dagger}$ \\ tDepartment of Mechanical and Aerospace Engineering \\ University of California, San Diego \\ La Jolla, California 92093-0411 USA

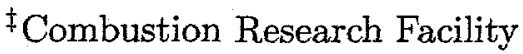 \\ Sandia National Laboratories \\ Livermore, California 94551-0969 USA
}

\begin{abstract}
Previous analyses have shown that unconfined deflagrations propagating through both porous and nonporous energetic materials can exhibit a thermal/diffusive instability that corresponds to the onset of various oscillatory modes of combustion. For porous materials, two-phase-flow effects, associated with the motion of the gas products relative to the condensed material, play a significant role that can shift stability boundaries with respect to those associated with the nonporous problem. In the present work, additional significant effects are shown to be associated with confinement, which produces an overpressure in the burned-gas region that leads to reversal of the gas flow and hence partial permeation of the hot gases into the unburned porous material. This results in a superadiabatic effect that increases the combustion temperature and, consequently, the burning rate. Under the assumption of gas-phase quasi-steadiness, an asymptotic model is presented that facilitates a perturbation analysis of both the basic solution, corresponding to a steadily propagating planar combustion wave, and its stability. The neutral stability boundaries collapse to the previous results in the absence of confinement, but different trends arising from the presence of the gas-permeation layer are predicted for the confined problem. Whereas two-phase-flow effects are generally destabilizing in the unconfined geometry, the effects of increasing overpressure and hence combustion temperature associated with confinement are shown to be generally stabilizing with respect to thermal/diffusive instability, analogous to the effects of decreasing heat losses on combustion temperature and stability in single-phase deflagrations.
\end{abstract}





\section{STABILITY OF QUASI-STEADY DEFLAGRATIONS IN CONFINED POROUS ENERGETIC MATERIALS}

\section{Introduction}

The combustion behavior of energetic materials is of critical importance in a number of applications arising in propulsion, pyrotechnics and gas generation. Widely studied examples of such materials are the nitramine propellants HMX and RDX, which are frequently used in various rocket-motor formulations and explosive devices. These materials, once manufactured and placed in a system, may be stored for considerable lengths of times. During that time, they may become chemically degraded and develop significant porosities arising from inherent long-term metastability in the chemical formulation and/or external influences such as an inadvertent exposure to an abnormal thermal environment. Once degraded, the combustion behavior will be affected because of changes in energy density, chemical composition and two-phase flow effects within the now-porous material, all of which thus affect the performance and safety characteristics of the system. These considerations have served to motivate a number of recent theoretical studies on the combustion of porous energetic materials (cf. [1-12]). Most of these have considered unconfined materials, but in this and a previous study [12], we consider the combustion behavior of such materials under confinement.

The description of combustion in a porous material requires a two-phase-flow description that is generally quite complex (cf. [13]). Moreover, descriptions of combustion and other processes in a multiphase continuum often vary because of the specification of various constitutive relations that are generally required to give a closed mathematical model. Nonetheless, depending on the actual problem, various simplifications can often be made. Those that are appropriate for the present problem of interest are described in the following sections, but all formulations must allow for relative motion between coexisting phases to capture true two-phase-flow effects that are present in degraded materials. This becomes especially critical in the confined geometry of interest here, where the presence of confinement results in a significant overpressure in the burned region that in turn causes a reversal of the flow velocity and allows hot gases to permeate the unburned material. Such gas permeation is generally attributed to a significant increase in the burning rate (cf. $[1,6,12,14]$ ) that is usually associated with the transition to a fast burning regime, often referred to as convective burning, in which the above process serves to preheat the unburned porous solid and thus enhance both the combustion temperature and the burning rate, even when porosities are relatively small.

Attempts to analyze the combustion stability of porous energetic solids are relatively recent. In two such studies $[8,9]$, an unconfined geometry was considered such that pressure variations could reasonably be neglected. This and various other reasonable approximations led to the derivation of 
an asymptotic model for combustion in the limit of large overall activation energy. That formulation admitted an explicit basic solution corresponding to a steadily propagating planar combustion wave, thus facilitating a subsequent analysis of its linear stability. It was found, as in the nonporous case, that there existed an oscillatory neutral stability boundary such that for sufficiently large values of an overall activation-energy parameter, stability of the basic solution was lost to a nonsteady, nonplanar form of propagation. In addition, it was determined that in the realistic limit of small gas-to-solid density and thermal-conductivity ratios, the shifts in the stability boundary relative to the nonporous problem were essentially proportional to $\alpha_{s}\left(1-\alpha_{s}\right)^{-1}$, where $\alpha_{s}$ is the gas-phase volume fraction, or porosity, of the solid.

The present work is a generalization of these recent stability analyses to a confined geometry in which even the basic solution is subject to the overpressure and gas-permeation effects described above [12]. Although the inherent unsteadiness of the confined problem can be addressed with the reasonable assumption of a quasi-steady gas phase (cf. $[9,12,15])$, it is necessary to relax the constant-pressure assumption in order to account for the pressure-driven gas-permeation effect. In this and the previous analysis [12] of the basic solution, we thus adopt a Darcy representation of the relationship between gas velocity and pressure gradient in the solid/gas region. While this is a simplification relative to a full consideration of gas-phase momentum in this region, it nonetheless still results in a more complex wave structure by introducing, in the realistic limit of small permeability, a gas-permeation boundary layer within the solid/gas preheat zone where both pressure and gas velocity vary significantly. Consequently, perturbation expansions of both the basic and perturbed solutions, the temporal growth or decay of which determine the stability of the basic solution, must be introduced at an earlier stage in the analysis than was required for the unconfined studies $[8,9]$. The final result appears in the form of a dispersion relation for the perturbed growth rate relative to the leading-order dispersion relation associated with the nonporous, unconfined problem. The subsequent analysis of this relation thus determines the shift in the neutral stability boundaries arising from both porosity and confinement, allowing the prediction of significant trends associated with the latter.

\section{Formulation}

As in previous studies [7-12], we assume an overall reaction process of the form $R(s) \rightarrow R(l) \rightarrow$ $P(g)$, where the first step represents the melting of the solid, and the second step denotes a one-step exothermic reaction in which the liquid-phase material is converted to burned gaseous products. A schematic illustration of combustion-wave propagation in the confined geometry of interest is then shown in Figure 1a. The unburned porous solid and final gas products are separated by a combustion wave propagating from right to left. Although the problem is confined, we assume that the right and left boundaries are sufficiently far away relative to the width of the flame region 
that the primary effect of confinement is a difference between the upstream and downstream values of pressure. Since this pressure difference, or overpressure, is generally positive, there is, for typically small permeabilities and sufficiently large overpressures, a thin sublayer embedded within the solid/gas region where a large pressure gradient drives a reverse gas flow into the pores of the unburned solid. Specifically, proceeding from left to right, the wave structure is comprised of the solid/gas preheat region, the aforementioned gas-permeation boundary layer in which the gas velocity and pressure vary significantly, the melting surface across which the solid material is converted to a liquid, a liquid/gas preheat region, the thin reaction zone where exothermic conversion of the liquid material to gaseous products occurs, and finally, the confined burned-gas region.

In what follows, the subscripts $s, l$ and $g$ denote solid-, liquid- and gas-phase quantities, respectively, and the subscripts $u$ and $b$ indicate unburned and burned values. Dimensional quantities are denoted by tildes, and the spatial coordinates are denoted by $\left(\tilde{x}_{1}, \tilde{x}_{2}, \tilde{x}_{3}\right)$, the last of which is the longitudinal coordinate in the direction of the burned gas. In order to simplify the analysis without compromising the fundamental two-phase-flow aspects of the problem, we adopt several assumptions that were also used in various earlier studies (cf. $[9,12,16])$. In particular, we assume, as indicated above, that the far-field gas pressure is quasi-static (eventually we shall consider the gas phase to be completely quasi-steady with respect to the solid), that there is temperature equilibrium between coexisting phases, and that the combustion wave is at most weakly nonplanar so that transverse gas-phase velocity components are small compared with the longitudinal component $\tilde{u}_{3}$. Other approximations will be indicated as they are introduced.

With the melting surface denoted by $\tilde{x}_{3}=\tilde{x}_{m}$ and the gas-phase volume fraction by $\alpha$, overall mass conservation is given by

$$
\frac{\partial \tilde{\rho}_{g}}{\partial \tilde{t}}+\frac{\partial}{\partial \tilde{x}_{3}}\left(\tilde{\rho}_{g} \tilde{u}_{g}\right)=0, \quad \tilde{x}_{3}<\tilde{x}_{m}
$$

and

$$
\frac{\partial}{\partial \tilde{t}}\left[(1-\alpha) \tilde{\rho}_{l}+\alpha \tilde{\rho}_{g}\right]+\frac{\partial}{\partial \tilde{x}_{3}}\left[(1-\alpha) \tilde{\rho}_{l} \tilde{u}_{l}+\alpha \tilde{\rho}_{g} \tilde{u}_{g}\right]=0, \quad \tilde{x}_{3}>\tilde{x}_{m}
$$

where $\tilde{\rho}$ is density and $\tilde{\rho}_{s}$ and $\tilde{\rho}_{l}$ are assumed constant. Also, it has been assumed that $\tilde{u}_{s}=0$ in the adopted coordinate system and thus solid-phase continuity simply implies that $\alpha$ is independent of time in the solid/gas region (for simplicity, we assume a constant porosity $\alpha=\alpha_{s}$ for the solid). Consequently, solid-phase continuity becomes trivial and overall continuity in the solid/gas region, Eq. (2.1), becomes equivalent to gas-phase continuity there. Liquid-phase mass conservation, on the other hand, is affected by chemical reaction and is given by

$$
\frac{\partial}{\partial \tilde{t}}\left[(1-\alpha) \tilde{\rho}_{l}\right]+\frac{\partial}{\partial \tilde{x}_{3}}\left[(1-\alpha) \tilde{\rho}_{l} \tilde{u}_{l}\right]=-\tilde{A} \tilde{\rho}_{l}(1-\alpha) \exp \left(-\tilde{E} / \tilde{R}^{\circ} \tilde{T}\right), \quad \tilde{x}_{3}>\tilde{x}_{m},
$$

where $\tilde{E}$ denotes the overall activation energy, $\tilde{R}^{\circ}$ is the universal gas constant, $\tilde{A}$ is the preexponential rate constant and $\tilde{T}$ is the temperature, 
Utilizing Eq. (2.3) to eliminate the reaction-rate expression, we may write overall energy conservation as

$$
\begin{aligned}
\frac{\partial}{\partial \tilde{t}}\left\{\left[\tilde{\rho}_{s} \tilde{c}_{s}\left(1-\alpha_{s}\right)+\right.\right. & \left.\left.\tilde{\rho}_{g} \tilde{c}_{g} \alpha_{s}\right] \tilde{T}\right\}+\frac{\partial}{\partial \tilde{x}_{3}}\left(\tilde{\rho}_{g} \tilde{c}_{g} \tilde{u}_{g} \alpha_{s} \tilde{T}\right) \\
& =\tilde{\nabla} \cdot\left\{\left[\tilde{\lambda}_{s}\left(1-\alpha_{s}\right)+\tilde{\lambda}_{g} \alpha_{s}\right] \tilde{\nabla} \tilde{T}\right\}+\alpha_{s} \frac{\partial \tilde{p}_{g}}{\partial \tilde{t}}, \quad \tilde{x}_{3}<\tilde{x}_{m}
\end{aligned}
$$

and

$$
\begin{aligned}
\frac{\partial}{\partial \tilde{t}}\left[\tilde{\rho}_{l}(1-\alpha)\left(\tilde{Q}+\tilde{c}_{l} \tilde{T}\right)\right. & \left.+\tilde{\rho}_{g} \tilde{c}_{g} \alpha \tilde{T}\right]+\frac{\partial}{\partial \tilde{x}_{3}}\left[\tilde{\rho}_{l} \tilde{u}_{l}(1-\alpha)\left(\tilde{Q}+\tilde{c}_{l} \tilde{T}\right)+\tilde{\rho}_{g} \tilde{c}_{g} \tilde{u}_{g} \alpha \tilde{T}\right] \\
& =\tilde{\nabla} \cdot\left\{\left[\tilde{\lambda}_{l}(1-\alpha)+\tilde{\lambda}_{g} \alpha\right] \tilde{\nabla} \tilde{T}\right\}+\alpha \frac{\partial \tilde{p}_{g}}{\partial \tilde{t}}, \quad \tilde{x}_{3}>\tilde{x}_{m}
\end{aligned}
$$

where $\tilde{c}, \tilde{\lambda}$ and $\tilde{p}$ denote specific heat (at constant pressure for the gas), thermal conductivity and pressure, respectively, the first two of which are assumed constant.

The equation of state for the gas, which is assumed to be ideal, is given by

$$
\tilde{p}_{g}=\tilde{\rho}_{g} \tilde{R}^{\circ} \tilde{T} / \tilde{W}_{g}
$$

where $\tilde{W}_{g}$ is the average molecular weight of the gas, assumed constant. Conservation of momentum, however, is accounted for in a more approximate fashion. In particular (cf. [4,12,16]) condensed-phase momentum is reduced to a kinematic expression for the liquid-phase velocity $u_{l}$ and the previously stated assumption that $u_{s}=0$, while gas-phase momentum is replaced by Darcy's law for the gas-phase velocity $u_{g}$ in the solid/gas region and by the assumption that $p_{g}$ is constant throughout the liquid/gas region. That is,

$$
\bar{u}_{g}=-\frac{\tilde{\kappa}\left(\alpha_{s}\right)}{\alpha_{s} \tilde{\mu}_{g}} \frac{\partial \tilde{p}_{g}}{\partial \tilde{x}_{3}}, \quad x<\tilde{x}_{m} ; \quad u_{l}=-\frac{\partial \tilde{x}_{m}}{\partial t}\left(\frac{\tilde{\rho}_{s}}{\tilde{\rho}_{l}}-1\right), \quad \tilde{p}_{g}=\tilde{p}_{g}^{b}>\tilde{p}_{g}^{u}, \quad \tilde{x}>\tilde{x}_{m},
$$

where $\tilde{\kappa}\left(\alpha_{s}\right)$ is the permeability coefficient of the porous solid and $\tilde{\mu}_{g}$ is the gas-phase viscosity. Equations (2.1) - (2.7) constitute a closed set, which may be solved subject to appropriate boundary and continuity conditions.

The boundary conditions appropriate for the confined deflagration problem described above are expressed as

$$
\begin{aligned}
& \alpha=\alpha_{s} \text { for } \tilde{x}_{3}<\tilde{x}_{m} ; \quad \tilde{u}_{g} \rightarrow 0, \quad \tilde{T} \rightarrow \tilde{T}_{u}, \quad \tilde{p}_{g} \rightarrow \tilde{p}_{g}^{u} ; \text { as } \tilde{x}_{3} \rightarrow-\infty, \\
& \alpha \rightarrow 1 \text { for } \tilde{x}_{3}>\tilde{x}_{m} ; \quad \tilde{p}_{g} \rightarrow \tilde{p}_{g}^{b}, \quad \tilde{u}_{g} \rightarrow \tilde{u}_{g}^{b}, \quad \tilde{T} \rightarrow \tilde{T}_{b} \text { as } \tilde{x}_{3} \rightarrow+\infty,
\end{aligned}
$$

where $\tilde{x}_{3}= \pm \infty$ are interpreted as large with respect to the multiphase flame location. In addition, continuity and jump conditions across the melting surface are specified as

$$
\begin{gathered}
\left.\alpha\right|_{\tilde{x}_{3}=\tilde{x}_{m}}=\alpha_{s},\left.\quad \tilde{T}\right|_{\tilde{x}_{3}=\tilde{x}_{m}}=\tilde{T}_{m},\left.\quad \tilde{p}_{g}\right|_{\tilde{x}_{3}=\tilde{x}_{m}^{-}}=\left.\tilde{p}_{g}\right|_{\tilde{x}_{3}=\tilde{x}_{m}^{+}}=\tilde{p}_{g}^{b}, \\
\left.\tilde{u}_{g}\right|_{\tilde{x}_{3}=\tilde{x}_{m}^{+}}=\left.\tilde{u}_{g}\right|_{\tilde{x}_{3}=\tilde{x}_{m}^{-}}=-\left.\frac{\tilde{\kappa}\left(\alpha_{s}\right)}{\alpha_{s} \tilde{\mu}_{g}} \frac{\partial \tilde{p}_{g}}{\partial \tilde{x}_{3}}\right|_{\tilde{x}_{3}=\tilde{x}_{m}^{-}},
\end{gathered}
$$




$$
\begin{array}{r}
G_{m}\left\{\left.\left[\tilde{\lambda}_{l}\left(1-\alpha_{s}\right)+\tilde{\lambda}_{g} \alpha_{s}\right] \hat{\mathbf{n}}_{m} \cdot \tilde{\nabla} \tilde{T}\right|_{\tilde{x}_{3}=\tilde{x}_{m}^{+}}-\left.\left[\tilde{\lambda}_{s}\left(1-\alpha_{s}\right)+\tilde{\lambda}_{g} \alpha_{s}\right] \hat{\mathbf{n}}_{m} \cdot \tilde{\nabla} \tilde{T}\right|_{\tilde{x}_{3}=\tilde{x}_{m}^{-}}\right\} \\
=\left(1-\alpha_{s}\right)\left\{-\tilde{\rho}_{s} \tilde{\gamma}_{s} \frac{\partial \tilde{x}_{m}}{\partial \tilde{t}}+\left[\tilde{\rho}_{l} \tilde{c}_{l}\left(\left.\tilde{u}_{l}\right|_{\tilde{x}_{3}=\tilde{x}_{m}^{+}}-\frac{\partial \tilde{x}_{m}}{\partial \tilde{t}}\right)-\tilde{\rho}_{s} \tilde{c}_{s}\left(-\frac{\partial \tilde{x}_{m}}{\partial \tilde{t}}\right)\right] \tilde{T}_{m}\right\},
\end{array}
$$

where the unit normal $\hat{\mathbf{n}}_{m}$ has $\tilde{x}_{1^{-}}, \tilde{x}_{2^{-}}$and $\tilde{x}_{3^{-}}$components $-G_{m}^{-1}\left(\partial \tilde{x}_{m} / \partial \tilde{x}_{1}\right),-G_{m}^{-1}\left(\partial \tilde{x}_{m} / \partial \tilde{x}_{2}\right)$ and $G_{m}^{-1}$, respectively, the geometric factor $G_{m}$ being given by

$$
G_{m}=\sqrt{1+\left(\partial \tilde{x}_{m} / \partial \tilde{x}_{1}\right)^{2}+\left(\partial \tilde{x}_{m} / \partial \tilde{x}_{2}\right)^{2}}
$$

We note that $\tilde{\gamma}_{s}$ is the heat of melting of the solid at temperature $\tilde{T}=0$, defined in this work to have positive values when melting is endothermic. The formulation presented here represents a reasonable model for certain classes of energetic materials, as typified by common nitramine propellants such as HMX and RDX.

\section{Nondimensionalizations and the Outer Problem}

Prior to analyzing the present model, it is convenient to introduce nondimensional quantities. In particular, nondimensional variables are defined as

$$
x_{i}=\frac{\tilde{\rho}_{s} \tilde{c}_{s} \tilde{U}}{\tilde{\lambda}_{s}} \tilde{x}_{i}, \quad t=\frac{\tilde{\rho}_{s} \tilde{c}_{s} \tilde{U}^{2}}{\tilde{\lambda}_{s}} \tilde{t}, \quad T=\frac{\tilde{T}}{\tilde{T}_{u}}, \quad u_{l, g}=\frac{\tilde{u}_{l, g}}{\tilde{U}}, \quad \rho_{g}=\frac{\tilde{\rho}_{g}}{\tilde{\rho}_{g}^{u}}, \quad p_{g}=\frac{\tilde{p}_{g}}{\tilde{p}_{g}^{u}},
$$

where $\tilde{\rho}_{g}^{u}=\tilde{p}_{g}^{u} \tilde{W}_{g} / \tilde{R}^{\circ} \tilde{T}_{u}$ is the gas density evaluated at $\tilde{x}_{3}=-\infty$, and the characteristic velocity $\tilde{U}=-d \tilde{x}_{m} / d \tilde{t}$ is the propagation speed for the case of a quasi-steady, planar deflagration. Corresponding nondimensional parameters are given by

$$
\begin{aligned}
& r=\frac{\tilde{\rho}_{l}}{\tilde{\rho}_{s}}, \quad \hat{r}=\frac{\tilde{\rho}_{g}^{u}}{\tilde{\rho}_{s}}, \quad l=\frac{\tilde{\lambda}_{l}}{\tilde{\lambda}_{s}}, \quad \hat{l}=\frac{\tilde{\lambda}_{g}}{\tilde{\lambda}_{s}}, \quad b=\frac{\tilde{c}_{l}}{\tilde{c}_{s}}, \quad \hat{b}=\frac{\tilde{c}_{g}}{\tilde{c}_{s}}, \quad \gamma_{s}=-\frac{\tilde{\gamma}_{s}}{\tilde{c}_{s} \tilde{T}_{u}}, \quad Q=\frac{\tilde{Q}}{\tilde{c}_{s} \tilde{T}_{u}}, \\
& \kappa=\frac{\tilde{\rho}_{s} \tilde{c}_{s} \tilde{p}_{g}^{u} \tilde{\kappa}\left(\alpha_{s}\right)}{\tilde{\lambda}_{s} \tilde{\mu}_{g}}, \quad \hat{\pi}=\frac{\tilde{p}_{g}^{u}}{\tilde{\rho}_{s} \tilde{c}_{s} \tilde{T}_{u}}=\hat{r} \hat{b} \chi, \quad \chi=\frac{\gamma-1}{\gamma}, \quad N=\frac{\tilde{E}}{\tilde{R}^{\circ} \tilde{T}_{b}}, \quad \Lambda=\frac{\tilde{\lambda}_{s} \tilde{A}}{\tilde{\rho}_{s} \tilde{c}_{s} \tilde{U}^{2}} e^{-N},
\end{aligned}
$$

where $\gamma$ is the ratio of specific heats for the gas and $\Lambda$ is an appropriate burning-rate eigenvalue.

As in previous studies, we consider the limit of large activation energy, in which case the reaction zone collapses to a reaction front (on the scale of the overall combustion wave) whose location is defined as $x_{3}=x_{r}\left(x_{1}, x_{2}, t\right)>x_{m}\left(x_{1}, x_{2}, t\right)$. Hence, it is convenient to transform to the moving coordinate system $\left(x_{1}, x_{2}, \zeta\right)$, where

$$
\zeta=x_{3}-x_{r}\left(x_{1}, x_{2}, t\right)
$$

Introducing these nondimensionalizations and transformation into the problem defined in the previous section, we obtain the system of equations

$$
u_{l}=-\frac{\partial x_{m}}{\partial t}\left(\frac{1}{r}-1\right)
$$




$$
\begin{aligned}
& \frac{\partial \rho_{g}}{\partial t}+\frac{\partial}{\partial \zeta}\left[\rho_{g}\left(u_{g}-\frac{\partial x_{r}}{\partial t}\right)\right]=0, \quad \zeta<-\left(x_{r}-x_{m}\right) \\
& \frac{\partial}{\partial t}\left[\alpha\left(\hat{r} \rho_{g}-r\right)\right]+\frac{\partial}{\partial \zeta}\left[r(1-\alpha)\left(u_{l}-\frac{\partial x_{r}}{\partial t}\right)+\hat{r} \alpha \rho_{g}\left(u_{g}-\frac{\partial x_{r}}{\partial t}\right)\right]=0, \quad \zeta>-\left(x_{r}-x_{m}\right), \\
& \frac{\partial \alpha}{\partial t}-\frac{\partial}{\partial \zeta}\left[(1-\alpha)\left(u_{l}-\frac{\partial x_{r}}{\partial t}\right)\right]=\Lambda(1-\alpha) \exp \left[N\left(1-\frac{T_{b}}{T}\right)\right], \quad \zeta>-\left(x_{r}-x_{m}\right), \\
& \frac{\partial}{\partial t}\left\{\left[\left(1-\alpha_{s}\right)+\hat{r} \hat{b} \alpha_{s} \rho_{g}\right] T\right\}+\frac{\partial}{\partial \zeta}\left\{\left[\left(1-\alpha_{s}\right)\left(-\frac{\partial x_{r}}{\partial t}\right)+\hat{r} \hat{b} \alpha_{s}\left(u_{g}-\frac{\partial x_{r}}{\partial t}\right) \rho_{g}\right] T\right\} \\
& =\nabla_{r} \cdot\left\{\left[1-\alpha_{s}+\hat{l} \alpha_{s}\right] \nabla_{r} T\right\}+\hat{\pi} \alpha_{s}\left(\frac{\partial p_{g}}{\partial t}-\frac{\partial x_{r}}{\partial t} \frac{\partial p_{g}}{\partial \zeta}\right), \quad \zeta<-\left(x_{r}-x_{m}\right), \\
& \frac{\partial}{\partial t}\left[r(1-\alpha)(Q+b T)+\hat{r} \hat{b} \alpha \rho_{g} T\right]+\frac{\partial}{\partial \zeta}\left[r(1-\alpha)\left(u_{l}-\frac{\partial x_{r}}{\partial t}\right)(Q+b T)+\hat{r} \hat{b} \alpha\left(u_{g}-\frac{\partial x_{r}}{\partial t}\right) \rho_{g} T\right] \\
& =\nabla_{r} \cdot\left\{[l(1-\alpha)+\hat{l} \alpha] \nabla_{r} T\right\}, \quad \zeta>-\left(x_{r}-x_{m}\right), \\
& \rho_{g} T=p_{g}
\end{aligned}
$$

subject to

$$
\begin{gathered}
\alpha=\alpha_{s}, \quad u_{g}=-\frac{\kappa\left(\alpha_{s}\right)}{\alpha_{s}} \frac{\partial p_{g}}{\partial \zeta} \text { for } \zeta \leq-\left(x_{r}-x_{m}\right) ; \quad p_{g} \rightarrow 1 \quad\left(u_{g} \rightarrow 0\right), \quad T \rightarrow 1 \text { as } \zeta \rightarrow-\infty \\
p_{g}=p_{g}^{b} \text { for } \zeta>-\left(x_{r}-x_{m}\right) ; \quad \alpha \rightarrow 1, \quad T \rightarrow T_{b} \text { as } \zeta \rightarrow+\infty \\
\left.T\right|_{\zeta=-\left(x_{r}-x_{m}\right)}=T_{m}, \\
{\left.\left[l\left(1-\alpha_{s}\right)+\hat{l} \alpha_{s}\right] \hat{\mathbf{n}}_{m} \cdot \nabla_{r} T\right|_{\zeta=-\left(x_{r}-x_{m}\right)^{+}}-\left.\left(1-\alpha_{s}+\hat{l} \alpha_{s}\right) \hat{\mathbf{n}}_{m} \cdot \nabla_{r} T\right|_{\zeta=-\left(x_{r}-x_{m}\right)^{-}}} \\
=-G_{m}^{-1} \frac{\partial x_{m}}{\partial t}\left(1-\alpha_{s}\right)\left[-\gamma_{s}+(b-1) T_{m}\right]
\end{gathered}
$$

with all variables continuous across the melting surface. Here, the operator $\nabla_{r}$ is the nondimensional gradient operator in the moving coordinate system and is given by $\nabla_{r}=\left(\partial / \partial x_{1}-\right.$ $\left.\left\{\partial x_{r} / \partial x_{1}\right\} \partial / \partial \zeta, \partial / \partial x_{2}-\left\{\partial x_{r} / \partial x_{2}\right\} \partial / \partial \zeta, \partial / \partial \zeta\right)$

The above system of equations is similar to the unconfined stability problem considered previously [9], but owing to the overpressure associated with the confined geometry in the present work, there appear additional terms in Eq. (3.8) associated with a spatially varying pressure field in the solid/gas region, and Darcy's law in Eqs. (3.11) appears in place of the constant-pressure assumption that was used in the treatment of the confined case. An analysis of the completely quasi-steady problem was presented in an earlier study [12], resulting in an expression for the burning-rate eigenvalue $\Lambda$ and an expression for the burned temperature $T_{b}$ that contains an explicit dependence on overpressure according to

$$
T_{b}=\frac{\left(1-\alpha_{s}\right)\left(Q+1+\gamma_{s}\right)+\hat{r} \hat{b} \alpha_{s}\left[1+\chi\left(p_{g}^{b}-1\right)\right]}{\hat{b}\left[1+\alpha_{s}(\hat{r}-1)\right]}
$$


It is observed for future reference that $T_{b}$ is an increasing function of the overpressure $p_{g}^{b}-1$ that stems from the preheating (superadiabatic) effect of pressure-driven permeation of hot gases into the unburned material.

To further simplify the stated problem, we assume that the gas phase is completely quasisteady with respect to the instantaneous reaction-front position $\zeta=0$. As a consequence, all time derivatives of gas-phase variables may be neglected in the preceding formulation. The formal justification of such an assumption, which is common in studies of propellant deflagration, is facilitated by introducing additional (short) temporal and spatial scales, associated with the gaseous phase, in the limit of small gas-to-solid density ratio $\hat{r}$. This implies a larger characteristic velocity of the gaseous phase relative to condensed-phase velocities and implies that the gas phase responds on a faster time scale than that associated with condensed-phase processes (cf. $[9,17,18]$ ). Although certain gas-phase dynamics are thus filtered out of the problem (cf. $[19,20]$ ), this approximation does facilitate the study of condensed-phase dynamics while still allowing for an appropriate coupling with gas-phase quantities.

As in previous analyses, we again consider the limit of large activation energy, in which all chemical activity is confined to a thin reaction zone at $\zeta=0$. Solutions in this inner region thus match with those in the outer multiphase preheat region $\zeta<0$ and the outer burned gaseous region $\zeta>0$. In the outer regions, the gas-phase volume fraction and a partial solution for the gas velocity are given by

$$
\begin{gathered}
\alpha= \begin{cases}\alpha_{s}, & \zeta<0 \\
1, & \zeta>0,\end{cases} \\
u_{g}= \begin{cases}-\left(T / p_{g}-1\right) \partial x_{r} / \partial t, & \zeta<-\left(x_{r}-x_{m}\right) \\
-\left(T / p_{g}^{b}-1\right) \partial x_{r} / \partial t, & 0<\zeta<-\left(x_{r}-x_{m}\right) \\
\left(T / p_{g}^{b}\right) g\left(x_{1}, x_{2}, t\right)+\partial x_{r} / \partial t, & \zeta>0,\end{cases}
\end{gathered}
$$

where the latter was obtained from Eqs. (3.5) and (3.6) using Eq. (3.16) and the aforementioned quasi-steady approximation for the gas phase, and the function $g$ is a $\zeta$-independent "constant" of integration. The outer equations for temperature and pressure then become, from Eqs. (3.8) and $(3.9)$,

$$
\begin{gathered}
\left(1-\alpha_{s}\right) \frac{\partial T}{\partial t}+\left(1-\alpha_{s}+\hat{r} \hat{b} \alpha_{s}\right)\left(-\frac{\partial x_{r}}{\partial t}\right) \frac{\partial T}{\partial \zeta}= \\
\left(1-\alpha_{s}+\hat{l} \alpha_{s}\right) \nabla_{r}^{2} T+\hat{r} \hat{b} \chi \alpha_{s}\left(-\frac{\partial x_{r}}{\partial t}\right) \frac{\partial p_{g}}{\partial \zeta}, \quad \zeta<-\left(x_{r}-x_{m}\right), \\
r b\left(1-\alpha_{s}\right) \frac{\partial T}{\partial t}+\left[r b\left(1-\alpha_{s}\right)+\hat{r} \hat{b} \alpha_{s}\right]\left(-\frac{\partial x_{r}}{\partial t}\right) \frac{\partial T}{\partial \zeta}-b(1-r)\left(1-\alpha_{s}\right) \frac{\partial x_{m}}{\partial t} \frac{\partial T}{\partial \zeta} \\
=\left[l\left(1-\alpha_{s}\right)+\hat{l} \alpha_{s}\right] \nabla_{r}^{2} T, \quad-\left(x_{r}-x_{m}\right)<\zeta<0, \\
\hat{r} \hat{b} g \frac{\partial T}{\partial \zeta}=\hat{l} \nabla_{r}^{2} T, \quad \zeta>0,
\end{gathered}
$$


where the use of the first of Eqs. (3.17) in conjunction with Darcy's law in Eqs. (3.11) provides the additional relation

$$
T=p_{g}\left[1+\frac{\kappa}{\alpha_{s}} \frac{\partial p_{g}}{\zeta}\left(\frac{\partial x_{r}}{\partial t}\right)^{-1}\right], \quad \zeta<-\left(x_{r}-x_{m}\right)
$$

Here, the Laplacian operator $\nabla_{r}^{2}$ in the $\left(x_{1}, x_{2}, \zeta\right)$ coordinate system is given by

$$
\nabla_{r}^{2}=\frac{\partial^{2}}{\partial x_{1}^{2}}+\frac{\partial^{2}}{\partial x_{2}^{2}}+G_{r}^{2} \frac{\partial^{2}}{\partial \zeta^{2}}-2 \frac{\partial x_{r}}{\partial x_{1}} \frac{\partial^{2}}{\partial x_{1} \partial \zeta}-2 \frac{\partial x_{r}}{\partial x_{2}} \frac{\partial^{2}}{\partial x_{2} \partial \zeta}-\left(\frac{\partial^{2} x_{r}}{\partial x_{1}^{2}}+\frac{\partial^{2} x_{r}}{\partial x_{2}^{2}}\right) \frac{\partial}{\partial \zeta},
$$

where

$$
G_{r} \equiv \sqrt{1+\left(\partial x_{r} / \partial x_{1}\right)^{2}+\left(\partial x_{r} / \partial x_{2}\right)^{2}}
$$

These solutions are subject to the boundary and interface conditions (3.11) - (3.14) on temperature and pressure, but they are not sufficient for the complete determination of the outer solution. Additional jump and continuity conditions across the thin reaction zone are required, as well as an expression for $g\left(x_{1}, x_{2}, t\right)$ in the last of Eqs. (3.17). By integrating Eq. (3.9) across the thin reaction region and using the results given by Eqs. (3.16) and (3.17), one may obtain two additional continuity/jump relations as

$$
\left.T\right|_{\zeta=0^{-}}=\left.T\right|_{\zeta=0^{+}}
$$

and

$$
\begin{aligned}
\left.\left\{\hat{r} \hat{b} g+\left[\left(1-\alpha_{s}\right) r b+\alpha_{s} \hat{r} \hat{b}\right] \frac{\partial x_{r}}{\partial t}+\left(1-\alpha_{s}\right)(1-r) b \frac{\partial x_{m}}{\partial t}\right\} T\right|_{\zeta=0} & \\
+\left(1-\alpha_{s}\right) Q\left[r \frac{\partial x_{r}}{\partial t}+(1-r) \frac{\partial x_{m}}{\partial t}\right] & -\left.(l-\hat{l})\left(1-\alpha_{s}\right)\left(\frac{\partial x_{r}}{\partial x_{1}} \frac{\partial T}{\partial x_{1}}+\frac{\partial x_{r}}{\partial x_{2}} \frac{\partial T}{\partial x_{2}}\right)\right|_{\zeta=0} \\
& =G_{r}^{2}\left\{\left.\hat{l} \frac{\partial T}{\partial \zeta}\right|_{\zeta=0^{+}}-\left.\left[l\left(1-\alpha_{s}\right)+\hat{l} \alpha_{s}\right] \frac{\partial T}{\partial \zeta}\right|_{\zeta=0^{-}}\right\} .
\end{aligned}
$$

The additional required conditions are then obtained from an analysis of the reaction region and a matching of the solutions obtained therein with the outer solutions. In this context, we assume an expansion of the outer solutions in the form $T \sim T^{(0)}+N^{-1} T^{(1)}+N^{-2} T^{(2)}+\cdots$, and similarly for the function $g$, that are to be matched with corresponding expansions in the reaction zone.

\section{Reaction-Zone Solutions}

Spatial variations in the reaction zone occur on a short length scale relative to that associated with the outer regions. Hence, the stretched normal coordinate $\eta$ is introduced as

$$
\psi=\beta \zeta=\beta\left(x_{3}-x_{r}\right), \quad \beta \equiv\left(1-T_{b}^{-1}\right) N
$$


where $\beta$ is the Zel'dovich number. We also introduce the normalized temperature variable $\Theta=$ $(T-1) /\left(T_{b}-1\right)$ and seek solutions to the inner problem in the form

$$
\begin{aligned}
\alpha & \sim \alpha_{0}+\beta^{-1} \alpha_{1}+\beta^{-2} \alpha_{2}+\cdots, \\
u_{g} & \sim u_{0}+\beta^{-1} u_{1}+\beta^{-2} u_{2}+\cdots, \\
\Theta & \sim 1+\beta^{-1} \theta_{1}+\beta^{-2} \theta_{2}+\cdots, \\
\Lambda & \sim \beta\left(\Lambda_{0}+\beta^{-1} \Lambda_{1}+\beta^{-2} \Lambda_{2}+\cdots\right),
\end{aligned}
$$

where, since $p_{g}=p_{b}^{b}$ in the liquid/gas region, no such expansion for the pressure $p_{g}$ is required. Equation (3.6) at leading order in the inner variables is consequently given by

$$
\left(r-\hat{r} \frac{p_{g}^{b}}{T_{b}}\right) \frac{\partial x_{r}}{\partial t} \frac{\partial \alpha_{0}}{\partial \psi}+(1-r) \frac{\partial x_{m}}{\partial t} \frac{\partial \alpha_{0}}{\partial \psi}+\hat{r} \frac{p_{g}^{b}}{T_{b}} \frac{\partial}{\partial \psi}\left(\alpha_{0} u_{0}\right)=0
$$

which, when integrated and matched with the outer solution (3.17), determines $u_{0}$ and the leadingorder coefficient $g^{(0)}$ as

$$
\begin{aligned}
& u_{0}=\frac{T_{b}\left(\alpha_{0}-\alpha_{s}\right)}{p_{g}^{b} \hat{r} \alpha_{0}}\left[(\hat{r}-r) \frac{\partial x_{r}}{\partial t}-(1-r) \frac{\partial x_{m}}{\partial t}\right]-\left(\frac{T_{b}}{p_{g}^{b}}-1\right) \frac{\partial x_{r}}{\partial t}, \\
& g^{(0)}\left(x_{1}, x_{2}, t\right)=\frac{1-\alpha_{s}}{\hat{r}}\left[(\hat{r}-r) \frac{\partial x_{r}}{\partial t}-(1-r) \frac{\partial x_{m}}{\partial t}\right]-\frac{\partial x_{r}}{\partial t} .
\end{aligned}
$$

The remaining part of the leading-order problem is determined from Eqs. (3.7) and (3.9) as

$$
\begin{gathered}
{\left[r \frac{\partial x_{r}}{\partial t}+(1-r) \frac{\partial x_{m}}{\partial t}\right] \frac{\partial \alpha_{0}}{\partial \psi}=-r \Lambda_{0}\left(1-\alpha_{0}\right) e^{\theta_{1}}} \\
{\left[r \frac{\partial x_{r}}{\partial t}+(1-r) \frac{\partial x_{m}}{\partial t}\right]\left[Q+(b-\hat{b}) T_{b}\right] \frac{\partial \alpha_{0}}{\partial \psi}=\left(T_{b}-1\right) G_{r}^{2} \frac{\partial}{\partial \psi}\left\{\left[l+(\hat{l}-l) \alpha_{0}\right] \frac{\partial \theta_{1}}{\partial \psi}\right\},}
\end{gathered}
$$

subject to the matching conditions

$$
\begin{gathered}
\alpha_{0} \rightarrow 1,\left.\quad \theta_{1} \sim \Theta^{(1)}\right|_{\zeta=0^{+}} \text {as } \psi \rightarrow+\infty, \\
\alpha_{0} \rightarrow \alpha_{s},\left.\quad \theta_{1} \sim \Theta^{(1)}\right|_{\zeta=0^{-}}+\left.\psi \frac{\partial \Theta^{(0)}}{\partial \zeta}\right|_{\zeta=0^{-}} \text {as } \psi \rightarrow-\infty,
\end{gathered}
$$

where the normalized outer temperature coefficients are defined as $\Theta^{(i)}=\left(T^{(i)}-1\right) /\left(T_{b}-1\right)$. We observe that since, according to Eq. (4.1), the spatial variations in the normal direction are large compared to variations with respect to the transverse directions and with respect to time, the reaction-zone problem is, to leading order, always quasi-steady and quasi-planar.

Analysis of Eqs. (4.5) - (4.8) is essentially unchanged from that in the confined problem $[9,12]$, since $p_{g}$, though greater than $p_{g}^{u}$, remains constant in the liquid/gas region. Repeating this 
analysis for the present problem, we find the additional jump conditions across the reaction region to be given, in terms of the original outer variable $T$, by

$$
-r \frac{\partial x_{r}}{\partial t}-(1-r) \frac{\partial x_{m}}{\partial t}=\left[1+\left(\frac{\partial x_{r}}{\partial x_{1}}\right)^{2}+\left(\frac{\partial x_{r}}{\partial x_{2}}\right)^{2}\right]^{1 / 2} \exp \left(-\frac{\beta}{2} \cdot \frac{T_{b}-\left.T\right|_{\zeta=0}}{T_{b}-1}\right)
$$

and

$$
\begin{gathered}
\left.\hat{l} \frac{\partial T}{\partial \zeta}\right|_{\zeta=0^{+}}-\left.\left[l+(\hat{l}-l) \alpha_{s}\right] \frac{\partial T}{\partial \zeta}\right|_{\zeta=0^{-}}+\left.\left(1-\alpha_{s}\right)(l-\hat{l}) G_{r}^{-2}\left(\frac{\partial x_{r}}{\partial x_{1}} \frac{\partial T}{\partial x_{1}}+\frac{\partial x_{r}}{\partial x_{2}} \frac{\partial T}{\partial x_{2}}\right)\right|_{\zeta=0} \\
=-\left(1-\alpha_{s}\right)\left[Q+\left.(b-\hat{b}) T\right|_{\zeta=0}\right] G_{r}^{-1} \exp \left(-\frac{\beta}{2} \cdot \frac{T_{b}-\left.T\right|_{\zeta=0}}{T_{b}-1}\right)
\end{gathered}
$$

where the outer expansions have been truncated so as to produce a closed set of conditions (cf. $[9]$ ).

\section{Summary of the Asymptotic Model and its Basic Solution}

The analysis presented in the previous sections thus reduces the thin reaction region to a reaction sheet described by a set of jump and continuity conditions, eliminating the need for further consideration of its structure. After collecting these results, the asymptotic model, valid in the limit of large activation energy, for the outer solution is given by

$$
\begin{aligned}
& \left(1-\alpha_{s}\right)\left\{\begin{array}{c}
1 \\
r b \\
0
\end{array}\right\} \frac{\partial T}{\partial t}-\left\{\begin{array}{c}
1+\alpha_{s}(\hat{r} \hat{b}-1) \\
r b+\alpha_{s}(\hat{r} \hat{b}-r b) \\
\hat{r} \hat{b}
\end{array}\right\} \frac{\partial x_{r}}{\partial t} \frac{\partial T}{\partial \zeta} \\
& -\left(1-\alpha_{s}\right)\left[(1-r)\left\{\begin{array}{l}
0 \\
b \\
\hat{b}
\end{array}\right\} \frac{\partial x_{m}}{\partial t}+(r-\hat{r})\left\{\begin{array}{l}
0 \\
0 \\
\hat{b}
\end{array}\right\} \frac{\partial x_{r}}{\partial t}\right] \frac{\partial T}{\partial \zeta} \\
& =\left\{\begin{array}{c}
1+\alpha_{s}(\hat{l}-1) \\
l+\alpha_{s}(\hat{l}-l) \\
\hat{l}
\end{array}\right\} \nabla_{r}^{2} T-\hat{r} \hat{b} \chi \alpha_{s}\left\{\begin{array}{l}
1 \\
0 \\
0
\end{array}\right\} \frac{\partial x_{r}}{\partial t} \frac{\partial p_{g}}{\partial \zeta}, \begin{array}{c}
\zeta<-\left(x_{r}-x_{m}\right) \\
-\left(x_{r}-x_{m}\right)<\zeta<0, \\
\zeta>0
\end{array} \\
& T=p_{g}\left[1+\frac{\kappa}{\alpha_{s}} \frac{\partial p_{g}}{\partial \zeta}\left(\frac{\partial x_{r}}{\partial t}\right)^{-1}\right], \quad \zeta<-\left(x_{r}-x_{m}\right) \\
& -r \frac{\partial x_{r}}{\partial t}-(1-r) \frac{\partial x_{m}}{\partial t}=G_{r} \exp \left(-\frac{\beta}{2} \cdot \frac{T_{b}-\left.T\right|_{\zeta=0}}{T_{b}-1}\right)
\end{aligned}
$$

subject to the boundary and jump conditions

$$
\begin{array}{r}
T \rightarrow p_{g} \rightarrow 1 \text { as } \zeta \rightarrow-\infty, \quad T \rightarrow T_{b} \text { as } \zeta \rightarrow+\infty, \\
T=T_{m} \text { at } \zeta=-\left(x_{r}-x_{m}\right), \quad p_{g}=p_{g}^{b} \text { for } \zeta \geq-\left(x_{r}-x_{m}\right),
\end{array}
$$




$$
\begin{gathered}
\left(-\frac{\partial x_{m}}{\partial x_{1}},-\frac{\partial x_{m}}{\partial x_{2}}, 1\right) \cdot\left\{\left.\left[l+\alpha_{s}(\hat{l}-l)\right] \nabla_{r} T\right|_{\zeta=-\left(x_{r}-x_{m}\right)^{+}}-\left.\left[1+\alpha_{s}(\hat{l}-1)\right] \nabla_{r} T\right|_{\zeta=-\left(x_{r}-x_{m}\right)-}\right\} \\
=-\frac{\partial x_{m}}{\partial t}\left(1-\alpha_{s}\right)\left[-\gamma_{s}+(b-1) T_{m}\right] \\
\left.T\right|_{\zeta=0^{-}}=\left.T\right|_{\zeta=0^{+}} \\
\left.\hat{l} \frac{\partial T}{\partial \zeta}\right|_{\zeta=0^{+}}-\left.\left[l+(\hat{l}-l) \alpha_{s}\right] \frac{\partial T}{\partial \zeta}\right|_{\zeta=0^{-}}+\left.\left(1-\alpha_{s}\right)(l-\hat{l}) G_{r}^{-2}\left(\frac{\partial x_{r}}{\partial x_{1}} \frac{\partial T}{\partial x_{1}}+\frac{\partial x_{r}}{\partial x_{2}} \frac{\partial T}{\partial x_{2}}\right)\right|_{\zeta=0} \\
=-\left(1-\alpha_{s}\right)\left[Q+\left.(b-\hat{b}) T\right|_{\zeta=0}\right] G_{r}^{-1} \exp \left(-\frac{\beta}{2} \cdot \frac{T_{b}-\left.T\right|_{\zeta=0}}{T_{b}-1}\right)
\end{gathered}
$$

where the operator $\nabla_{r}$ was given below Eq. (3.14), and expressions for $\nabla_{r}^{2}=\nabla_{r} \cdot \nabla_{r}$ and $G_{r}$ were given by Eqs. (3.22) and (3.23), respectively. We observe that the jump condition (5.7) across the reaction zone as well as the propagation law (5.3) are both sensitive to local temperature perturbations, the exponential form of which is induced by the Arrhenius nature of the reaction rate in the limit of large, but finite, activation energy.

Equations $(5.1)-(5.7)$ constitute a sufficiently closed boundary-value problem for $T, p_{g}, x_{r}$ and $x_{m}$, where a scalar equation for $p_{g}$ is obtained from a combination of Eqs. (5.1) and (5.2) in the region $\zeta<-\left(x_{r}-x_{m}\right)$, and the remaining quantities of interest, namely $\alpha_{s}$ and $u_{g}$, were given by Eqs. (3.16) and (3.17). This may be solved subject to appropriate initial conditions, but in the present work we are concerned only with a basic long-time solution and its stability. Consequently, we first describe the basic solution corresponding to a (quasi-)steadily propagating, planar deflagration. Denoting this solution with a zero superscript, it is given in part by

$$
\begin{gathered}
x_{m}^{0}=-t, \quad x_{r}^{0}=x_{m}^{0}+\frac{l\left(1-\alpha_{s}\right)+\hat{l} \alpha_{s}}{b\left(1-\alpha_{s}\right)+\hat{r} \hat{b} \alpha_{s}} \ln \left(\frac{T_{b}-B}{T_{m}-B}\right), \\
T^{0}(\zeta)=\left\{\begin{array}{lr}
B+\left(T_{m}-B\right) \exp \left[\frac{b\left(1-\alpha_{s}\right)+\hat{r} \hat{b} \alpha_{s}}{l\left(1-\alpha_{s}\right)+\hat{l} \alpha_{s}}\left(\zeta+x_{r}^{0}-x_{m}^{0}\right)\right], \quad-\left(x_{r}^{0}-x_{m}^{0}\right)<\zeta<0 \\
T_{b}=\frac{\left(1-\alpha_{s}\right)\left(Q+1+\gamma_{s}\right)+\hat{r} \hat{b} \alpha_{s}\left[1+\chi\left(p_{g}^{b}-1\right)\right]}{\hat{b}\left[1+\alpha_{s}(\hat{r}-1)\right]}, & \zeta>0,
\end{array}\right.
\end{gathered}
$$

with

$$
B \equiv \frac{\left(1-\alpha_{s}\right)\left(1+\gamma_{s}\right)+\hat{r} \hat{b} \alpha_{s}\left[1+\chi\left(p_{g}^{b}-1\right)\right]}{b\left(1-\alpha_{s}\right)+\hat{r} \hat{b} \alpha_{s}}
$$

Expressions for the basic temperature and pressure in the solid/gas region $\zeta<-\left(x_{r}^{0}-x_{m}^{0}\right)$ are determined from the steady, planar version of Eqs. (5.1) and Eq. (5.2). The latter gives $T^{0}$ in terms of $p_{g}^{0}$ as

$$
T^{0}=p_{g}^{0}-\frac{\kappa}{\alpha_{s}} p_{g}^{0} \frac{d p_{g}^{0}}{d \zeta}, \quad \zeta<-\left(x_{r}^{0}-x_{m}^{0}\right)
$$


and a scalar equation for $p_{g}^{0}$ is then obtained by substituting this result into Eq. (5.1) and integrating once to give

$$
\left(1-\alpha_{s}+\hat{r} \hat{b} \alpha_{s}\right)\left[p_{g}^{0}\left(1-\frac{\kappa}{\alpha_{s}} \frac{d p_{g}^{0}}{d \zeta}\right)-1\right]=\left(1-\alpha_{s}+\hat{l} \alpha_{s}\right) \frac{d}{d \zeta}\left[p_{g}^{0}\left(1-\frac{\kappa}{\alpha_{s}} \frac{d p_{g}^{0}}{d \zeta}\right)\right]+\hat{r} \hat{b} \alpha_{s} \chi\left(p_{g}^{0}-1\right)
$$

Finally, from Eq. (3.17) and the last of Eqs. (4.4), the solution for the gas velocity $u_{g}^{0}$ is given in terms of $T^{0}$ and $p_{g}^{0}$ by

$$
u_{g}^{0}= \begin{cases}T^{0} / p_{g}^{0}-1, & \zeta<-\left(x_{r}^{0}-x_{m}^{0}\right) \\ T^{0} / p_{g}^{b}-1, & 0<\zeta<-\left(x_{r}^{0}-x_{m}^{0}\right) \\ \hat{r}^{-1}\left[(1-\hat{r})\left(1-\alpha_{s}\right)+\left(T_{b} / p_{g}^{b}-1\right)\left(1-\alpha_{s}+\alpha_{s} \hat{r}\right)\right], & \zeta>0 .\end{cases}
$$

Although an analytical solution of Eq. (5.11b) for $p_{g}^{0}$ is not apparent, an approximate solution can be obtained by exploiting the realistic smallness of several parameters. In particular, the permeability $\kappa$ may reasonably assumed to be $O(\epsilon)$, where $\beta^{-1} \ll \epsilon \ll 1$ will be formally introduced in Section 7. The details of this construction are outlined in Appendix A and will be introduced as needed in the stability analysis that follows. The essential result, however, is that the pressure profile in the solid/gas region has a boundary-layer structure that induces a corresponding boundary-layer structure in the linear stability problem. That is, for $\mathrm{O}(1)$ values of the overpressure $p_{g}^{b}-1$, there is a relatively thin gas-permeation layer adjacent to the melting surface $\zeta=-\left(x_{r}^{0}-x_{m}^{0}\right)$ where $p_{g}^{0}$ varies from $p_{g}^{b}$ at this surface to near-unity values as the appropriate boundary-layer coordinate becomes large and negative. Inner and outer solutions are thus constructed in a standard fashion, which can then be matched to form a valid composite solution for the solid/gas region. Consequently, there are now four regions to consider: the burned region $\zeta>0$, the liquid/gas preheat zone $-\left(x_{r}^{0}-x_{m}^{0}\right)<\zeta<0$, the thin gas-permeation layer within the solid/gas region, and the outer solid/gas preheat zone $\zeta<-\left(x_{r}^{0}-x_{m}^{0}\right)$. The basic solution given by Eqs. (5.8) - (5.12), obtained from the aforementioned asymptotic construction in the solid/gas region, is illustrated in Figure $1 \mathrm{~b}$.

The important features of this basic solution were discussed previously [12]. In particular, we note for future reference that the burned temperature increases with increased overpressure according to Eq. (3.15), which also results in a significant increase in the burning rate. The increase in $T_{b}$ arises from the pressure-driven permeation of the hot gases into the unburned material, and the resulting significant increase in the burning rate that arises from this convective preheating is associated with the onset of a well-known and much-discussed mode of burning in which convection plays an important role (cf. $[6,12])$. It will be shown later that the value of the burned temperature is also a significant factor in determining the stability of the basic solution and the corresponding neutral stability boundaries. Although the stability analysis described below is in the spirit of previous analyses of the unconfined problem $[8,9]$, the presence of confinement and the consequent 
adoption of Darcy's law in the solid/gas region give rise to the presence of the gas-permeation layer described above that was not present in the unconfined problem. Thus, in order to handle this additional complication, it is necessary to introduce a formal expansion of the stability problem (and the basic solution) in the small parameter $\epsilon$ associated with the thickness of this layer. In contrast, such a formal expansion was not needed in the previous unconfined studies, and the simplifications arising from the assumed smallness of certain parameters were introduced after first deriving the dispersion relation describing the growth or decay of perturbations about the basic solution. The present work thus is specifically focused on determining the effects of confinement, in conjunction with those associated with porosity and two-phase flow, on the analysis of stability of the basic solution.

\section{The Linear Stability Problem}

The linear stability analysis initially follows the pattern described in previous studies [8,9]. First, we introduce the perturbation variables

$$
x_{m}=x_{m}^{0}+\phi_{m}, \quad x_{r}=x_{r}^{0}+\phi_{r}, \quad T=T^{0}(\zeta)+\tau+\phi_{r} \frac{d T^{0}}{d \zeta}, \quad p_{g}=p_{g}^{0}(\zeta)+\mu+\phi_{r} \frac{d p_{g}^{0}}{d \zeta}
$$

where $\mu, \tau, \phi_{r}$ and $\phi_{m}$ denote perturbations in pressure, temperature and the location of the reaction and melting surfaces, respectively. Substituting the above definitions into the system of equations $(5.1)-(5.7)$ and linearizing the equations with respect to the perturbation variables, a linear problem for $\mu, \tau, \phi_{r}$ and $\phi_{m}$ is obtained as

$$
\begin{aligned}
\left(1-\alpha_{s}\right) \frac{\partial \tau}{\partial t}+\left[1+\alpha_{s}(\hat{r} \hat{b}-1)\right] \frac{\partial \tau}{\partial \zeta}=\left[1+\alpha_{s}(\hat{l}-1)\right]\left(\frac{\partial^{2} \tau}{\partial x_{1}^{2}}+\frac{\partial^{2} \tau}{\partial x_{2}^{2}}+\frac{\partial^{2} \tau}{\partial \zeta^{2}}\right) \\
+\hat{r} \hat{b} \chi \alpha_{s} \frac{\partial \mu}{\partial \zeta}+\hat{r} \hat{b} \alpha_{s} \frac{\partial \phi_{r}}{\partial t} \frac{d T^{0}}{d \zeta}-\hat{r} \hat{b} \chi \alpha_{s} \frac{\partial \phi_{r}}{\partial t} \frac{d p_{g}^{0}}{d \zeta}, \quad \zeta<-\left(x_{r}^{0}-x_{m}^{0}\right) \\
\frac{\kappa}{\alpha_{s}} p_{g}^{0} \frac{\partial \mu}{\partial \zeta}+\frac{\kappa}{\alpha_{s}} \frac{d p_{g}^{0}}{d \zeta} \mu+\frac{\partial \phi_{r}}{\partial t}\left(p_{g}^{0}-T^{0}\right)-\mu+\tau=0, \quad \zeta<-\left(x_{r}^{0}-x_{m}^{0}\right)
\end{aligned}
$$

where $p_{g}^{0}-T^{0}=\left(\kappa / \alpha_{s}\right) p_{g}^{0}\left(d p_{g}^{0} / d \zeta\right)$,

$$
\begin{gathered}
r b\left(1-\alpha_{s}\right) \frac{\partial \tau}{\partial t}+\left[b+\alpha_{s}(\hat{r} \hat{b}-b)\right] \frac{\partial \tau}{\partial \zeta}-b(1-r)\left(1-\alpha_{s}\right) \frac{\partial \phi_{m}}{\partial t} \frac{d T^{0}}{d \zeta} \\
=\left[l+\alpha_{s}(\hat{l}-l)\right]\left(\frac{\partial^{2} \tau}{\partial x_{1}^{2}}+\frac{\partial^{2} \tau}{\partial x_{2}^{2}}+\frac{\partial^{2} \tau}{\partial \zeta^{2}}\right)+\hat{r} \hat{b} \alpha_{s} \frac{\partial \phi_{r}}{\partial t} \frac{d T^{0}}{d \zeta}, \quad-\left(x_{r}^{0}-x_{m}^{0}\right)<\zeta<0 \\
\hat{b}\left[1+\alpha_{s}(\hat{r}-1)\right] \frac{\partial \tau}{\partial \zeta}=\hat{l}\left(\frac{\partial^{2} \tau}{\partial x_{1}^{2}}+\frac{\partial^{2} \tau}{\partial x_{2}^{2}}+\frac{\partial^{2} \tau}{\partial \zeta^{2}}\right), \quad \zeta>0 \\
-r \frac{\partial \phi_{r}}{\partial t}-(1-r) \frac{\partial \phi_{m}}{\partial t}=\left.\frac{\beta}{2\left(T_{b}-1\right)} \tau\right|_{\zeta=0^{+}}
\end{gathered}
$$


subject to

$$
\begin{gathered}
\tau \rightarrow 0 \text { as } \zeta \rightarrow \pm \infty, \quad \mu \rightarrow 0 \text { as } \zeta \rightarrow-\infty \\
\left.\left(\tau+\phi_{m} \frac{d T^{0}}{d \zeta}\right)\right|_{\zeta=-\left(x_{r}^{0}-x_{m}^{0}\right)^{-}}=\left.\left(\tau+\phi_{m} \frac{d T^{0}}{d \zeta}\right)\right|_{\zeta=-\left(x_{r}^{0}-x_{m}^{0}\right)^{+}}=\left.\left(\mu+\phi_{m} \frac{d p_{g}^{0}}{d \zeta}\right)\right|_{\zeta=-\left(x_{r}^{0}-x_{m}^{0}\right)^{-}}=0 \\
{\left.\left[l+\alpha_{s}(\hat{l}-l)\right]\left(\frac{\partial \tau}{\partial \zeta}+\phi_{m} \frac{d^{2} T^{0}}{d \zeta^{2}}\right)\right|_{\zeta=-\left(x_{r}^{0}-x_{m}^{0}\right)^{+}}-\left.\left[1+\alpha_{s}(\hat{l}-1)\right]\left(\frac{\partial \tau}{\partial \zeta}+\phi_{m} \frac{d^{2} T^{0}}{d \zeta^{2}}\right)\right|_{\zeta=-\left(x_{r}^{0}-x_{m}^{0}\right)^{-}}} \\
=-\frac{\partial \phi_{m}}{\partial t}\left(1-\alpha_{s}\right)\left[-\gamma_{s}+(b-1) T_{m}\right] \\
\left.\tau\right|_{\zeta=0^{+}}=\left.\left(\tau+\phi_{r} \frac{d T^{0}}{d \zeta}\right)\right|_{\zeta=0^{-}}, \\
\left.\hat{l} \frac{\partial \tau}{\partial \zeta}\right|_{\zeta=0^{+}},\left.\left[l+\alpha_{s}(\hat{l}-l)\right]\left(\frac{\partial \tau}{\partial \zeta}+\phi_{r} \frac{d^{2} T^{0}}{d \zeta^{2}}\right)\right|_{\zeta=0-} \\
=-\frac{1}{2}\left(1-\alpha_{s}\right)\left[\frac{\beta Q}{T_{b}-1}+(b-\hat{b})\left(2+\frac{\beta T_{b}}{T_{b}-1}\right)\right]_{\zeta=0^{+}}
\end{gathered}
$$

Steady, planar burning clearly corresponds to the trivial solution $\phi_{m}=\phi_{r}=\tau=\mu=0$, and nontrivial solutions to the linear stability problem are sought in the general harmonic form

$$
\left\{\begin{array}{c}
\phi_{m} \\
\phi_{r} \\
\tau \\
\mu
\end{array}\right\}=e^{i\left(\omega t \pm k_{1} x_{1} \pm k_{2} x_{2}\right)}\left\{\begin{array}{c}
c_{m} \\
1 \\
\sigma(\zeta) \\
\nu(\zeta)
\end{array}\right\},
$$

where the solution has been normalized by setting the coefficient of $\phi_{r}$ equal to unity. Substituting Eq. (6.12) into the linear stability problem $(6.1)-(6.11)$, the coefficients $\sigma(\zeta), \nu(\zeta), c_{m}$ and $i \omega$ are governed by

$$
\begin{gathered}
\left(1-\alpha_{s}\right) i \omega \sigma+\left[1+\alpha_{s}(\hat{r} \hat{b}-1)\right] \frac{d \sigma}{d \zeta}=\left[1+\alpha_{s}(\hat{l}-1)\right]\left(\frac{d^{2} \sigma}{d \zeta^{2}}-k^{2} \sigma\right) \\
+\hat{r} \hat{b} \chi \alpha_{s} \frac{d \nu}{d \zeta}+\hat{r} \hat{b} \alpha_{s} i \omega \frac{d T^{0}}{d \zeta}-\chi \hat{r} \hat{b} \alpha_{s} i \omega \frac{d p_{g}^{0}}{d \zeta}, \quad \zeta<-\left(x_{r}^{0}-x_{m}^{0}\right), \\
\frac{\kappa}{\alpha_{s}}\left(p_{g}^{0} \frac{d \nu}{d \zeta}+\frac{d p_{g}^{0}}{d \zeta} \nu+i \omega p_{g}^{0} \frac{d p_{g}^{0}}{d \zeta}\right)-\nu+\sigma=0, \quad \zeta<-\left(x_{r}^{0}-x_{m}^{0}\right), \\
r b\left(1-\alpha_{s}\right) i \omega \sigma+\left[b+\alpha_{s}(\hat{r} \hat{b}-b)\right] \frac{d \sigma}{d \zeta}-b(1-r)\left(1-\alpha_{s}\right) i \omega c_{m} \frac{d T^{0}}{d \zeta} \\
=\quad \hat{b}\left[1+\alpha_{s}(\hat{r}-1)\right]\left(\frac{d^{2} \sigma}{d \zeta^{2}}-k^{2} \sigma\right)+\hat{r} \hat{b} \alpha_{s} i \omega \frac{d T^{0}}{d \zeta}, \quad-\left(x_{r}^{0}-x_{m}^{0}\right)<\zeta<0 \\
\hat{b}\left[\frac{d^{2} \sigma}{d \zeta^{2}}-k^{2} \sigma\right), \quad \zeta>0
\end{gathered}
$$


subject to

$$
\begin{aligned}
& \sigma \rightarrow 0 \text { as } \zeta \rightarrow \pm \infty, \quad \nu \rightarrow 0 \text { as } \zeta \rightarrow-\infty \\
& -i \omega r-i \omega(1-r) c_{m}=\left.\frac{\beta}{2\left(T_{b}-1\right)} \sigma\right|_{\zeta=0^{+}} \\
& \left.\left(\sigma+c_{m} \frac{d T^{0}}{d \zeta}\right)\right|_{\zeta=-\left(x_{r}^{0}-x_{m}^{0}\right)^{-}}=\left.\left(\sigma+c_{m} \frac{d T^{0}}{d \zeta}\right)\right|_{\zeta=-\left(x_{r}^{0}-x_{m}^{0}\right)^{+}}=\left.\left(\nu+c_{m} \frac{d p_{g}^{0}}{d \zeta}\right)\right|_{\zeta=-\left(x_{r}^{0}-x_{m}^{0}\right)^{-}}=0 \\
& {\left.\left[l+\alpha_{s}(\hat{l}-l)\right]\left(\frac{d \sigma}{d \zeta}+c_{m} \frac{d^{2} T^{0}}{d \zeta^{2}}\right)\right|_{\zeta=-\left(x_{r}^{0}-x_{m}^{o}\right)^{+}}-\left.\left[1+\alpha_{s}(\hat{l}-1)\right]\left(\frac{d \sigma}{d \zeta}+c_{m} \frac{d^{2} T^{0}}{d \zeta^{2}}\right)\right|_{\zeta=-\left(x_{r}^{0}-x_{m}^{0}\right)^{-}}} \\
& =-i \omega c_{m}\left(1-\alpha_{s}\right)\left[-\gamma_{s}+(b-1) T_{m}\right], \\
& \left.\sigma\right|_{\zeta=0^{+}}=\left.\left(\sigma+\frac{d T^{0}}{d \zeta}\right)\right|_{\zeta=0^{-}} \\
& \left.\hat{l} \frac{d \sigma}{d \zeta}\right|_{\zeta=0^{+}}-\left.\left[l+\alpha_{s}(\hat{l}-l)\right]\left(\frac{d \sigma}{d \zeta}+\frac{d^{2} T^{0}}{d \zeta^{2}}\right)\right|_{\zeta=0^{-}} \\
& =-\left.\frac{1}{2}\left(1-\alpha_{s}\right)\left[\frac{\beta Q}{T_{b}-1}+(b-\hat{b})\left(2+\frac{\beta T_{b}}{T_{b}-1}\right)\right] \sigma\right|_{\zeta=0^{+}},
\end{aligned}
$$

where $k^{2}=k_{1}^{2}+k_{2}^{2}$.

It is readily seen that these equations, though linear, have some non-constant coefficients in the solid-gas region [cf. Eq. (6.14)] that preclude a direct analytical solution. Indeed, these coefficients, which depend on $p_{g}^{0}$, are themselves known only approximately as discussed in Section 5. However, by considering the same reasonable parameter regime that allowed the construction of $p_{g}^{0}$ (Appendix A), approximate analytical solutions in the form of perturbation expansions may be constructed for Eqs. (6.13) - (6.22) as well. In particular, the dispersion relation itself may be determined as a perturbation expansion, yielding a leading-order neutral stability boundary and a sequence of higher-order corrections. The latter reflect the presence of confinement and associated two-phase-flow processes and hence determine their effect on the stability of the basic solution.

\section{Asymptotic Expansion of the Linear Stability Problem}

We continue our analysis by employing a bookkeeping parameter $\epsilon \sim \mathrm{O}(\hat{r}) \ll 1$ and considering a realistic parameter regime in which $\hat{r}, \hat{l}, \kappa$ and $\gamma_{s}$ are all $O(\epsilon)$. The reasonable assumption of weak permeability is actually a generalization of that introduced in the previous analysis [12] of the basic solution, where it was assumed that $\alpha_{s} \sim \mathrm{O}(\epsilon)$ and $\kappa \sim \alpha_{s}^{2} \sim \mathrm{O}\left(\epsilon^{2}\right)$. However, since it is the smallness of the ratio $\kappa / \alpha_{s}$ that results in the simplified boundary-layer structure in the solid/gas region, we may allow the porosity of the solid to be $O(1)$ and its permeability to be $O(\epsilon)$ and still preserve the thin gas-permeation layer in the unburned solid/gas region. The remaining 
scalings, which were also introduced in the analysis of the unconfined problem $[8,9]$ are not critical, but do serve to simplify the analysis of the dispersion relation. For convenience and consistency, further consideration of the problem is thus formally restricted to this parameter regime.

Having introduced a small parameter $\epsilon$ into the linear stability problem, we seek an approximate solution by expanding all quantities, including the basic solution, in appropriate powers of $\epsilon$, both in the inner gas-permeation region and in the outer preheat regions exterior to this thin layer. Appropriate matching conditions for this singular perturbation problem are then applied to completely determine the solutions and the dispersion relation. Therefore, we introduce the scaled parameters $\hat{r}^{*}, \hat{l}^{*}, \kappa^{*}$ and $\gamma_{s}^{*}$ as

$$
\hat{r}=\hat{r}^{*} \epsilon, \quad \hat{l}=\hat{l}^{*} \epsilon, \quad \kappa=\kappa^{*} \epsilon, \quad \gamma_{s}=\gamma_{s}^{*} \epsilon,
$$

and seek a solution to the complex growth rate $i \omega$ in the expanded form

$$
i \omega=i \omega_{0}+i \omega_{1} \epsilon+i \omega_{2} \epsilon^{2}+\cdots
$$

Consequently, all $\epsilon$-dependent quantities, including the basic solution, may be expanded in $\epsilon$ according to

$$
\begin{gathered}
T_{b} \sim T_{b}^{0}+T_{b}^{(1)} \epsilon+T_{b}^{(2)} \epsilon^{2}+\cdots, \quad \beta \sim \beta_{0}+\beta_{1} \epsilon+\beta_{2} \epsilon^{2}+\cdots \\
\left(x_{r}^{0}-x_{m}^{0}\right) \sim\left(x_{r}^{0}-x_{m}^{0}\right)_{0}+\left(x_{r}^{0}-x_{m}^{0}\right)_{1} \epsilon+\left(x_{r}^{0}-x_{m}^{0}\right)_{2} \epsilon^{2}+\cdots, \quad c_{m}=c_{m}^{(0)}+c_{m}^{(1)} \epsilon+c_{m}^{(2)} \epsilon^{2}+\cdots \\
T^{0} \sim T_{0}+T_{1} \epsilon+T_{2} \epsilon^{2}+\cdots, \quad p_{g}^{0}-1 \sim q_{0}+q_{1} \epsilon+q_{2} \epsilon^{2}+\cdots \\
\sigma \sim \sigma^{(0)}+\sigma^{(1)} \epsilon+\sigma^{(2)} \epsilon^{2}+\cdots, \quad \nu \sim \nu^{(-1)} \epsilon^{-1}+\nu^{(0)}+\nu^{(1)} \epsilon+\cdots
\end{gathered}
$$

where the expansions $(7.1 \mathrm{e}, \mathrm{f})$ in the solid/gas region are the outer expansions valid outside the thin gas-permeation layer adjacent to the melting surface, as opposed to the inner expansions that will be introduced shortly. Also, since conditions at the solid/liquid interface will generally be expanded about the leading-order approximation for $\zeta=-\left(x_{r}^{0}-x_{m}^{0}\right)$, we find it useful to introduce the notation $\left(x_{r}^{0}-x_{m}^{0}\right)_{i}$ to denote the quantity $\left(x_{r}^{0}\right)_{i}-\left(x_{m}^{0}\right)_{i}$, where $\left(x_{r, m}^{0}\right)_{i}$ are the coefficients of the $\mathrm{O}\left(\epsilon^{i}\right)$ terms in the $\epsilon$-expansion of $x_{r, m}^{0}$. Finally, in the expansion of $T_{b}^{0}$, we take the overall heat release with respect to the solid $Q_{0}=Q+\gamma_{s}$ to be a fixed parameter.

For $O(1)$ overpressures $p_{g}^{b}-1$ and $O(\epsilon)$ permeabilities, the solid/gas region has a boundarylayer structure as described in Appendix A for the basic solution. To describe the solution in this region, which is of $O(\epsilon)$ in width relative to the outer portion of the solid/gas region, we introduce a stretched coordinate $\hat{\eta}$ defined as

$$
\hat{\eta}=\epsilon^{-1}\left[\zeta+\left(x_{r}^{0}-x_{m}^{0}\right)\right]
$$

and seek solutions in this region as

$$
\sigma \sim \sigma_{b l}^{(0)}+\sigma_{b l}^{(1)} \epsilon+\sigma_{b l}^{(2)} \epsilon^{2}+\cdots, \quad \nu \sim \nu_{b l}^{(-1)} \epsilon^{-1}+\nu_{b l}^{(0)}+\nu_{b l}^{(1)} \epsilon+\cdots,
$$


with the basic solution expanded in this layer as well according to

$$
p_{g}^{0}-1 \sim p_{0}+p_{1} \epsilon+p_{2} \epsilon^{2}+\cdots, \quad T^{0} \sim \hat{T}_{0}+\hat{T}_{1} \epsilon+\hat{T}_{2} \epsilon^{2}+\cdots .
$$

We observe that the perturbation pressure and temperature in Eqs. (7.3) are not of the same order of magnitude (with respect to $\epsilon$ ), which follows from the fact (see below) that only the pressure varies significantly in the boundary layer.

\section{Leading-Order Analysis and Dispersion Relation}

Substituting the above expansions into the stability problem $(6.13)-(6.22)$, we obtain a sequence of problems for the recursive determination of the coefficients in Eqs. (7.1b,d-f) and (7.3), where the ultimate goal is the determination of the set of dispersion relations $i \omega_{0}(k), i \omega_{1}(k), \ldots$ We can now proceed to solve this sequence of perturbation problems in each of the solid/gas, liquid/gas and burned-gas regions. However, as indicated in the previous section, there is a boundary layer adjacent to the melting surface in the first of these. Hence, the problem in the solid/gas region will consist of both inner (boundary-layer) and outer (external) subproblems.

In the outer zone of the solid/gas region, the leading-order version of Eq. (6.13) gives

$$
\left(1-\alpha_{s}\right) i \omega_{0} \sigma^{(0)}+\left(1-\alpha_{s}\right) \frac{d \sigma^{(0)}}{d \zeta}=\left(1-\alpha_{s}\right)\left(\frac{d^{2} \sigma^{(0)}}{d \zeta^{2}}-k^{2} \sigma^{(0)}\right)+\hat{r}^{*} \hat{b} \chi \alpha_{s} \frac{d \nu^{(-1)}}{d \zeta}, \quad \zeta<-\left(x_{r}^{0}-x_{m}^{0}\right)_{0}
$$

where the leading-order version of Eq. (6.14) determines $\nu^{(-1)}$ as

$$
\nu^{(-1)}=0, \quad \zeta<-\left(x_{r}^{0}-x_{m}^{0}\right)_{0}
$$

Consequently, Eq. (8.1) may be solved to obtain the solution for $\sigma^{(0)}$ in this region as

$$
\sigma^{(0)}=c_{1} e^{\bar{q}_{+}^{(0)} \zeta}, \quad \zeta<-\left(x_{r}^{0}-x_{m}^{0}\right)_{0}
$$

where $\bar{q}_{+}^{(0)}=\frac{1}{2}\left[1+\sqrt{1+4\left(i \omega_{0}+k^{2}\right)}\right]$.

At the next order in $\epsilon$, Eq. (6.13) gives, after some simplification,

$$
\begin{aligned}
i \omega_{0} \sigma^{(1)}+i \omega_{1} \sigma^{(0)}+ & \frac{d \sigma^{(1)}}{d \zeta}-\frac{d^{2} \sigma^{(1)}}{d \zeta^{2}}+k^{2} \sigma^{(1)}=\frac{\alpha_{s} \hat{l}^{*}}{1-\alpha_{s}}\left(\frac{d^{2} \sigma^{(0)}}{d \zeta^{2}}-k^{2} \sigma^{(0)}\right) \\
& +\frac{\hat{r}^{*} \hat{b} \alpha_{s}}{1-\alpha_{s}}\left(\chi \frac{d \nu^{(0)}}{d \zeta}+i \omega_{0} \frac{d T_{0}}{d \zeta}-i \omega_{0} \chi \frac{d q_{0}}{d \zeta}\right), \quad \zeta<-\left(x_{r}^{0}-x_{m}^{0}\right)_{0}
\end{aligned}
$$

where $q_{0}$ is the leading-order coefficient in the expansion (7.1e) of the basic solution for the overpressure in the outer portion of the solid/gas region, and we note from Eqs. (5.11) at leading order that $d p_{0} / d \zeta=d T_{0} / d \zeta$. The leading-order basic solution, which is briefly derived in Appendix A, 
is given by $T_{0}=\left(1+q_{0}\right)=1+\left(T_{m}-1\right) e^{\zeta+\left(x_{r}^{0}-x_{m}^{0}\right)_{0}}$. Using the next-order version of Eq. (6.14) to determine that $\nu^{(0)}=\sigma^{(0)}$ and substituting the results obtained thus far into Eq. (8.4), we find that the solution for $\sigma^{(1)}$ is determined as

$$
\sigma^{(1)}=\hat{c}_{1} e^{\bar{q}_{+}^{(0)} \zeta}-\frac{L_{1}}{2 q_{+}^{(0)}-1} \zeta e^{\bar{q}_{+}^{(0)} \zeta}+\frac{L_{2}}{i \omega_{0}+k^{2}} e^{\zeta}, \quad \zeta<-\left(x_{r}^{0}-x_{m}^{0}\right)_{0}
$$

where $L_{1}$ and $L_{2}$ are given by

$$
\begin{aligned}
& L_{1}=\left\{\frac{\alpha_{s}}{1-\alpha_{s}}\left[\hat{l}^{*}\left(i \omega_{0}+\bar{q}_{+}^{(0)}\right)+\hat{r}^{*} \hat{b}(\chi-1) q_{+}^{(0)}\right]-i \omega_{1}\right\} c_{1} \\
& L_{2}=-\frac{\alpha_{s}}{1-\alpha_{s}} \hat{r}^{*} \hat{b}(\chi-1) i \omega_{0}\left(T_{m}-1\right) e^{\zeta+\left(x_{r}^{0}-x_{m}^{0}\right)_{0}}
\end{aligned}
$$

We now consider the boundary-layer solution in the solid/gas region adjacent to the melting surface, which is needed to connect the outer solutions given above with those in the liquid/gas region. From Eq. (6.13), the leading-order equation for the temperature perturbation is obtained as

$$
\frac{d^{2} \sigma_{b l}^{(0)}}{d \hat{\eta}^{2}}=0
$$

where $\sigma_{b l}$ is the leading-order temperature-perturbation variable in the boundary layer. The solution to Eq. (8.6) that matches with the outer solution (8.3) as $\hat{\eta} \rightarrow-\infty$ is a constant value

$$
\sigma_{b l}^{(0)}=c_{1} e^{\bar{q}_{+}^{(0)}\left(x_{r}^{0}-x_{m}^{0}\right)_{0}}
$$

reflecting the fact that, to a first approximation, the gas-permeation layer is a boundary layer with respect to pressure, and not temperature.

The next-order equation for the boundary-layer temperature perturbation is obtained from Eq. (6.13) and Eq. (8.7) as

$$
\frac{d^{2} \sigma_{b l}^{(1)}}{d \hat{\eta}^{2}}=-\frac{\hat{r}^{*} \hat{b} \alpha_{s} \chi}{1-\alpha_{s}} \frac{d \nu_{b l}^{(-1)}}{d \hat{\eta}}
$$

which indicates the first coupling of the temperature- and pressure-perturbation variables. To solve Eq. (8.8) for $\sigma_{b l}^{(1)}$ we need the expression for $\nu_{b l}^{(-1)}$, which is obtained from the leading-order version of Eq. (6.14) and the last of Eqs. (6.19) in the boundary layer as

$$
\frac{\kappa^{*}}{\alpha_{s}}\left(p_{0}+1\right) \frac{d \nu_{b l}^{(-1)}}{d \hat{\eta}}+\frac{\kappa^{*}}{\alpha_{s}} \frac{d p_{0}}{d \hat{\eta}} \nu_{b l}^{(-1)}=0
$$

subject to

$$
\left.\left(\nu_{b l}^{(-1)}+c_{m}^{(0)} \frac{d p_{0}}{d \hat{\eta}}\right)\right|_{\hat{\eta}=0}=0
$$


Here, $p_{0}$ is the leading-order overpressure in the expansions (7.4) of the basic solution in the boundary layer, and $p_{b}=p_{g}^{b}-1$ is its value at the melting surface $\hat{\eta}=0$. This is given implicitly (see Appendix A) by

$$
\frac{\alpha_{s}}{\kappa^{*}} \hat{\eta}=p_{0}-p_{b}+T_{m} \ln \left(\frac{p_{0}-T_{m}+1}{p_{b}-T_{m}+1}\right)
$$

so that

$$
\frac{d p_{0}}{d \hat{\eta}}=\frac{\alpha_{s}}{\kappa^{*}}\left(\frac{p_{0}-T_{m}+1}{p_{b}-T_{m}+1}\right) .
$$

With these results, $p_{0}$ can be employed as the independent variable in Eq. (8.9) to give

$$
\nu_{b l}^{(-1)}\left(p_{0}\right)=-\frac{\alpha_{s}}{\kappa^{*}} c_{m}^{(0)} \frac{p_{0}-T_{m}+1}{p_{b}-T_{m}+1}
$$

Having thus obtained an expression for $\nu_{b l}^{(-1)}$ in terms of $p_{0}$, we can solve Eq. (8.8) to obtain the solution for $\sigma_{b l}^{(1)}$ as

$$
\sigma_{b l}^{(1)}=\hat{A}_{1} \hat{\eta}+\hat{A}_{2}+\frac{\hat{r}^{*} \hat{b} \chi \alpha_{s}}{1-\alpha_{s}} c_{m}^{(0)} p_{0}
$$

where $\hat{A}_{1}$ and $\hat{A}_{2}$ are constants of integration that are obtained from a (2,2) matching (cf. [21]) with the outer solution in the solid/gas region. Using Eqs. (8.3), (8.5), (8.7) and (8.14), the result of this procedure is that $\sigma_{b l}^{(1)}$ is given by

$$
\begin{aligned}
\sigma_{b l}^{(1)}=c_{1} \bar{q}_{+}^{(0)} \hat{\eta} e^{-\bar{q}_{+}^{(0)}\left(x_{r}^{0}-x_{m}^{0}\right)_{0}}+\left(\hat{c}_{1}+L_{1} \frac{\left(x_{r}^{0}-x_{m}^{0}\right)_{0}}{2 \bar{q}_{+}^{(0)}-1}\right) e^{-\bar{q}_{+}^{(0)}\left(x_{r}^{0}-x_{m}^{0}\right)_{0}} \\
\\
\quad-\frac{\alpha_{s}}{1-\alpha_{s}}\left(T_{m}-1\right) \hat{r}^{*} \hat{b}(\chi-1) \frac{i \omega_{0}}{i \omega_{0}+k^{2}}+\frac{\alpha_{s}}{1-\alpha_{s}} \hat{r}^{*} \hat{b} c_{m}^{(0)} \chi\left(p_{0}-T_{m}+1\right)
\end{aligned}
$$

with $L_{1}$ given by Eq. (8.5b).

Continuing our perturbation analysis with a consideration of the liquid/gas region, we find that the leading-order approximation of Eq. (6.15) yields

$$
\frac{d^{2} \sigma^{(0)}}{d \zeta^{2}}-\frac{b}{l} \frac{d \sigma^{(0)}}{d \zeta}-\left(\frac{r b}{l} i \omega_{0}+k^{2}\right) \sigma^{(0)}+\frac{b}{l}(1-r) \frac{d T_{0}}{d \zeta} i \omega_{0} c_{m}^{(0)}=0, \quad-\left(x_{r}^{0}-x_{m}^{0}\right)_{0}<\zeta<0
$$

This has the solution

$$
\sigma^{(0)}=c_{2} e^{q_{-}^{(0)} \zeta}+c_{3} e^{q_{+}^{(0)} \zeta}+\frac{(b / l)(1-r)\left(b T_{m}-1\right) i \omega_{0} c_{m}^{(0)}}{i \omega_{0} r b+l k^{2}} e^{(b / l)\left(\zeta+\left(x_{r}^{0}-x_{m}^{0}\right)_{0}\right.}, \quad-\left(x_{r}^{0}-x_{m}^{0}\right)_{0}<\zeta<0,
$$

where $2 q_{ \pm}^{(0)}=\left\{b / l \pm\left[(b / l)^{2}+4\left(i \omega_{0} r b / l+k^{2}\right)\right]^{1 / 2}\right\}$. Finally, in the burned region $\zeta>0$, the leading-order version of Eq. (6.16) gives

$$
\hat{b}\left(1-\alpha_{s}\right) \frac{d \sigma^{(0)}}{d \zeta}=0, \quad \zeta>0
$$


from which we obtain

$$
\sigma^{(0)}=c_{4}, \quad \zeta>0
$$

The determination of the constants of integration in the solutions obtained thus far, as well as the leading-order growth rate $i \omega_{0}$, follows from the boundary conditions given by Eqs. (6.18) (6.22). We observe that since the boundary layer in the solid/gas region is adjacent to the melting surface, the jump conditions across the melting surface are with respect to the solutions obtained in that layer, so that in Eq. (6.20) for example,

$$
\left.\left.\left(\frac{d \sigma}{d \zeta}+c_{m} \frac{d^{2} T^{0}}{d \zeta^{2}}\right)\right|_{\zeta=-\left(x_{r}^{0}-x_{m}^{0}\right)^{-}} \longrightarrow\left(\epsilon^{-1} \frac{d \sigma_{b l}}{d \hat{\eta}}+\epsilon^{-2} c_{m} \frac{d^{2} \hat{T}^{0}}{d \hat{\eta}^{2}}\right)\right|_{\hat{\eta}=0}
$$

with $\sigma_{b l}$ and $\hat{T}^{0}$ given by their respective boundary-layer expansions. Also, for algebraic simplicity in analyzing the resulting dispersion relations, we now restrict further consideration to a representative case in which differences in the physical properties of the solid and liquid phases are neglected. In particular, we consider, as in $[8,9]$, the case in which $r=b=l=1$ so that, for example, $\bar{q}_{+}=q_{+}$. We note that $O(\epsilon)$ deviations in these equalities, which will not affect the leading-order dispersion relation, can easily be incorporated into the present analysis to give tractable corrections at the next order (Section 9).

Thus, to obtain the leading-order dispersion relation with respect to $\epsilon$, we apply the corresponding leading-order jump conditions (6.18) - (6.22) across the melting surface and reaction sheet. Using the fact [see Eqs. (9.1) below] that $d \hat{T}_{1} /\left.d \hat{\eta}\right|_{\hat{\eta}=0}=\left(T_{m}-1\right)$ and $d^{2} \hat{T}_{2} /\left.d \hat{\eta}^{2}\right|_{\hat{\eta}=0}=$ $T_{m}-1-\alpha_{s}\left(1-\alpha_{s}\right)^{-1} \hat{r}^{*} \hat{b} \chi\left(\alpha_{s} / \kappa^{*}\right)\left(p_{b}-T_{m}+1\right) /\left(p_{b}+1\right)$, which are needed in the evaluation of Eqs. (6.19) and (6.20) on the boundary-layer side of the solid/liquid interface, we obtain

$$
\begin{aligned}
& c_{1}=-c_{m}^{(0)}\left(T_{m}-1\right) e^{q_{+}^{0}\left(x_{r}^{0}-x_{m}^{0}\right)_{0}}, \quad c_{2}=0, \\
& c_{3}=-c_{m}^{(0)}\left(T_{m}-1\right) e^{q_{+}^{0}\left(x_{r}^{0}-x_{m}^{0}\right)_{0}}, \quad c_{4}=-2 i \omega_{0} \frac{T_{b}^{0}-1}{\beta_{0}}
\end{aligned}
$$

where, $\hat{c}_{1}$ is not needed since only $d \sigma_{b l}^{(1)} /\left.d \hat{\eta}\right|_{\hat{\eta}=0}$ is required in the present leading-order calculation. Here, from Eq. (5.8),

$$
\left(x_{r}^{0}-x_{m}^{0}\right)_{0}=\ln \left(\frac{T_{b}^{0}-1}{T_{m}-1}\right)
$$

and $c_{m}^{(0)}$ is given by

$$
c_{m}^{(0)}=\frac{\left(\beta_{0}+2 i \omega_{0}\right)\left(T_{b}^{0}-1\right)}{\beta_{0}\left(T_{m}-1\right)} e^{q_{+}^{0}\left(x_{r}^{0}-x_{m}^{0}\right)_{0}},
$$

where the leading-order expression for the burned temperature is obtained from Eq. (3.15) as

$$
T_{b}^{0}=\frac{Q_{0}+1}{\hat{b}}
$$


Finally, applying Eq. (6.22) at leading order gives

$$
q_{+}^{0} c_{3}+\left(T_{b}^{0}-1\right)=\left(\beta_{0} \frac{Q_{0}+(1-\hat{b}) T_{b}^{0}}{2\left(T_{b}^{0}-1\right)}+1-\hat{b}\right) c_{4}
$$

which, from the above expressions for $c_{3}, c_{4}$ and $q_{+}^{(0)}$ (with $b=l=1$ ), becomes

$$
\left(2 i \omega_{0}+\beta_{0}\right)\left(1-q_{+}^{0}\right)+i \omega_{0}\left(\beta_{0}-2 \hat{b}\right)=0
$$

Equation (8.25) is identical to that obtained in the absence of confinement $[8,9]$, indicating that, in the parameter regime (7.1a), the effects of confinement are not felt at leading order. The neutral stability boundary, obtained by setting the real part of the complex growth rate $i \omega_{0}$ to zero, determines the (pulsating) neutral stability boundary as the positive root of the quadratic

$$
\left(\hat{b}+2 k^{2}\right) \beta_{0}^{2}+2\left[k^{2}-(1+2 \hat{b})\left(\hat{b}+2 k^{2}\right)\right] \beta_{0}-4\left(\hat{b}+2 k^{2}\right)\left[\hat{b}(1-\hat{b})+k^{2}\right]=0
$$

where the corresponding leading-order frequency $\omega_{0}(k)$ of the neutral disturbance is

$$
\omega_{0}^{2}=\frac{1}{2} \beta_{0}\left(\hat{b}+2 k^{2}\right)
$$

This is a manifestation of a well known oscillatory condensed-phase instability [cf. 17,19,20] and is discussed in more detail below. The neutral stability boundary appears as the solid curve in Figures 2a-f. As in the case of the unconfined problem [8,9], effects arising from porosity and two-phase flow, as well as those associated with confinement, appear at the next order in the analysis. It is thus necessary to continue with the above analysis in order to calculate the correction coefficient $i \omega_{1}$. Setting the real part of $i \omega_{1}$ to zero will then determine the corresponding modifications to the neutral stability boundary associated with pressure-driven gas permeation into the unburned porous solid.

\section{Correction to the Leading-Order Dispersion Relation}

Before continuing with the analysis, we observe that consideration of higher-order problems for the perturbation coefficients in Eqs. (7.1f) and (7.3) necessarily involve higher-order solutions for the basic solution as well. In the region $\zeta>0$, these coefficients are obtained by simply expanding the basic solution (5.9), but in the region $\zeta<0$, and particularly in the boundary-layer portion of this region, solutions must be obtained formally by solving the sequence of problems obtained from substituting the expansions and scalings directly into Eqs. (5.11) and the associated boundary conditions. This analysis is described in Appendix A, where, for example, the expressions for the boundary-layer overpressure coefficients $p_{0}$ and $p_{1}$ are given in Eqs. (A.5) and (A.8). Fortunately, 
some of the higher-order temperature coefficients are only required as derivatives evaluated at the melting surface $\hat{\eta}=0$. From Eqs. $(5.11 \mathrm{a}, \mathrm{b})$, these quantities can be determined, without calculating the functions themselves, as

$$
\begin{aligned}
& \hat{T}_{0}=T_{m}, \quad \frac{d \hat{T}_{0}}{d \hat{\eta}}=\frac{d^{2} \hat{T}_{0}}{d \hat{\eta}^{2}}=0,\left.\quad \frac{d \hat{T}_{1}}{d \hat{\eta}}\right|_{\hat{\eta}=0}=\left(T_{m}-1\right),\left.\quad \frac{d^{2} \hat{T}_{1}}{d \hat{\eta}^{2}}\right|_{\hat{\eta}=0}=0, \\
& \left.\frac{d \hat{T}_{2}}{d \hat{\eta}}\right|_{\hat{\eta}=0}=\frac{\alpha_{s}}{1-\alpha_{s}}\left[\hat{r}^{*} \hat{b}\left(T_{m}-1-\chi p_{b}\right)-\left(T_{m}-1\right) \hat{l}^{*}\right], \\
& \left.\frac{d^{2} \hat{T}_{2}}{d \hat{\eta}^{2}}\right|_{\hat{\eta}=0}=\left(T_{m}-1\right)-\frac{\alpha_{s}^{2}}{\kappa^{*}\left(1-\alpha_{s}\right)} \hat{r}^{*} \hat{b} \chi \frac{p_{b}-T_{m}+1}{p_{b}+1}, \\
& \left.\frac{d^{2} \hat{T}_{3}}{d \hat{\eta}^{2}}\right|_{\hat{\eta}=0}=\frac{\alpha_{s}}{1-\alpha_{s}}\left[2\left(T_{m}-1\right)\left(\hat{r}^{*} \hat{b}-\hat{l}^{*}\right)+\hat{r}^{*} \hat{b} \chi\left(\frac{\alpha_{s}^{2}}{\kappa^{*}\left(1-\alpha_{s}\right.} \hat{l}^{*} \frac{p_{b}-T_{m}+1}{p_{b}+1}-p_{b}\right)\right],
\end{aligned}
$$

where the $\hat{T}_{i}$ are the coefficients in the boundary-layer expansion (7.4) of the basic temperature $T^{0}$.

To obtain the $O(\epsilon)$ dispersion relation $i \omega_{1}(k)$ it is necessary to consider the equations and boundary conditions at a higher order in all regions and across all interfaces. First, from the jump conditions (6.19) and (6.20) at the melting surface, it is clear that the solution for $\sigma^{(2)}(\zeta)$ is required in the form of an expression for $d \sigma_{b l}^{(2)} /\left.d \hat{\eta}\right|_{\hat{\eta}=0}$. This entails solving Eq. (6.13) in both the outer and boundary-layer portions of the solid/gas region. In the outer region, we thus collect terms of $\mathrm{O}\left(\epsilon^{2}\right)$ to obtain an equation for $\sigma^{(2)}(\zeta)$, which involves the coefficient $\nu^{(1)}$. The latter, specifically $d \nu^{(1)} / d \zeta$, is determined from the $O(\epsilon)$ terms in Eq. (6.14), which is shown in Appendix $\mathrm{B}$ to give rise to the result

$$
\frac{d \nu^{(1)}}{d \zeta}=N_{1} e^{q_{+}^{(0)} \zeta}+\zeta N_{2} e^{q_{+}^{(0)} \zeta}+N_{3} e^{\zeta}+N_{4} e^{2 \zeta}+N_{5} e^{\left[q_{+}^{(0)}+1\right] \zeta}, \quad \zeta<-\left(x_{r}^{0}-x_{m}^{0}\right)_{0},
$$

where the constant coefficients $N_{i}$ are given in Appendix B. The equation for the temperature perturbation coefficient $\sigma^{(2)}$ is then obtained as

$$
\begin{gathered}
\frac{d \sigma^{(2)}}{d \zeta}+\left(i \omega_{0}+k^{2}\right) \sigma^{(2)}-\frac{d^{2} \sigma^{(2)}}{d \zeta^{2}}=-i \omega_{1} \sigma^{(1)}-\frac{\alpha_{s}}{1-\alpha_{s}}\left[\hat{r}^{*} \hat{b} \frac{d \sigma^{(1)}}{d \zeta}+\hat{l}^{*}\left(k^{2} \sigma^{(1)}-\frac{d^{2} \sigma^{(1)}}{d \zeta^{2}}\right)\right] \\
-i \omega_{2} \sigma^{(0)}+\frac{\hat{r}^{*} \hat{b} \chi \alpha_{s}}{1-\alpha_{s}} \frac{d \nu^{(1)}}{d \zeta}+\frac{\hat{r}^{*} \hat{b} \alpha_{s}}{1-\alpha_{s}}\left[i \omega_{0} \frac{d T_{1}}{d \zeta}+i \omega_{1} \frac{d T_{0}}{d \zeta}-\chi\left(i \omega_{0} \frac{d q_{1}}{d \zeta}+i \omega_{1} \frac{d q_{0}}{d \zeta}\right)\right]
\end{gathered}
$$

where the $q_{i}$ are coefficients in the expansion (7.1e) of the basic solution for overpressure in the region $\zeta<-\left(x_{r}^{0}-x_{m}^{0}\right)$. Using the result (9.2) the solution for $\sigma^{(2)}$ is given by

$$
\sigma^{(2)}=\tilde{c}_{1} e^{q_{+}^{(0)} \zeta}+S_{1} \zeta e^{q_{+}^{(0)} \zeta}+S_{2} \zeta^{2} e^{q_{+}^{(0)} \zeta}+S_{3} e^{\zeta}+S_{4} \zeta e^{\zeta}+S_{5} e^{2 \zeta}+S_{6} e^{\left[q_{+}^{(0)}+1\right] \zeta}, \quad \zeta<-\left(x_{r}^{0}-x_{m}^{0}\right)_{0},
$$

where the constant coefficients $S_{i}$ also are given in Appendix B. 
To solve the corresponding equation for $\sigma^{(2)}$ in the boundary layer, we collect terms in Eq. (6.13) that are $\mathrm{O}(1)$ there, which involve the coefficient $\nu_{b l}^{(0)}$. From the boundary-layer version of Eq. (6.14) and the last of Eqs. (6.19), the problem for $\nu_{b l}^{(0)}$ is given by

$$
\begin{gathered}
\frac{\kappa^{*}}{\alpha_{s}}\left(p_{0}+1\right) \frac{d \nu_{b l}^{(0)}}{d \hat{\eta}}+\frac{\kappa^{*}}{\alpha_{s}} p_{1} \frac{d \nu_{b l}^{(-1)}}{d \hat{\eta}}+\frac{\kappa^{*}}{\alpha_{s}} \frac{d p_{0}}{d \hat{\eta}} \nu_{b l}^{(0)}+\frac{\kappa^{* 2}}{\alpha_{s}^{2}} i \omega_{0} \frac{d p_{1}}{d \hat{\eta}} \nu_{b l}^{(-1)}\left(p_{0}+1\right) \frac{d p_{0}}{d \hat{\eta}}-\nu_{b l}^{(0)}+\sigma_{b l}^{(0)}=0 \\
\left.\left(\nu_{b l}^{(0)}+c_{m}^{(0)} \frac{d p_{1}}{d \hat{\eta}}+c_{m}^{(1)} \frac{d p_{0}}{d \hat{\eta}}\right)\right|_{\hat{\eta}=0}=0
\end{gathered}
$$

where $p_{1}$ is given as a function of $p_{0}$ by Eq. (A.8). The resulting solution for $\nu_{b l}^{(0)}\left(p_{0}\right)$ is then given by

$$
\begin{aligned}
\nu_{b l}^{(0)}= & -\frac{\alpha_{s}}{\kappa^{*}} c_{m}^{(0)} \frac{T_{m}}{\left(p_{0}+1\right)^{2}} p_{1}\left(p_{0}\right) \\
& -\left(\frac{p_{0}-T_{m}+1}{p_{0}+1}\right)\left\{\left(i \omega_{0}-c_{m}^{(0)} \frac{T_{m}-1}{p_{0}-T_{m}+1}\right)\left[p_{0}-p_{b}+T_{m} \ln \left(\frac{p_{0}-T_{m}+1}{p_{b}-T_{m}+1}\right)\right]+c_{m}^{(1)} \frac{\alpha_{s}}{\kappa^{*}}\right\} .
\end{aligned}
$$

With this result, the equation for $\sigma_{b l}^{(2)}$, which is given by

$$
\begin{aligned}
& \frac{d^{2} \sigma_{b l}^{(2)}}{d \hat{\eta}^{2}}=\left(i \omega_{0}+k^{2}\right) \sigma_{b l}^{(0)}+\frac{d \sigma_{b l}^{(1)}}{d \hat{\eta}} \\
& \quad+\frac{\alpha_{s}}{1-\alpha_{s}}\left[\hat{r}^{*} \hat{b}\left(\frac{d \sigma_{b l}^{(0)}}{d \hat{\eta}}-\chi \frac{d \nu_{b l}^{(0)}}{d \hat{\eta}}\right)-\hat{l}^{*} \frac{d^{2} \sigma_{b l}^{(1)}}{d \hat{\eta}^{2}}-i \omega_{0} \hat{r}^{*} \hat{b}\left(\frac{d T_{0}}{d \hat{\eta}}-\chi \frac{d p_{0}}{d \hat{\eta}}\right)\right],
\end{aligned}
$$

can be solved subject to a matching with the outer solution (Appendix B). The resulting solution can then be differentiated and evaluated to provide the required result

$$
\begin{aligned}
\left.\frac{d \sigma_{b l}^{(2)}}{d \hat{\eta}}\right|_{\hat{\eta}=0}= & -\frac{\alpha_{s}^{2}}{\kappa^{*}\left(1-\alpha_{s}\right)} \hat{r}^{*} \hat{b} \chi \frac{p_{b}-T_{m}+1}{p_{b}+1}\left(\hat{l}^{*} c_{m}^{(0)}+\frac{\alpha_{s}}{1-\alpha_{s}} c_{m}^{(1)}\right) \\
& +\frac{\alpha_{s}}{1-\alpha_{s}} \hat{r}^{*} \hat{b}\left[\chi\left(c_{m}^{(0)}+i \omega_{0}\right) p_{b}-(\chi-1)\left(T_{m}-1\right) \frac{i \omega_{0}}{i \omega_{0}+k^{2}}\right] \\
& -\frac{\alpha_{s}}{1-\alpha_{s}} c_{m}^{(0)}\left(T_{m}-1\right) \hat{r}^{*} \hat{b} \chi\left(\frac{i \omega_{0}+c_{m}^{(0)}}{T_{m} c_{m}^{(0)}}+1\right)+q_{+}^{0} \hat{c}_{1} e^{q_{+}^{0}\left(x_{r}^{0}-x_{m}^{0}\right)_{0}} \\
& +\frac{c_{m}^{(0)}\left(T_{m}-1\right)\left[1-q_{+}^{0}\left(x_{r}^{0}-x_{m}^{0}\right)_{0}\right]}{2 q_{+}^{0}-1}\left\{\frac{\alpha_{s}}{1-\alpha_{s}}\left[\hat{l}^{*}\left(i \omega_{0}+q_{+}^{0}\right)+\hat{r}^{*} \hat{b}(\chi-1) q_{+}^{0}\right]-i \omega_{1}\right\},
\end{aligned}
$$

where $c_{m}^{(1)}$ and the integration constant $\hat{c}_{1}$ are to be determined.

We now proceed to consider the next-order temperature-perturbation equations in the liquid/gas and burned-gas regions. From Eqs. (6.15) and (6.16) with $r=b=l=1$, we obtain at $\mathrm{O}(\epsilon)$ the equations for $\sigma^{(1)}$ as

$$
\begin{aligned}
\left(1-\alpha_{s}\right)\left[\left(i \omega_{0}+k^{2}\right) \sigma^{(1)}\right. & \left.+\frac{d \sigma^{(1)}}{d \zeta}-\frac{d^{2} \sigma^{(1)}}{d \zeta^{2}}\right]=-\left(1-\alpha_{s}\right) i \omega_{1} \sigma^{(0)}-\alpha_{s} \hat{r}^{*} \hat{b} \frac{d \sigma^{(0)}}{d \zeta} \\
& +\alpha_{s} \hat{l}^{*}\left(\frac{d^{2} \dot{\sigma}^{(0)}}{d \zeta^{2}}-k^{2} \sigma^{(0)}\right)+\alpha_{s} \hat{r}^{*} \hat{b} \frac{d T_{0}}{d \zeta} i \omega_{0}, \quad-\left(x_{r}^{0}-x_{m}^{0}\right)_{0}<\zeta<0
\end{aligned}
$$




$$
\hat{b}\left(1-\alpha_{s}\right) \frac{d \sigma^{(1)}}{d \zeta}+\hat{l}^{*}\left(k^{2} \sigma^{(1)}-\frac{d^{2} \sigma^{(1)}}{d \zeta^{2}}\right)=-\hat{r}^{*} \hat{b} \alpha_{s} \frac{d \sigma^{(0)}}{d \zeta}, \quad \zeta>0
$$

Consequently, the temperature perturbation $\sigma^{(1)}$ is expressed as

$$
\sigma^{(1)}=\hat{c}_{2} e^{q_{-}^{(0)} \zeta}+\hat{c}_{3} e^{q_{+}^{(0)} \zeta}-\zeta \frac{M_{1}}{2 q_{+}^{0}-1} e^{q_{+}^{(0)} \zeta}+\frac{M_{2}}{i \omega_{0}+k^{2}} e^{\zeta}, \quad-\left(x_{r}^{0}-x_{m}^{0}\right)_{0}<\zeta<0,
$$

and

$$
\sigma^{(1)}=\frac{\hat{l}^{*} k^{2}}{\hat{b}\left(1-\alpha_{s}\right)} \frac{2 i \omega_{0}}{\beta_{0}}\left(T_{b}^{0}-1\right) \zeta+\hat{c}_{4}, \quad \zeta>0
$$

where $M_{1}$ and $M_{2}$ are given by

$$
\begin{aligned}
& M_{1}=c_{m}^{(0)}\left(T_{m}-1\right) e^{q_{+}^{0}\left(x_{r}^{0}-x_{m}^{0}\right)_{0}}\left(i \omega_{1}+\frac{\alpha_{s}}{1-\alpha_{s}}\left[\hat{r}^{*} \hat{b} q_{+}^{(0)}-\hat{l}^{*}\left(i \omega_{0}+q_{+}^{(0)}\right)\right]\right), \\
& M_{2}=\frac{\alpha_{s}}{1-\alpha_{s}} \hat{r}^{*} \hat{b} i \omega_{0}\left(T_{m}-1\right) e^{\left(x_{r}^{0}-x_{m}^{0}\right)_{0}},
\end{aligned}
$$

and the integration constants $\hat{c}_{i}$ are to be determined.

Given these results, $c_{m}^{1}$ and the $\hat{c}_{i}$ are determined from the jump conditions $(6.18)-(6.21)$ at the appropriate order with respect to $\epsilon$. We thus obtain

$$
\begin{aligned}
& \hat{c}_{1}=\left\{-\left(T_{m}-1\right)\left[\frac{\left(x_{r}^{0}-x_{m}^{0}\right)_{0} c_{m}^{(0)} i \omega_{1}}{2 q_{+}^{0}-1}+c_{m}^{(1)}\right]-\left(T_{m}-1\right) c_{m}^{(0)} q_{+}^{0}\left(x_{r}^{0}-x_{m}^{0}\right)_{1}\right\} e^{q_{+}^{0}\left(x_{r}^{0}-x_{m}^{0}\right)_{0}} \\
& +\frac{\alpha_{s}}{1-\alpha_{s}}\left\{\frac{\left(x_{r}^{0}-x_{m}^{0}\right)_{0} c_{m}^{(0)}\left(T_{m}-1\right)}{2 q_{+}^{0}-1}\left[\hat{\imath}^{*}\left(i \omega_{0}+q_{+}^{0}\right)+r^{*} \hat{b}(\chi-1) q_{+}^{0}\right]\right. \\
& +\left(T_{m}-1\right) \hat{r}^{*} \hat{b}(\chi-1) \frac{i \omega_{0}}{i \omega_{0}+k^{2}}-r^{*} \hat{b} c_{m}^{(0)} \chi\left(p_{b}-T_{m}+1\right) \\
& \left.-c_{m}^{(0)}\left[\hat{r}^{*} \hat{b}\left(T_{m}-1-\chi p_{b}\right)-\left(T_{m}-1\right) \hat{l}^{*}\right]\right\} e^{q_{+}^{\mathrm{o}}\left(x_{r}^{\mathrm{o}}-x_{m}^{\mathrm{o}}\right)_{0}}, \\
& \hat{c}_{2}=\left[-c_{m}^{(0)} \gamma_{s}^{*} \frac{q_{-}^{0}+i \omega_{0}}{q_{+}^{0}-q_{-}^{0}}+c_{m}^{(0)}\left(T_{m}-1\right) \frac{q_{+}^{0} q_{+}^{0}}{q_{+}^{0}-q_{-}^{0}}\left(x_{r}^{0}-x_{m}^{0}\right)_{1}\right] e^{q_{-}^{0}\left(x_{r}^{0}-x_{m}^{0}\right)_{o}} \\
& +\frac{\alpha_{s}}{1-\alpha_{s}}\left\{\left(T_{m}-1\right) \hat{r}^{*} \hat{b} \chi q_{-}^{0} \frac{i \omega_{0}}{i \omega_{0}+k^{2}}+q_{+}^{0} r^{*} \hat{b} c_{m}^{(0)} \chi\left(p_{b}-T_{m}+1\right)-c_{m}^{(0)}\left(T_{m}-1\right) \frac{q_{+}^{0}}{2 q_{+}^{0}-1} \hat{r}^{*} \hat{b} \chi\right. \\
& \left.+\hat{r}^{*} \hat{b} \chi\left[\left(c_{m}^{(0)}+i \omega_{0}\right)\left(1-1 / T_{m}-p_{b}\right)+c_{m}^{(0)}\left(T_{m}-1\right)\right]\right\} \frac{e^{q_{-}^{0}\left(x_{r}^{0}-x_{m}^{0}\right)_{o}}}{q_{+}^{0}-q_{-}^{0}},
\end{aligned}
$$




$$
\begin{aligned}
& \hat{c}_{3}=\left[c_{m}^{(0)} \gamma_{s}^{*} \frac{q_{+}^{0}+i \omega_{0}}{q_{+}^{0}-q_{-}^{0}}-c_{m}^{(0)}\left(T_{m}-1\right) i \omega_{1} \frac{\left(x_{r}^{0}-x_{m}^{0}\right)_{0}}{2 q_{+}^{0}-1}\right. \\
& \left.-c_{m}^{(0)}\left(T_{m}-1\right)\left(x_{r}^{0}-x_{m}^{0}\right)_{1} q_{+}^{0} \frac{2 q_{+}^{0}-q_{-}^{0}}{q_{+}^{0}-q_{-}^{0}}\right] e^{q_{+}^{0}\left(x_{r}^{0}-x_{m}^{0}\right)_{0}}-c_{m}^{(1)}\left(T_{m}-1\right) e^{q_{+}^{0}\left(x_{r}^{0}-x_{m}^{0}\right)_{0}} \\
& -\frac{\alpha_{s}}{1-\alpha_{s}}\left\{\frac{\left(x_{r}^{0}-x_{m}^{0}\right)_{0} c_{m}^{(0)}\left(T_{m}-1\right)}{2 q_{+}^{0}-1}\left[r^{*} \hat{b} q_{+}^{0}-\hat{l}^{*}\left(i \omega_{0}+q_{+}^{0}\right)\right]\left(q_{+}^{0}-q_{-}^{0}\right)\right. \\
& +\left(T_{m}-1\right) \hat{r}^{*} \hat{b}\left[q_{-}^{0}(\chi-1)+q_{+}^{0}\right] \frac{i \omega_{0}}{i \omega_{0}+k^{2}}-c_{m}^{(0)} q_{-}^{0}\left[\left(T_{m}-1\right)\left(\hat{r}^{*} \hat{b}-\hat{l}^{*}\right)-\hat{r}^{*} \hat{b} \chi p_{b}\right] \\
& -c_{m}^{(0)}\left(T_{m}-1\right) \frac{q_{+}^{0}}{2 q_{+}^{0}-1} \hat{r}^{*} \hat{b} \chi+c_{m}^{(0)}\left(T_{m}-1\right) q_{+}^{0}\left[\hat{r}^{*} \hat{b}(1-\chi)-\hat{l}^{*}\right] \\
& \left.+\hat{r}^{*} \hat{b} \chi\left[\left(c_{m}^{(0)}+i \omega_{0}\right)\left(1-T_{m}^{-1}-p_{b}\right)+c_{m}^{(0)}\left(T_{m}-1\right)\right]\right\} \frac{e^{q_{+}^{0}\left(x_{r}^{0}-x_{m}^{0}\right)_{0}}}{q_{+}^{0}-q_{-}^{0}} \\
& \hat{c}_{4}=\frac{2\left(T_{b}^{0}-1\right)}{\beta_{0}}\left[\frac{\beta_{1}}{\beta_{0}} i \omega_{0}-i \omega_{1}-\frac{\alpha_{s}}{1-\alpha_{s}} \hat{r}^{*} \frac{\left(T_{b}^{0}-1-\chi p_{b}\right)}{\left(T_{b}^{0}-1\right)} i \omega_{0}\right]
\end{aligned}
$$

and

$$
\begin{aligned}
& c_{m}^{(1)}=\left[-\frac{2\left(T_{b}^{0}-1\right)}{\beta_{0}^{2}\left(T_{m}-1\right)} i \omega_{0} \beta_{1}+\frac{\left(T_{m}-1\right)\left(x_{r}^{0}-x_{m}^{0}\right)_{1}-\gamma_{s}^{*}}{\left(T_{m}-1\right)} e^{\left(x_{r}^{0}-x_{m}^{\mathrm{o}}\right)_{0}}\right] e^{-q_{+}^{\mathrm{o}}\left(x_{r}^{\mathrm{o}}-x_{m}^{\mathrm{o}}\right)_{0}} \\
& +i \omega_{1}\left[\frac{2\left(T_{b}^{0}-1\right)}{\beta_{0}\left(T_{m}-1\right)} e^{-q_{+}^{0}\left(x_{r}^{0}-x_{m}^{0}\right)_{0}}-c_{m}^{(0)} \frac{\left(x_{r}^{0}-x_{m}^{0}\right)_{0}}{2 q_{+}^{0}-1}\right] \\
& +c_{m}^{(0)} \frac{\gamma_{s}^{*}}{\left(T_{m}-1\right)\left(q_{+}^{0}-q_{-}^{0}\right)}\left[q_{+}^{0}+i \omega_{0}-\left(q_{-}^{0}+i \omega_{0}\right) e^{\left(q_{-}^{0}-q_{+}^{0}\right)\left(x_{r}^{0}-x_{m}^{0}\right)_{0}}\right] \\
& +c_{m}^{(0)} \frac{q_{+}^{0}}{q_{+}^{0}-q_{-}^{0}}\left(x_{r}^{0}-x_{m}^{0}\right)_{1}\left[q_{+}^{0} e^{\left(q_{-}^{0}-q_{+}^{0}\right)\left(x_{r}^{0}-x_{m}^{0}\right)_{0}}-\left(2 q_{+}^{0}-q_{-}^{0}\right)\right] \\
& -\frac{\alpha_{s}}{1-\alpha_{s}} \cdot \frac{1}{q_{+}^{0}-q_{-}^{0}}\left\{\frac{i \omega_{0} \hat{r}^{*} \hat{b}}{i \omega_{0}+k^{2}}\left[\left(q_{-}^{0}(\chi-1)+q_{+}^{0}\right)-\chi q_{-}^{0} e^{\left(q_{-}^{0}-q_{+}^{0}\right)\left(x_{r}^{0}-x_{m}^{0}\right)_{0}}\right]\right. \\
& +c_{m}^{(0)} q_{+}^{0}\left[\hat{r}^{*} \hat{b}(1-\chi)-\hat{l}^{*}-\hat{r}^{*} \hat{b} \chi \frac{p_{b}-T_{m}+1}{T_{m}-1} e^{\left(q_{-}^{0}-q_{+}^{0}\right)\left(x_{r}^{0}-x_{m}^{0}\right)_{0}}\right] \\
& +\hat{r}^{*} \hat{b} \chi\left[\left(c_{m}^{(0)}+i \omega_{0}\right) \frac{T_{m}\left(1-p_{b}\right)-1}{T_{m}\left(T_{m}-1\right)}-c_{m}^{(0)} \frac{q_{+}^{0}}{2 q_{+}^{0}-1}\right]\left[1-e^{\left(q_{-}^{0}-q_{+}^{0}\right)\left(x_{r}^{0}-x_{m}^{0}\right)_{0}}\right] \\
& +c_{m}^{(0)} \frac{\left(q_{+}^{0}-q_{-}^{0}\right)\left(x_{r}^{0}-x_{m}^{0}\right)_{0}}{2 q_{+}^{0}-1}\left[\hat{r}^{*} \hat{b} q_{+}^{0}-\hat{l}^{*}\left(i \omega_{0}+q_{+}^{0}\right)\right] \\
& -c_{m}^{(0)} \frac{q_{-}^{0}}{T_{m}-1}\left[\left(T_{m}-1\right)\left(\hat{r}^{*} \hat{b}-\hat{l}^{*}\right)-\hat{r}^{*} \hat{b} \chi p_{b}\right] \\
& -\left[\frac{i \omega_{0} \hat{r}^{*} \hat{b}}{i \omega_{0}+k^{2}}+\left(\hat{r}^{*} \hat{b}-\hat{l}^{*}\right)\left[1+\left(x_{r}^{0}-x_{m}^{0}\right)_{0}\right]-\frac{\hat{r}^{*} \hat{b} \chi p_{b}}{T_{m}-1}\right]\left(q_{+}^{0}-q_{-}^{0}\right) e^{\left(1-q_{+}^{0}\right)\left(x_{r}^{0}-x_{m}^{0}\right)_{0}} \\
& \left.-\frac{2 i \omega_{0} \hat{r}^{*}\left(T_{b}^{0}-1-\chi p_{b}\right)}{\beta_{0}\left(T_{m}-1\right)}\left(q_{+}^{0}-q_{-}^{0}\right) e^{-q_{+}^{0}\left(x_{r}^{0}-x_{m}^{0}\right)_{0}}\right\}
\end{aligned}
$$


where $\left(x_{r}^{0}-x_{m}^{0}\right)_{1}$ is determined by the expansion of Eq. (5.8) as

$$
\begin{aligned}
\left(x_{r}^{0}-x_{m}^{0}\right)_{1}= & \gamma_{s}^{*} \frac{\left(T_{b}^{0}-T_{m}\right)}{\left(T_{b}^{0}-1\right)\left(T_{m}-1\right)} \\
& +\frac{\alpha_{s}}{1-\alpha_{s}}\left\{\left(\hat{l}^{*}-\hat{r}^{*} \hat{b}\right) \ln \left(\frac{T_{b}^{0}-1}{T_{m}-1}\right)+\hat{r}^{*}\left[\chi p_{b} \frac{\hat{b} T_{b}^{0}-1+(1-\hat{b}) T_{m}}{\left(T_{m}-1\right)\left(T_{b}^{0}-1\right)}-1\right]\right\} .
\end{aligned}
$$

Finally, application of Eq. (6.22) at $O(\epsilon)$ and use of the above expressions for $\hat{c}_{1}, \hat{c}_{2}, \hat{c}_{3}$, $\hat{c}_{4}$ and $c_{m}^{(1)}$ gives an equation for the $\mathrm{O}(\epsilon)$ growth-rate coefficient $i \omega_{1}$. Thus, an equation for the $O(\epsilon)$ dispersion relation $i \omega_{i}(k)$, which will determine the first-order correction $\epsilon \beta_{1}$ to the neutral stability boundary when the real part of $i \omega_{1}$ is set equal to zero, is given by

$$
\begin{aligned}
& i \omega_{1}\left[\beta_{0}+2\left(1-\hat{b}-q_{+}^{0}\right)-\frac{2 i \omega_{0}+\beta_{0}}{2 q_{+}^{0}-1}\right]+\frac{2 i \omega_{0}}{\beta_{0}}\left(q_{+}^{0}-1+\hat{b}\right) \beta_{1} \\
& =\gamma_{s}^{*}\left\{\left(i \omega_{0}-q_{-}^{0}\right) \frac{\beta_{0}}{T_{b}^{0}-1}-\frac{\beta_{0}+2 i \omega_{0}}{T_{m}-1}\left[q_{+}^{0} q_{+}^{0} \frac{T_{b}^{0}-T_{m}}{T_{b}^{0}-1}-\left(q_{-}^{0}+i \omega_{0}\right)\right] e^{\left(q_{-}^{0}-q_{+}^{0}\right)\left(x_{r}^{0}-x_{m}^{0}\right)_{o}}\right\} \\
& +\frac{\alpha_{s}}{1-\alpha_{s}}\left\{\left[\left(\hat{l}^{*}-\hat{r}^{*} \hat{b}\right) \ln \left(\frac{T_{b}^{0}-1}{T_{m}-1}\right)+\hat{r}^{*}\left(\chi p_{b} \frac{\hat{b} T_{b}^{0}-1+(1-\hat{b}) T_{m}}{\left(T_{m}-1\right)\left(T_{b}^{0}-1\right)}-1\right)\right]\right. \\
& \times\left[\left(\beta_{0}+2 i \omega_{0}\right) e^{\left(q_{-}^{0}-q_{+}^{0}\right)\left(x_{r}^{0}-x_{m}^{o}\right)_{0}}+\beta_{0} q_{-}^{0}\right] \\
& +\hat{r}^{*} \hat{b} \chi \frac{\beta_{0}}{T_{b}^{0}-1}\left(T_{m}-1\right) q_{-}^{0} \frac{i \omega_{0}}{i \omega_{0}+k^{2}} e^{q_{-}^{0}\left(x_{r}^{0}-x_{m}^{o}\right)_{0}} \\
& +\hat{r}^{*} \hat{b} \chi\left(\beta_{0}+2 i \omega_{0}\right) q_{+}^{o}\left(\frac{p_{b}-T_{m}+1}{T_{m}-1}-\frac{1}{2 q_{+}^{0}-1}\right) e^{\left(q_{-}^{o}-q_{+}^{o}\right)\left(x_{r}^{o}-x_{m}^{o}\right)_{0}} \\
& +\hat{r}^{*} \hat{b} \chi \beta_{0}\left[\frac{T_{b}^{0}-1}{T_{m}-1} \cdot \frac{\beta_{0}+2 i \omega_{0}}{\beta_{0}} e^{-q_{+}^{0}\left(x_{r}^{0}-x_{m}^{o}\right)_{0}}+i \omega_{0}\right] \frac{T_{m}\left(1-p_{b}\right)-1}{T_{m}\left(T_{b}^{0}-1\right)} e^{q_{-}^{0}\left(x_{r}^{0}-x_{m}^{0}\right)_{0}} \\
& +\left(\beta_{0}+2 i \omega_{0}\right)\left[\frac{\hat{r}^{*} \hat{b} q_{+}^{0}-\hat{l}^{*}\left(i \omega_{0}+q_{+}^{0}\right)}{2 q_{+}^{0}-1}+q_{+}^{0} \hat{l}^{*}\right]-\beta_{0}\left[q_{-}^{0} \frac{i \omega_{0} \hat{r}^{*} \hat{b}}{i \omega_{0}+k^{2}}+q_{-}^{0} \frac{\hat{r}^{*} \hat{b} \chi p_{b}}{T_{m}-1}+\hat{l}^{*}\right] \\
& +\beta_{0}\left(\hat{l}^{*}-\hat{r}^{*} \hat{b}\right)\left[1+q_{-}^{0}+q_{-}^{0} \ln \left(\frac{T_{b}^{0}-1}{T_{m}-1}\right)\right] \\
& \left.+i \omega_{0} \hat{r}^{*} \frac{T_{b}^{0}-1-\chi p_{b}}{T_{b}^{0}-1}\left[2 q_{+}^{0}-\frac{1-\hat{b}}{T_{b}^{0}-1}\left(\frac{2 Q_{0} \beta_{0}}{1-\hat{b}}+\beta_{0}\left(T_{b}^{0}+1\right)+2\left(T_{b}^{0}-1\right)\right)\right]\right\}
\end{aligned}
$$

where $\beta_{0}$ and $\omega_{0}$ were given by Eqs. (8.26) and (8.27), respectively.

\section{Analysis of the Dispersion Relation}

The dispersion relation given by Eq. (9.18) reflects the effects of non-zero porosity as well as the effects of confinement. A corresponding result was derived previously for the unconfined problem [9], and in fact the left-hand side of Eq. (9.18) above for $i \omega_{1}$ is identical to the left-hand side of Eq. (94) in that work. The right-hand sides differ, however, reflecting not only the effects of 
confinement (as indicated by the presence of the burned pressure $p_{b}$ in various terms), but also the result of replacing the constant-pressure condition of the previous study with a Darcy formulation in the solid/gas region, and treating the case of weak permeability. Although the effects of the latter are not expected to be large in the limit of small overpressures $p_{b} \rightarrow 1$, it is worth noting that there remain some differences between the two models even in that limit, since a constant pressure is only recovered from Darcy's law in the limit of large permeability. Thus, even in the limit of zero overpressures, the present model provides for a nontrivial pressure field in the solid/gas region.

To obtain the neutral stability correction, we set $\mathcal{R}\left\{i \omega_{1}\right\}=0$ and equate real and imaginary parts of Eq. (9.18) separately to zero. A coupled system of linear equations for $\beta_{1}$ and $\omega_{1}$ is thus obtained as

$$
\begin{aligned}
& a_{1,1} \omega_{1}+a_{1,2} \beta_{1}=\frac{\alpha_{s}}{1-\alpha_{s}} c_{1,1}+\gamma_{s}^{*} c_{1,2}, \\
& a_{2,1} \omega_{1}+a_{2,2} \beta_{1}=\frac{\alpha_{s}}{1-\alpha_{s}} c_{2,1}+\gamma_{s}^{*} c_{2,2}
\end{aligned}
$$

where

$$
\begin{gathered}
a_{1,1}=q_{i}+\frac{2 \omega_{0} q_{r}-\beta_{0} q_{i}}{q_{r}^{2}+q_{i}^{2}}, \quad a_{1,2}=-\frac{\omega_{0} q_{i}}{\beta_{0}} \\
a_{2,1}=\beta_{0}+1-2 \hat{b}-q_{r}-\frac{2 \omega_{0} q_{i}+\beta_{0} q_{r}}{q_{r}^{2}+q_{i}^{2}}, \quad a_{2,2}=\frac{\omega_{0}}{\beta_{0}}\left(q_{r}-1+2 \hat{b}\right),
\end{gathered}
$$

$$
\begin{aligned}
c_{1,1}= & A_{1} \frac{\beta_{0}}{2}\left(1-q_{r}\right)+A_{1} e^{-q_{r} \ln \Upsilon}\left[\beta_{0} \cos \left(q_{i} \ln \Upsilon\right)+2 \omega_{0} \sin \left(q_{i} \ln \Upsilon\right)\right] \\
+ & \frac{1}{2} A_{4} \beta_{0}\left(T_{b}^{0}-1\right) e^{-q_{r} \ln \Upsilon} \cos \left(\frac{q_{i}}{2} \ln \Upsilon\right)\left\{\frac{T_{m}-1}{T_{b}^{0}-1} \cdot \frac{\omega_{0}^{2}\left(1-q_{r}\right)+\omega_{0} k^{2}}{\omega_{0}^{2}+k^{4}} e^{\frac{1}{2}\left(1+q_{r}\right) \ln \Upsilon}\right. \\
& +\left[A_{6}\left(1+q_{r}-2 \frac{\omega_{0}}{\beta_{0}} q_{i}\right)-\frac{q_{r}\left(1+q_{r}\right)+q_{i}\left(q_{i}+2 \omega_{0} / \beta\right)}{q_{r}^{2}+q_{i}^{2}}+2 A_{3} \Upsilon\right] \cos \left(\frac{q_{i}}{2} \ln \Upsilon\right) \\
& \left.+\left[A_{6}\left(q_{i}+2 \frac{\omega_{0}}{\beta_{0}}\left(1+q_{r}\right)\right)+\frac{q_{i}-2\left(\omega_{0} / \beta_{0}\right)\left[q_{r}\left(1+q_{r}\right)+q_{i}^{2}\right]}{q_{r}^{2}+q_{i}^{2}}+4 A_{3} \Upsilon \frac{\omega_{0}}{\beta_{0}}\right] \sin \left(\frac{q_{i}}{2} \ln \Upsilon\right)\right\} \\
+ & \frac{1}{2} A_{4} \beta_{0}\left(T_{b}^{0}-1\right) e^{-q_{r} \ln \Upsilon} \sin \left(\frac{q_{i}}{2} \ln \Upsilon\right)\left\{\frac{T_{m}-1}{T_{b}^{0}-1} \cdot \frac{\omega_{0} k^{2}\left(1-q_{r}\right)-\omega_{0}^{2}}{\omega_{0}^{2}+k^{4}} e^{\frac{1}{2}\left(1+q_{r}\right) \ln \Upsilon}\right. \\
& +\left[A_{6}\left(q_{i}+2 \frac{\omega_{0}}{\beta_{0}}\left(1+q_{r}\right)\right)+\frac{q_{i}-2\left(\omega_{0} / \beta_{0}\right)\left[q_{r}\left(1+q_{r}\right)+q_{i}^{2}\right]}{q_{r}^{2}+q_{i}^{2}}+4 A_{3} \Upsilon \frac{\omega_{0}}{\beta_{0}}\right] \cos \left(\frac{q_{i}}{2} \ln \Upsilon\right) \\
& \left.-\left[A_{6}\left(1+q_{r}-2 \frac{\omega_{0}}{\beta_{0}} q_{i}\right)-\frac{q_{r}\left(1+q_{r}\right)+q_{i}\left(q_{i}+2 \omega_{0} / \beta_{0}\right)}{2}+2 A_{3} \Upsilon\right] \sin \left(\frac{q_{i}}{2} \ln \Upsilon\right)\right\} \\
+ & A_{3} A_{4} \omega_{0} \beta_{0}\left(T_{b}^{0}-1\right) \sin \left(\frac{q_{i}}{2} \ln \Upsilon\right) e^{\frac{1}{2}\left(1-q_{r}\right) \ln \Upsilon}+\hat{l}^{*}\left[\frac{1}{2} \beta_{0}\left(q_{r}-1\right)-\omega_{0} q_{i}\right] \\
+ & \frac{1}{q_{r}^{2}+q_{i}^{2}}\left\{\frac{\beta_{0}}{2}\left[A_{5} q_{r}\left(1+q_{r}\right)+q_{i}\left(A_{5}-2 \hat{l}^{*} \omega_{0}\right)\right]+\omega_{0}\left(A_{5} q_{i}+2 q_{r} \hat{l}^{*}\right)\right\}-A_{2} \hat{r}^{*} \omega_{0} q_{i} \\
& -\frac{\hat{r}^{*} \hat{b} \omega_{0} \beta_{0}}{2\left(\omega_{0}^{2}+k^{4}\right)}\left[\left(1+q_{r}\right) \omega_{0}+q_{i} k^{2}\right]-\frac{1}{2} A_{5} \beta_{0}\left[2+\left(1-q_{r}\right)(1+\ln \Upsilon)\right]-\frac{\hat{r}^{*} \hat{b} \chi p_{b}}{2\left(T_{m}-1\right)} \beta_{0}\left(1-q_{r}\right)
\end{aligned}
$$




$$
\begin{aligned}
c_{1,2}= & \frac{\beta_{0}\left(q_{r}-1\right)}{2\left(T_{b}^{0}-1\right)} \\
& +\left\{\omega_{0} q_{i}\left[\left(1+q_{r}\right) \Upsilon+1\right]-2 \omega_{0}^{2}-\frac{\beta_{0} \Upsilon}{4}\left[\left(1+q_{r}\right)^{2}-q_{i}^{2}\right]+\frac{\beta_{0}}{2}\left(1-q_{r}\right)\right\} \frac{\cos \left(q_{i} \ln \Upsilon\right)}{T_{m}-1} e^{-q_{r} \ln \Upsilon} \\
& -\left\{\frac{\omega_{0} \Upsilon}{2}\left[\left(1+q_{r}\right)^{2}-q_{i}^{2}\right]-\omega_{0}\left(\beta_{0}+1-q_{r}\right)+\frac{\beta_{0}}{2} q_{i}\left[\left(1+q_{r}\right) \Upsilon+1\right]\right\} \frac{\sin \left(q_{i} \ln \Upsilon\right)}{T_{m}-1} e^{-q_{r} \ln \Upsilon},
\end{aligned}
$$

$$
\begin{aligned}
& c_{2,1}=A_{1} \frac{\beta_{0}}{2} q_{i}+A_{1} e^{-q_{r} \ln \Upsilon}\left[2 \omega_{0} \cos \left(q_{i} \ln \Upsilon\right)-\beta_{0} \sin \left(q_{i} \ln \Upsilon\right)\right] \\
& +\frac{1}{2} A_{4} \beta_{0}\left(T_{b}^{0}-1\right) e^{-q_{r} \ln \Upsilon} \cos \left(\frac{q_{i}}{2} \ln \Upsilon\right)\left\{\frac{T_{m}-1}{T_{b}^{0}-1} \cdot \frac{\omega_{0} k^{2}\left(1-q_{r}\right)-\omega_{0}^{2}}{\omega_{0}^{2}+k^{4}} e^{\frac{1}{2}\left(1+q_{r}\right) \ln \Upsilon}\right. \\
& +\left[A_{6}\left(q_{i}+2 \frac{\omega_{0}}{\beta_{0}}\left(1+q_{r}\right)\right)+\frac{q_{i}-2\left(\omega_{0} / \beta_{0}\right)\left[q_{r}\left(1+q_{r}\right)+q_{i}^{2}\right]}{q_{r}^{2}+q_{i}^{2}}+4 A_{3} \Upsilon \frac{\omega_{0}}{\beta_{0}}\right] \cos \left(\frac{q_{i}}{2} \ln \Upsilon\right) \\
& \left.-\left[A_{6}\left(1+q_{r}-2 \frac{\omega_{0}}{\beta_{0}} q_{i}\right)-\frac{q_{r}\left(1+q_{r}\right)+q_{i}\left(q_{i}^{2}+2 \omega_{0} / \beta_{0}\right)}{q_{r}^{2}+q_{i}^{2}}+2 A_{3} \Upsilon\right] \sin \left(\frac{q_{i}}{2} \ln \Upsilon\right)\right\} \\
& -\frac{1}{2} A_{4} \beta_{0}\left(T_{b}^{0}-1\right) e^{-q_{r} \ln \Upsilon} \sin \left(\frac{q_{i}}{2} \ln \Upsilon\right)\left\{\frac{T_{m}-1}{T_{b}^{0}-1} \cdot \frac{\omega_{0} k^{2}+\omega_{0}^{2}\left(1-q_{r}\right)}{\omega_{0}^{2}+k^{4}} e^{\frac{1}{2}\left(1+q_{r}\right) \ln \Upsilon}\right. \\
& +\left[A_{6}\left(1+q_{r}-2 \frac{\omega_{0}}{\beta_{0}} q_{i}\right)-\frac{q_{r}\left(1+q_{r}\right)+q_{i}\left(q_{i}+2 \omega_{0} / \beta_{0}\right)}{q_{r}^{2}+q_{i}^{2}}+2 A_{3} \Upsilon\right] \cos \left(\frac{q_{i}}{2} \ln \Upsilon\right) \\
& \left.+\left[A_{6}\left(q_{i}+2 \frac{\omega_{0}}{\beta_{0}}\left(1+q_{r}\right)\right)+\frac{q_{i}-2\left(\omega_{0} / \beta_{0}\right)\left[q_{r}\left(1+q_{r}\right)+q_{i}^{2}\right]}{q_{r}^{2}+q_{i}^{2}}+4 A_{3} \Upsilon \frac{\omega_{0}}{\beta_{0}}\right] \sin \left(\frac{q_{i}}{2} \ln \Upsilon\right)\right\} \\
& +A_{3} A_{4} \omega_{0} \beta_{0}\left(T_{b}^{0}-1\right) \cos \left(\frac{q_{i}}{2} \ln \Upsilon\right) e^{\frac{1}{2}\left(1-q_{r}\right) \ln \Upsilon}+\hat{l}^{*}\left[\frac{1}{2} \beta_{0} q_{i}+\omega_{0}\left(1+q_{r}\right)\right] \\
& +\frac{1}{q_{r}^{2}+q_{i}^{2}}\left\{-\frac{\beta_{0}}{2}\left(2 q_{r} \hat{l}^{*} \omega_{0}+A_{5} q_{i}\right)+\omega_{0}\left[A_{5} q_{r}\left(1+q_{r}\right)+q_{i}\left(A_{5} q_{i}-2 \hat{l}^{*} \omega_{0}\right)\right]\right\} \\
& +\frac{\hat{r}^{*} \hat{b} \omega_{0} \beta_{0}}{2\left(\omega_{0}^{2}+k^{4}\right)}\left[q_{i} \omega_{0}-\left(1-q_{r}\right) k^{2}\right]-\frac{1}{2} \beta_{0} q_{i}\left[A_{5}(1+\ln \Upsilon)+\frac{\hat{r}^{*} \hat{b} \chi p_{b}}{T_{m}-1}\right] \\
& +\hat{r}^{*} \omega_{0} A_{2}\left[q_{r}-1+2 \hat{b}-\beta_{0} \frac{2 Q_{0}+(1-\hat{b})\left(T_{b}^{0}+1\right)}{T_{b}^{0}-1}\right]
\end{aligned}
$$

$$
\begin{aligned}
c_{2,2}= & \beta_{0} \frac{q_{i}+2 \omega_{0}}{2\left(T_{b}^{0}-1\right)} \\
& -\left\{\frac{\beta_{0}}{2} q_{i}\left[\left(1+q_{r}\right) \Upsilon+1\right]-\beta_{0} \omega_{0}+\frac{\omega_{0} \Upsilon}{2}\left[\left(1+q_{r}\right)^{2}-q_{i}^{2}\right]-\omega_{0}\left(1-q_{r}\right)\right\} \frac{\cos \left(q_{i} \ln \Upsilon\right)}{T_{m}-1} e^{-q_{r} \ln \Upsilon} \\
& +\left(\frac{\beta_{0} \Upsilon}{4}\left[\left(1+q_{r}\right)^{2}-q_{i}^{2}\right]-\frac{\beta_{0}}{2}\left(1-q_{r}\right)-\omega_{0} q_{i}\left[\left(1+q_{r}\right) \Upsilon+1\right]+2 \omega_{0}^{2}\right\} \frac{\sin \left(q_{i} \ln \Upsilon\right)}{T_{m}-1} e^{-q_{r} \ln \Upsilon}
\end{aligned}
$$

Here, $\Upsilon, q_{r}$ and $q_{i}$ are defined as

$$
\Upsilon=\frac{T_{b}^{0}-1}{T_{m}-1}, \quad \sqrt{1+4\left(i \omega_{0}+k^{2}\right)} \equiv q_{r}+i q_{i}
$$


or, equivalently,

$$
\left\{\begin{array}{l}
q_{r} \\
q_{i}
\end{array}\right\}=\frac{\sqrt{2}}{2} \sqrt{\sqrt{\left(1+4 k^{2}\right)^{2}+16 \omega_{0}^{2}} \pm\left(1+4 k^{2}\right)},
$$

where the principal root has been taken in the definition (10.8), and

$$
\begin{gathered}
A_{1}=-A_{5} \ln \Upsilon+\hat{r}^{*}\left[\chi p_{b} \frac{\hat{b} T_{b}^{0}-1+(1-\hat{b}) T_{m}}{\left(T_{m}-1\right)\left(T_{b}^{0}-1\right)}-1\right], \quad A_{2}=\frac{T_{b}^{0}-1-\chi p_{b}}{T_{b}^{0}-1}, \\
A_{3}=\frac{T_{m}\left(1-p_{b}\right)-1}{T_{m}}, \quad A_{4}=\frac{\hat{r}^{*} \hat{b} \chi}{T_{b}^{0}-1}, \quad A_{5}=\hat{r}^{*} \hat{b}-\hat{l}^{*}, \quad A_{6}=\frac{p_{b}-T_{m}+1}{T_{m}-1} .
\end{gathered}
$$

Equation (10.1) is easily solved to obtain

$$
\beta_{1}=\frac{\alpha_{s}}{1-\alpha_{s}} \hat{\beta}_{\alpha}+\gamma_{s}^{*} \hat{\beta}_{\gamma}
$$

where

$$
\hat{\beta}_{\alpha}=\frac{a_{1,1} c_{2,1}-a_{2,1} c_{1,1}}{a_{1,1} a_{2,2}-a_{2,1} a_{1,2}}, \quad \hat{\beta}_{\gamma}=\frac{a_{1,1} c_{2,2}-a_{2,1} c_{1,2}}{a_{1,1} a_{2,2}-a_{2,1} a_{1,2}} .
$$

We observe from Eqs. (10.12) that the perturbation coefficient $\beta_{1}$ consists of two contributions, where the first isolates the dependence on the porosity $\alpha_{s}$ and the second accounts for all effects associated with the heat of melting $\gamma_{s}^{*}$. The coefficients $\hat{\beta}_{\alpha}$ and $\hat{\beta}_{\gamma}$ are independent of these parameters, but the first of these is inherently coupled with the effects of two-phase flow through parametric dependencies on the gas-to-solid density, thermal-conductivity and heat-capacity ratios $\hat{r}^{*}, \hat{l}^{*}$ and $\hat{b}$, respectively, and the burned overpressure $p_{b}$. The last of these, which reflects the effects of confinement and the resulting preheating of the solid through pressure-driven permeation of the hot gas into the porous solid, is the main parameter of interest here. The other parametric influences on the location of the neutral stability boundary have been studied in detail in connection with the unconfined problem $[8,9]$. We thus focus on illustrating the variation of the stability boundary with the overpressure $p_{b}$, noting that all changes associated with confinement are scaled by the factor $\alpha_{s}\left(1-\alpha_{s}\right)^{-1}$.

Before proceeding to determine a correction to the leading-order stability boundary given by Eqs. (8.26) and (8.27), it is useful to define an alternative stability parameter first. We thus define, as in $[8,9]$, a modified Zel'dovich number $\beta^{0}$ that, unlike the present parameter $\beta$ defined by Eqs. (4.1) and (3.2), is independent of the precise value of $T_{b}$, which, according to Eq. (3.15), varies with a number of other parameters, including the overpressure $p_{b}$. In particular, noting the expansion $T_{b} \sim T_{b}^{0}+\epsilon T_{b}^{1}+\cdots$ introduced in Eq. (7.1c), we calculate from Eq. (3.15) and the scalings (7.1a) the expression given in Eq. (8.23) for $T_{b}^{0}$, and the next-order correction $T_{b}^{1}=-\hat{r}^{*} \alpha_{s}\left(1-\alpha_{s}\right)^{-1}\left[T_{b}^{0}-\left(1+\chi p_{b}\right)\right]$. We now introduce a modified Zel'dovich number based only on $T_{b}^{0}$ as

$$
\beta^{0}=\left[1-\left(T_{b}^{0}\right)^{-1}\right] N^{0}, \quad N^{0}=\frac{\tilde{E}}{\tilde{R}^{\circ} \tilde{T}_{b}^{0}}=\frac{\tilde{E}}{\tilde{R}^{\circ} \tilde{T}_{u} T_{b}^{0}}
$$


and thus the relationship between $\beta \sim \beta_{0}+\epsilon \beta_{1}+\cdots$ and $\beta^{0} \sim \beta_{0}^{0}+\epsilon \beta_{1}^{0}+\cdots$ is given by

$$
\beta_{0}^{0}=\beta_{0}, \quad \beta_{1}^{0}=\beta_{1}+\hat{\beta}_{1}, \quad \hat{\beta}_{1}=-\hat{r}^{*} \frac{\alpha_{s}}{1-\alpha_{s}}\left(\beta_{0}-\frac{\chi p_{b} N^{0}}{T_{b}^{0}}\right) \frac{T_{b}^{0}-2}{T_{b}^{0}} .
$$

Consequently, the total expression through $O(\epsilon)$ for the neutral stability boundary with respect to the modified parameter $\beta^{0}$ is given by

$$
\beta^{0} \sim \beta_{0}+\left\{\frac{\alpha_{s}}{1-\alpha_{s}}\left[\hat{\beta}_{\alpha}-\hat{r}^{*}\left(\beta_{0}-\frac{\chi p_{b} N^{0}}{T_{b}^{0}}\right) \frac{T_{b}^{0}-2}{T_{b}^{0}}\right]+\hat{\beta}_{\gamma} \gamma_{s}^{*}\right\} \epsilon+\cdots
$$

From Eq. (10.15), it is seen that the correction to the leading-order neutral stability boundary $\beta_{0}(k)$ is composed of three contributions; a term proportional to $\alpha_{s} \hat{\beta}_{\alpha} /\left(1-\alpha_{s}\right)$ that reflects the influence of porosity and two-phase transport, a term proportional to $\gamma_{s}^{*} \hat{\beta}_{\gamma}$ that contains those effects arising from the endothermicity or exothermicity associated with melting, and a term proportional to $\alpha_{s} \hat{\beta}_{1} /\left(1-\alpha_{s}\right)$ that stems from the change in the stability parameter itself as other parameters that affect the combustion temperature are varied. It can be seen from the appearance of $p_{b}$ in the expressions for the coefficients in Eqs. (10.1) that the value of the overpressure influences the stability boundary through $\hat{\beta}_{\alpha}$ and $\hat{\beta}_{1}$, but not through $\hat{\beta}_{\gamma}$. Consequently, since the focus here is on influences associated with confinement, we shall neglect heat-of-melting effects, which have already been discussed in the context of the unconfined problem $[8,9]$, by setting $\gamma_{s}^{*}$ to zero.

Plots of the leading-order stability boundaries $\beta_{0}(k)$ and the modified boundaries $\beta^{0}(k)$, given by Eq. (10.15) through $O(\epsilon)$, are shown in Figures $2 \mathrm{a}-\mathrm{f}$ for a representative value of $\epsilon$ and various values of the gas-to-solid heat-capacity ratio $\hat{b}$, thermal-conductivity ratio $\hat{l}^{*}$ and overpressure $p_{b}$. The relatively large shifts in the leading-order boundary $\beta_{0}(k)$ partly reflect the $O(1)$ change in the definition of the stability parameter $\beta_{0}$ with $\hat{b}$ through its primarily inverse dependence on the leading-order combustion temperature $T_{b}^{0}$, which in turn is inversely proportional to $\hat{b}$ according to Eq. (8.23). In other words, the Zel'dovich number roughly scales with $\hat{b}$, so that much of the shift in the stability boundary with $\hat{b}$ is accounted for by the change in the definition of $\beta_{0}$ as that parameter is varied. Nonetheless, it is clear that higher combustion temperatures associated with lower gas-phase heat capacities give rise to instabilities occurring at smaller Zel'dovich numbers. The expression for $\beta_{0}(k)$, given by Eq. (8.26), is independent of $p_{b}$, and it is thus the modifications to this boundary for fixed values of $\hat{b}$ that determine the influences of confinement on the critical Zel'dovich number $\beta^{0}$ as a function of disturbance wavenumber $k$.

The most obvious trend regarding the corrections to the leading-order stability boundary in Figures $2 a-f$ is the stabilizing effect associated with increasing overpressure $p_{b}$. As this parameter increases, the initial correction for $p_{b}=0$, which may be positive or negative, always moves the modified boundary in the positive direction, thereby enhancing the parameter range in which steady, planar burning is stable. This trend is primarily attributed to the slowing down and eventual reversal of the gas flow in the direction of the unburned solid, resulting in an increase in burned 
temperature and reduction in the effective Zel'dovich number that accompanies the gas permeation and preheating of the material. Thus, in Figures $2 a-d$, which correspond to different values of $\hat{b}$, the initial two-phase-flow effects for the unconfined limit $p_{b}=0$ shift from stabilizing to destabilizing as the gas-to-solid heat-capacity ratio $\hat{b}$ increases (and hence $T_{b}^{0}$ decreases), but the increasing convective influence of the reverse gas flow in the gas-permeation layer that accompanies an increase in the overpressure tends to result in an overall two-phase-flow influence that is always stabilizing for sufficiently large values of $p_{b}$. Consistent with this argument, we note that the stabilizing influence of nonzero overpressures thus tends to be more pronounced for small wavenumbers (large wavelengths), suggesting that longitudinal perturbations normal to the basic planar solution are more significantly dampened by this effect than are the transverse (cellular) components of the disturbance.

The gas-to-solid thermal-conductivity ratio $\hat{l}^{*}$, which was held constant in Figures $2 a-d$, is decreased in Figures 2e and $2 \mathrm{f}$ relative to the values used in Figures $2 \mathrm{a}$ and 2c, respectively. By comparing these two sets of curves for different values of $\hat{l}^{*}$, it is seen that the effect of decreasing the conductivity ratio is significantly destabilizing. This result is logically attributed to the fact that reduced gas-phase heat transfer is less able to dampen transverse disturbances and to participate, along with convection, in the stabilizing transport of heat into the virgin porous solid. The combined effects of changes in $\hat{b}$ and $\hat{l}^{*}$ on the corrections to the leading-order boundary may be synthesized by noting, based on the above discussion, that increases in the gas-to-solid diffusivity ratio (proportional to $\hat{l}^{*} / \hat{b}$ ) are stabilizing.

Figures $2 a-f$ represent the combined effects of the terms proportional to $\hat{\beta}_{\alpha}$ and $\hat{\beta}_{1}$ in Eq. (10.15), where, as indicated by Eqs. (10.13) and (10.14), $\epsilon \hat{\beta}_{1}$ accounts for that portion of the shift in the stability boundary arising from the variation of the original Zel'dovich number $\beta$ with the combustion temperature $T_{b}$, which varies with $p_{b}=p_{g}^{b}-1$ according to Eq. (3.15). It is clear that the magnitude of the stabilizing or destabilizing effects just described are proportional to $\alpha_{s} /\left(1-\alpha_{s}\right)$ and thus become greater as the porosity, which is responsible for two-phase-flow effects, increases. Whereas the parametric influences on $\hat{\beta}_{1}$ are clear from the last of Eqs. (10.14), the behavior of the contribution arising from $\hat{\beta}_{\alpha}$ is, however, much less explicit. Accordingly, we plot in Figures $3 \mathrm{a}, \mathrm{b}$ the contribution $\beta_{1}=\alpha_{s} \hat{\beta}_{\alpha} /\left(1-\alpha_{s}\right.$ ) (for $\gamma_{s}^{*}=0$ ) along with the combined result $\beta_{1}+\hat{\beta}_{1}$ that accounts for the total correction in Figures $2 \mathrm{a}-\mathrm{f}$. It can be seen from Figures $3 \mathrm{a}$ and $3 \mathrm{~b}$ that the stabilizing effects of nonzero overpressures is mainly attributable to $\beta_{1}$, since, according to Eq. (10.14), the contribution from $\hat{\beta}_{1}$, starts out negative and, for a given value of $k$, only becomes positive for sufficiently large overpressures given by $p_{b}>T_{b}^{0} \beta_{0}(k) / \chi N^{0}$. It is also seen that the stabilizing influence of $\beta_{1}$ is weakest at intermediate disturbance wavenumbers. For both large and small wavenumbers, the stabilizing influences of thermal diffusivity in dampening transverse and longitudinal components, respectively, of the perturbation become increasingly significant. Hence, $\beta_{1}(k)$ typically possesses a minimum at some nonzero value of $k$. The curves corresponding to 
the sum $\beta_{1}+\hat{\beta}_{1}$ in Figures $3 a, b$, when multiplied by $\epsilon$, give the difference between the corrected and leading-order boundaries in Figures 2a and 2c, respectively. Again, the results emphasize that the effects of nonzero overpressures associated with confinement are stabilizing with respect to the leading-order (unconfined) stability boundary.

\section{Conclusions}

The present work has extended previous stability analyses of unconfined deflagrations in porous energetic materials to include the effects of confinement. Assuming gas-phase quasi-steadiness, confinement was effectively modeled by allowing for a nonzero overpressure in the burned-gas region with respect to the gas in the unburned solid. This overpressure, which tends to reverse the gas flow, leads to a permeation of the hot gas into the unburned porous solid and a consequent preheating of the material through convective heat transfer. This preheating, in turn, results in a superadiabatic effect that increases the combustion temperature (and wave speed) of the basic solution corresponding to steady, planar burning, and produces a shift in the stability boundaries delineating the growth or decay of nonsteady, nonplanar perturbations arising from thermal/diffusive instability.

An analysis of the basic solution was previously given based on a perturbation analysis of an asymptotic model, derived in the limit of large overall activation energy, that treated two overpressure regimes of interest, including the boundary-layer limit of the gas-permeation region considered here. This limit is sufficient to describe the onset of convective burning for weak permeabilities and moderate overpressures, although for larger magnitude overpressures associated with fully-developed convective flow, the gas-permeation layer extends deeper into the solid-gas region [12]. To facilitate a stability analysis of the basic solution in the boundary-layer regime, a nonsteady, multidimensional version of the asymptotic model was described, and an earlier assumption of small porosity was relaxed. A perturbation analysis, based on the realistic smallness of the permeability, heat of melting and gas-to-solid density and thermal-conductivity ratios, was then developed to the necessary order, both for the basic solution and the nonsteady, nonplanar perturbation variables. A significant aspect of the calculation, relative to that for the unconfined problem, was the development of inner and outer solutions with respect to the gas-permeation layer, and the matching of these solutions to the order necessary to determine leading- and firstorder corrections to the dispersion relation. Analysis of the latter then determined the effects of confinement (overpressure) and porosity on the stability boundaries relative to the unconfined and/or nonporous problems.

As in the unconfined problem, the effects of porosity and two-phase flow on the shift in stability boundaries relative to the nonporous case are essentially proportional to $\alpha_{s} /\left(1-\alpha_{s}\right)$, where $\alpha_{s}$ is the initial gas-phase volume fraction in the solid/gas region. The modifications arising from 
confinement are then reflected in the explicit presence of the overpressure parameter $p_{b}$, equal to the pressure difference across the combustion wave, in various terms of the final dispersion relation that governs the onset of the present thermal/diffusive type of instability. It was found that the primary effects arising from confinement are stabilizing relative to the stability boundaries obtained for the unconfined problem, resulting in a widening of the region of stability for steady, planar combustion. This stabilizing influence, which increases as $p_{b}$ increases, is largely attributed to the pressure-driven convective preheating of the material and rise in combustion temperature, which lowers the effective nondimensional activation energy. This type of superadiabatic effect is thus analogous to the stabilizing effects of decreasing heat losses and increasing combustion temperature on stability in nonadiabatic single-phase deflagrations. Other two-phase-flow effects that enhance thermal/diffusive stability relative to the leading-order (nonporous) stability boundary include those associated with increasing the gas-to-solid thermal diffusivity ratio, which, as in the unconfined case, contributes to the dampening of transverse temperature disturbances. In the confined case of interest here, thermal diffusivity also acts in conjunction with the stabilizing convective transport of heat into the unburned material, thereby enhancing the convective preheating of the unburned material through diffusion of heat from the burned to the unburned region.

In addition to the aforementioned aspects of confined deflagrations in porous materials, the present theory contains effects associated with other parameters that could be explored more fully in other work. It would also be of interest to study the complete transition to fully convective burning and its associated instabilities, which could be quite different from the results in the transition region presented here.

\section{Acknowledgement}

This work was partly supported by the U. S. Department of Energy under Contract DE-AC0494AL85000.

\section{References}

1. Kuo, K. K., and Summerfield, M., "Theory of Steady-State Burning of Gas-Permeable Propellants," AIAA Journal, Vol. 12, 1974, pp. 49-56.

2. Ermolayev, B. S., Borisov, A. A., and Khasainov, B. A., "Comments on "Theory of SteadyState Burning of Gas-Permeable Propellants,' " AIAA Journal, Vol. 13, 1975, p. 1128.

3. Gokhale, S. S., and Krier, H., "Modeling of Unsteady Two-Phase Reactive Flow in Porous Beds of Propellant," Progress in Energy and Combustion Science, Vol. 8, 1982, pp. 1-39.

4. Margolis, S. B., Williams, F. A. and Armstrong, R. C. "Influences of Two-Phase Flow in the Deflagration of Homogeneous Solids," Combustion and Flame, Vol. 67, 1987, pp. 249-258. 
5. Li, S. C., Williams, F. A., and Margolis, S. B., "Effects of Two-Phase Flow in a Model for Nitramine Deflagration," Combustion and Flame, Vol. 80, 1990, pp. 329-349.

6. Asay, B. W., Son, S. F., and Bdzil, J. B., "The Role of Gas Permeation in Convective Burning," International Journal of Multiphase Flow, Vol. 22, 1996, pp. 923-952.

7. Margolis, S. B., and Williams, F. A., "Effects of Two-Phase Flow on the Deflagration of Porous Energetic Materials," Journal of Propulsion and Power, Vol. 11, 1995, pp. 759-768.

8. Margolis, S. B., and Williams, F. A., "Influence of Porosity and Two-Phase Flow on Diffusional/Thermal Instability of a Deflagrating Energetic Material," Combustion Science and Technology, Vol. 106, 1995, pp. 41-68.

9. Margolis, S. B., and Williams, F. A., "Effect of Gas-Phase Thermal Expansion on Stability of Deflagrations in Porous Energetic Materials," International Journal of Multiphase Flow, Vol. 22, 1996, pp. 69-91.

10. Margolis, S. B., "A Deflagration Analysis of Porous Energetic Materials with Two-Phase Flow and a Multiphase Reaction Mechanism," Journal of Engineering Mathematics, Vol. 31, 1997, pp. 173-203.

11. Ilincic, N., and Margolis, S. B., "Eigenvalue Analysis and Calculations for the Deflagration of Porous Energetic Materials in the Merged-Flame Regime," Combustion Science and Technology, Vol. 125, 1997, pp. 201-241.

12. Margolis, S. B., "Influence of Pressure-Driven Gas Permeation on the Quasi-Steady Burning of Porous Energetic Materials," Combustion Theory and Modelling, Vol. 2, 1998, pp. 95-113.

13. Baer, M. R. and Nunziato, J. W., "A Two-Phase Mixture Theory for the Deflagration-toDetonation Transition (DDT) in Reactive Granular Materials," International Journal of Multiphase Flow, Vol. 12, 1986, pp. 861-889.

14. Boggs, T. L., "The Thermal Behavior of Cyclotrimethylenetrinitramine (RDX) and Cyclotetramethylenetetranitramine (HMX)," edited by K. K. Kuo and M. Summerfield, Vol. 90, Progress in Astronautics and Aeronautics, AIAA, New York, 1984, pp. 121-175.

15. Baer, M. R., and Shepherd, J. E., "A Thin Flame Model for Reactive Flow in Porous Materials," Sandia National Laboratories, SAND83-2576, Albuquerque, NM, 1984.

16. Margolis, S. B., and Williams, F. A., "Structure and Stability of Deflagrations in Porous Energetic Materials," Journal of Propulsion and Power, 1999, to appear.

17. Denison, M. R., and Baum, E., "A Simplified Model of Unstable Burning in Solid Propellants," ARS Journal, Vol. 31, 1961, pp. 1112-1122.

18. De Luca, L., "Theory of nonsteady burning and combustion stability of solid propellants by flame models," in Nonsteady Burning and Combustion Stability of Solid Propellants (Edited by De Luca, L., Price, E. W. \& Summerfield, M.), Progress in Astronautics and Aeronautics, 
Vol. 143, 1992, pp. 519-600.

19. Margolis, S. B., and Williams, F. A., "Diffusional/Thermal Coupling and Intrinsic Instability of Solid Propellant Combustion," Combustion Science and Technology, Vol. 59, 1988, pp. $27-84$.

20. Margolis, S. B., and Williams, F. A., "Diffusional/Thermal Instability of a Solid Propellant Flame," SIAM Journal on Applied Mathematics, Vol. 49, 1989, pp. 1390-1420.

21. Nayfeh, A. H., Perturbation Methods, (John Wiley \& Sons). 1973. 


\section{APPENDIX A. Analysis of the Basic Solution in the Gas-Permeation Sublayer}

The expansion coefficients of the basic solution in both the outer and inner portions of the solid/gas region appear in the corresponding equations for the linear stability variables in Sections 8 and 9. Accordingly, we present an analysis of the basic problem for overpressure, which is complicated by the fact that there is a boundary layer adjacent to the melting surface. Following the approach in [12], we introduce the scalings (7.1a) into the pressure equation (5.11b) and the corresponding boundary conditions, and seek inner and outer solutions as expansions in $\epsilon$.

Within the boundary layer, a solution for the overpressure variable $p$ is sought in the form $p=p_{0}+\epsilon p_{1}+\epsilon^{2} p_{2}+\cdots$. Substituting this expansion and the parameter scalings into Eq. (5.11b) written in the boundary-layer coordinate $\hat{\eta}$ we obtain

$$
\begin{gathered}
\left(1-\alpha_{s}+\epsilon \hat{r}^{*} \hat{b} \alpha_{s}\right)\left[p_{0}+\epsilon p_{1}+\cdots-\frac{\kappa^{*}}{\alpha_{s}}\left(p_{0}+1+\epsilon p_{1}+\cdots\right) \frac{d}{d \hat{\eta}}\left(p_{0}+\epsilon p_{1}+\cdots\right)\right] \\
=\left(1-\alpha_{s}+\epsilon \hat{l}^{*} \alpha_{s}\right) \epsilon^{-1} \frac{d}{d \hat{\eta}}\left[p_{0}+\epsilon p_{1}+\cdots-\frac{\kappa^{*}}{\alpha_{s}}\left(p_{0}+1+\epsilon p_{1}+\cdots\right) \frac{d}{d \hat{\eta}}\left(p_{0}+\epsilon p_{1}+\cdots\right)\right] \\
+\epsilon \hat{r}^{*} \hat{b} \chi \alpha_{s}\left(p_{0}+\epsilon p_{1}+\cdots\right),
\end{gathered}
$$

subject to

$$
p_{0}+\epsilon p_{1}+\ldots=p_{b}, \quad \epsilon^{-1} \frac{d}{d \hat{\eta}}\left(p_{0}+\epsilon p_{1}+\cdots\right)=\frac{\alpha_{s}}{\kappa^{*}} \epsilon^{-1}\left(\frac{p_{b}-T_{m}+1}{p_{b}+1}\right) \text { at } \hat{\eta}=0 .
$$

We note that the value of $\alpha_{s}$ is here assumed $O(1)$, whereas in the previous analysis [12] this parameter was assumed to be $O(\epsilon)$.

At leading order, the problem (A.1) - (A.2) simplifies to

$$
\begin{gathered}
\frac{d}{d \hat{\eta}}\left[p_{0}-\frac{\kappa^{*}}{\alpha_{s}}\left(p_{0}+1\right) \frac{d p_{0}}{d \hat{\eta}}\right]=0, \\
p_{0}=p_{b}, \quad \frac{d p_{0}}{d \hat{\eta}}=\frac{\alpha_{s}}{\kappa^{*}}\left(\frac{p_{b}-T_{m}+1}{p_{b}+1}\right) \text { at } \hat{\eta}=0 .
\end{gathered}
$$

By integrating Eq. (A.3) twice and applying the boundary conditions (A.4), an implicit solution for the leading-order overpressure $p_{0}$ is obtained as

$$
\frac{\alpha_{s}}{\kappa^{*}} \hat{\eta}=p_{0}-p_{b}+T_{m} \ln \left(\frac{p_{0}-T_{m}+1}{p_{b}-T_{m}+1}\right) .
$$

To determine $p_{1}$, we consider the next-order problem with respect to $\epsilon$, obtaining from Eqs. (A.1) - (A.2) the problem

$$
\begin{gathered}
\left(1-\alpha_{s}\right)\left[p_{0}-\frac{\kappa^{*}}{\alpha_{s}}\left(p_{0}+1\right) \frac{d p_{0}}{d \hat{\eta}}\right]=\left(1-\alpha_{s}\right) \frac{d}{d \hat{\eta}}\left[p_{1}-\frac{\kappa^{*}}{\alpha_{s}}\left(p_{0}+1\right) \frac{d p_{1}}{d \hat{\eta}}-\frac{\kappa^{*}}{\alpha_{s}} p_{1} \frac{d p_{0}}{d \hat{\eta}}\right] \\
+\hat{l}^{*} \alpha_{s} \frac{d}{d \hat{\eta}}\left[p_{0}-\frac{\kappa^{*}}{\alpha_{s}}\left(p_{0}+1\right) \frac{d p_{0}}{d \hat{\eta}}\right],
\end{gathered}
$$




$$
p_{1}=0, \quad \frac{d p_{1}}{d \hat{\eta}}=0 \text { at } \hat{\eta}=0
$$

Since $p_{0}(\hat{\eta})$ is given implicitly by Eq. (A.5), we may solve Eqs. (A.6) - (A.7) for $p_{1}$ as a function of $p_{0}$. Using the previous results (A.3) - (A.4), the solution for $p_{1}\left(p_{0}\right)$ is obtained as

$$
\begin{aligned}
& p_{1}=\left(T_{m}-1\right) \frac{\kappa^{*}}{\alpha_{s}}\left(\frac{p_{0}-T_{m}+1}{p_{b}-T_{m}+1}\right)\left\{\left(p_{b}-p_{0}\right)\left[1-T_{m} \frac{p_{b}-2 T_{m}+1}{\left(p_{0}-T_{m}+1\right)\left(p_{b}-T_{m}+1\right)}\right]\right. \\
& \left.+\left(p_{b}-2 T_{m}+1\right) \ln \left(\frac{p_{0}-T_{m}+1}{p_{b}-T_{m}+1}\right)-\frac{T_{m}}{2} \ln ^{2}\left(\frac{p_{0}-T_{m}+1}{p_{b}-T_{m}+1}\right)+\frac{T_{m}^{2}}{p_{0}-T_{m}+1}\left(\frac{p_{0}-T_{m}+1}{p_{b}-T_{m}+1}\right)\right\} .
\end{aligned}
$$

The temperature and the pressure are related in the gas-permeation layer according to Darcy's law. Thus, in the boundary layer we expand the result (5.11a) as

$$
\hat{T}_{0}+\epsilon \hat{T}_{1}+\cdots=1+p_{0}+\epsilon p_{1}+\cdots-\frac{\kappa^{*}}{\alpha_{s}}\left(1+p_{0}+\epsilon p_{1}+\cdots\right) \frac{d}{d \hat{\eta}}\left(p_{0}+\epsilon p_{1}+\cdots\right),
$$

where the left-hand side is the expansion for the basic temperature in the boundary layer. Thus, each temperature coefficient and its spatial derivatives can be expressed in terms of the leadingorder overpressure. One can continue with this procedure to obtain equations and solutions for the $p_{2}, p_{3}$ and so forth. However, based on the jump conditions across the melting surface, it is sufficient to have solutions for $p_{0}$ and $p_{1}$ in the boundary layer in order to ultimately obtain the dispersion relation to $\mathrm{O}(\epsilon)$. As for the spatial derivatives of the basic temperature that are needed in the boundary layer, Eq. (A.9) indicates that they can be readily obtained by differentiating the pressure equations with respect to $\hat{\eta}$. Hence, the higher-order versions of Eqs. (A.1) - (A.2) can be differentiated with respect to $\hat{\eta}$ to ultimately yield $d T_{0} / d \hat{\eta}, d T_{1} / d \hat{\eta}, d^{2} T_{1} / d \hat{\eta}^{2}, d T_{2} / d \hat{\eta}, d^{2} T_{2} / d \hat{\eta}^{2}$ and $d^{2} T_{3} / d \hat{\eta}^{2}$, all of which appear at some point in the perturbation analysis described in Sections 8 and 9 .

Turning our attention now to the outer problem, we observe that the overpressure outside the boundary layer within the solid/gas region, denoted by $q$, is to be expanded according to Eq. (7.1e). Thus, the corresponding sequence of pressure problems outside the boundary layer is determined from the expansion of Eq. (5.11b) according to

$$
\begin{aligned}
& \left(1-\alpha_{s}+\epsilon \hat{r}^{*} \hat{b} \alpha_{s}\right)\left[q_{0}+\epsilon q_{1}+\cdots-\epsilon \frac{\kappa^{*}}{\alpha_{s}}\left(q_{0}+1+\epsilon q_{1}+\cdots\right) \frac{d}{d \zeta}\left(q_{0}+\epsilon q_{1}+\cdots\right)\right] \\
& =\left(1-\alpha_{s}+\epsilon \hat{l}^{*} \alpha_{s}\right) \frac{d}{d \zeta}\left[q_{0}+\epsilon q_{1}+\cdots-\epsilon \frac{\kappa^{*}}{\alpha_{s}}\left(q_{0}+1+\epsilon q_{1}+\cdots\right) \frac{d}{d \zeta}\left(q_{0}+\epsilon q_{1}+\cdots\right)\right] \\
& +\epsilon \hat{r}^{*} \hat{b} \chi \alpha_{s}\left(q_{0}+\epsilon q_{1}+\cdots\right) \text {, } \\
& q_{0}+\epsilon q_{1}+\cdots=0 \text { as } \zeta \rightarrow-\infty .
\end{aligned}
$$

At leading order, the equation for $q_{0}$ is given simply by

$$
q_{0}=\frac{d q_{0}}{d \zeta}
$$


which has the admissible solution $q_{0}=a_{1} \exp (\zeta)$. Since the boundary-layer (inner) solution $p_{0} \rightarrow$ $\left(T_{m}-1\right)$ as $\hat{\eta} \rightarrow-\infty$, whereas the outer solution $q_{0} \rightarrow a_{1} e^{-\left(x_{r}^{0}-x_{m}^{0}\right)_{o}}$ as $\zeta \rightarrow-\left(x_{r}^{0}-x_{m}^{0}\right)$, the leading-order matching of these solutions implies $a_{1}=\left(T_{m}-1\right) e^{\left(x_{r}^{0}-x_{m}^{0}\right)_{0}}$. Hence,

$$
q_{0}=\left(T_{m}-1\right) e^{\zeta+\left(x_{r}^{0}-x_{m}^{\circ}\right)_{0}}
$$

At the next order, Eq. (A.10) yields an equation for the coefficient $q_{1}$ given by

$$
\left(1-\alpha_{s}\right)\left(q_{1}-\frac{d q_{1}}{d \zeta}\right)=\hat{l}^{*} \alpha_{s} \frac{d q_{0}}{d \zeta}+\left(1-\alpha_{s}\right) \frac{\kappa^{*}}{\alpha_{s}}\left\{\left(q_{0}+1\right) \frac{d q_{0}}{d \zeta}-\frac{d}{d \zeta}\left[\left(q_{0}+1\right) \frac{d q_{0}}{d \zeta}\right]\right\}+\hat{r}^{*} \hat{b} \alpha_{s}(\chi-1) q_{0} .
$$

Using the leading-order result (A.12) for $q_{0}$, Eq. (A.14) becomes

$$
\frac{d q_{1}}{d \zeta}-q_{1}=\frac{\kappa^{*}}{\alpha_{s}}\left(T_{m}-1\right)^{2} e^{2\left[\zeta+\left(x_{r}^{0}-x_{m}^{0}\right)_{0}\right]}-\frac{\alpha_{s}}{1-\alpha_{s}}\left[\hat{l}^{*}-\hat{r}^{*} \hat{b}(1-\chi)\right]\left(T_{m}-1\right) e^{\zeta+\left(x_{r}^{0}-x_{m}^{0}\right)_{0}},
$$

which has the solution

$$
\begin{aligned}
q_{1}=e^{\zeta+\left(x_{r}^{0}-x_{m}^{0}\right)_{0}}\{ & a_{2}+\frac{\kappa^{*}}{\alpha_{s}}\left(T_{m}-1\right)^{2} e^{\zeta+\left(x_{r}^{0}-x_{m}^{0}\right)_{0}} \\
& \left.-\frac{\alpha_{s}}{1-\alpha_{s}}\left[\hat{l}^{*}-\hat{r}^{*} \hat{b}(1-\chi)\right]\left(T_{m}-1\right)\left[\zeta+\left(x_{r}^{0}-x_{m}^{0}\right)_{0}\right]\right\}
\end{aligned}
$$

where $a_{2}$ is to be found from matching with the inner solution. In particular, employing a $(2,2)$ matching procedure (cf. [21]), we first construct the two-term inner expansion of the two-term outer solution $q_{0}+\epsilon q_{1}$, expressed in terms of the inner variable $\hat{\eta}$, noting that

$$
q_{0} \sim T_{m}-1+\epsilon\left(T_{m}-1\right)\left[\hat{\eta}-\left(x_{r}^{0}-x_{m}^{0}\right)_{1}\right]+\cdots, \quad q_{1} \sim \frac{\kappa^{*}}{\alpha_{s}}\left(T_{m}-1\right)^{2}+a_{2}+\cdots
$$

where, in applying the definition (7.2) for $\hat{\eta}$, the expansion given by the first of Eqs. (7.1d) for $x_{r}^{0}-x_{m}^{0}$ has been used. Similarly, the outer expansion of the two-term inner solution $p_{0}+\epsilon p_{1}$, expressed in terms of the inner variable $\hat{\eta}$, is determined by the expansions for $p_{0}$ and $p_{1}$ given by

$$
p_{0} \sim\left(T_{m}-1\right)+\cdots, \quad p_{1} \sim \frac{\kappa^{*}}{\alpha_{s}}\left(T_{m}-1\right)\left(2 T_{m}-p_{b}-1\right)+\left(T_{m}-1\right) \hat{\eta}+\cdots
$$

Equating these two-term outer (inner) expansions of the the two-term inner (outer) solutions then determines that $a_{2}=\left(T_{m}-1\right)\left[\left(\kappa^{*} / \alpha_{s}\right)\left(T_{m}-p_{b}\right)+\left(x_{r}^{0}-x_{m}^{0}\right)_{1}\right]$, and thus the final solution for $q_{1}$ is given by

$$
\begin{aligned}
q_{1}= & \left(x_{r}^{0}-x_{m}^{0}\right)_{1}\left(T_{m}-1\right) e^{\zeta+\left(x_{r}^{0}-x_{m}^{0}\right)_{0}}+\frac{\kappa^{*}}{\alpha_{s}}\left(T_{m}-1\right) e^{\zeta+\left(x_{r}^{0}-x_{m}^{0}\right)_{0}}\left[T_{m}-p_{b}+\left(T_{m}-1\right) e^{\zeta+\left(x_{r}^{0}-x_{m}^{0}\right)_{0}}\right] \\
& -\frac{\alpha_{s}}{1-\alpha_{s}}\left[\hat{l}^{*}-\hat{r}^{*} \hat{b}(1-\chi)\right]\left(T_{m}-1\right)\left[\zeta+\left(x_{r}^{0}-x_{m}^{0}\right)_{0}\right] e^{\zeta+\left(x_{r}^{0}-x_{m}^{0}\right)_{0}}, \quad \zeta<-\left(x_{r}^{0}-x_{m}^{0}\right)_{0} .
\end{aligned}
$$


The perturbation analysis of Eq. (A.10) can be continued, at least in principle, to determine higher-order coefficients in the expansion of the outer pressure variable in the solid-gas region. In the present stability analysis, however, knowledge of the coefficients $q_{0}$ and $q_{1}$ is sufficient to determine both the leading-order growth rate $i \omega_{0}$ and the first-order correction coefficient $i \omega_{1}$.

\section{APPENDIX B. Calculation of $d \nu^{(1)} / d \zeta, \sigma^{(2)}$ and $d \sigma_{b l}^{(0)} /\left.d \hat{\eta}\right|_{\hat{\eta}=0}$}

The coefficients $N_{i}$ and $S_{i}$ appearing in the solutions (9.2) and (9.4) for $\nu^{(1)}$ and $\sigma^{(2)}$, respectively, are tabulated here for completeness. In, particular, Eq. (6.14) at $\mathrm{O}(\epsilon)$ gives an expression for $\nu^{(1)}$ as

$$
\nu^{(1)}=\sigma^{(1)}+\frac{\kappa^{*}}{\alpha_{s}}\left(q_{0}+1\right) \frac{d \nu^{(0)}}{d \zeta}+\frac{\kappa^{*}}{\alpha_{s}} \frac{d q_{0}}{d \zeta} \nu^{(0)}+i \omega_{0} \frac{\kappa^{*}}{\alpha_{s}}\left(q_{0}+1\right) \frac{d q_{0}}{d \zeta}, \quad \zeta<-\left(x_{r}^{0}-x_{m}^{0}\right)_{0},
$$

where the right-hand side is known from the previously calculated lower-order results for $\nu^{(0)}=$ $\sigma^{(0)}, \sigma^{(1)}$ and $q_{0}$ given by Eqs. (8.3) and (8.20), Eqs. (8.5a,b,c) and Eq. (A.12), respectively. Consequently, we obtain the result (9.2) for $d \nu^{(1)} / d \zeta$, where the $N_{i}$ are given by

$$
\begin{aligned}
& N_{1}=q_{+}^{(0)} \hat{c}_{1}+N_{2} / q_{+}^{0}-\frac{\kappa^{*}}{\alpha_{s}} c_{m}^{(0)}\left(T_{m}-1\right)\left(q_{+}^{0}\right)^{2} e^{q_{+}^{0}\left(x_{r}^{0}-x_{m}^{0}\right)_{0}} \\
& N_{2}=\frac{q_{+}^{0}\left(T_{m}-1\right) c_{m}^{(0)}}{2 q_{+}^{(0)}-1}\left\{\frac{\alpha_{s}}{1-\alpha_{s}}\left[\hat{l}^{*}\left(i \omega_{0}+q_{+}^{(0)}\right)+\hat{r}^{*} \hat{b}(\chi-1) q_{+}^{(0)}\right]-i \omega_{1}\right\} e^{q_{+}^{0}\left(x_{r}^{0}-x_{m}^{0}\right)_{0}} \\
& N_{3}=i \omega_{0}\left[\frac{\kappa^{*}}{\alpha_{s}}-\frac{\hat{r}^{*} \hat{b}(\chi-1) \alpha_{s}}{\left(1-\alpha_{s}\right)\left(i \omega_{0}+k^{2}\right)}\right]\left(T_{m}-1\right) e^{\left(x_{r}^{0}-x_{m}^{0}\right)_{0}} \\
& N_{4}=2 i \omega_{0} \frac{\kappa^{*}}{\alpha_{s}}\left(T_{m}-1\right)^{2} e^{2\left(x_{r}^{0}-x_{m}^{0}\right)_{0}} \\
& N_{5}=\frac{\kappa^{*}}{\alpha_{s}} c_{m}^{(0)}\left(T_{m}-1\right)^{2}\left(q_{+}^{0}+1\right)^{2} e^{\left(1+q_{+}^{0}\right)\left(x_{r}^{0}-x_{m}^{0}\right)_{0}}
\end{aligned}
$$

Similarly, the right-hand side of Eq. (9.4) for $\sigma^{(2)}$ is known from the previous results for $\sigma^{(1)}$, $d \nu^{(1)} / d \zeta$ and the basic quantities $T_{0}, T_{1}, q_{0}$ and $q_{1}$, where the last of these is given by Eq. (A.19). As a result, Eq. (9.3) can be written in the form

$$
\frac{d^{2} \sigma^{(2)}}{d \zeta^{2}}-\frac{d \sigma^{(2)}}{d \zeta}-\left(i \omega_{0}+k^{2}\right) \sigma^{(2)}+\hat{L}_{1} e^{q_{+}^{0} \zeta}+\hat{L}_{2} \zeta e^{q_{+}^{\mathrm{a} \zeta}}+\hat{L}_{3} e^{\zeta}+\hat{L}_{4} \zeta e^{\zeta}+\hat{L}_{5} e^{2 \zeta}+\hat{L}_{6} e^{\left(q_{+}^{\mathrm{o}}+1\right) \zeta},
$$

for $\zeta<-\left(x_{r}^{0}-x_{m}^{0}\right)_{0}$, which has the solution (9.4) with the $S_{i}$ given by

$$
\begin{aligned}
& S_{1}=-\frac{\hat{L}_{1}}{2 q_{+}^{0}-1}+\frac{\hat{L}_{2}}{\left(2 q_{+}^{0}-1\right)^{2}}, \quad S_{2}=-\frac{\hat{L}_{2}}{2\left(2 q_{+}^{0}-1\right)}, \quad S_{3}=\frac{\hat{L}_{3}+\hat{L}_{4}}{i \omega_{0}+k^{2}}, \\
& S_{4}=-\frac{\hat{L}_{4}}{i \omega_{0}+k^{2}}, \quad S_{5}=\frac{\hat{L}_{5}}{2-i \omega_{0}-k^{2}}, \quad S_{6}=\frac{\hat{L}_{6}}{q_{+}^{0}\left(q_{+}^{0}+1\right)-i \omega_{0}-k^{2}},
\end{aligned}
$$


where

$$
\begin{aligned}
& \hat{L}_{1}=-i \omega_{1} \hat{c}_{1}+i \omega_{2} c_{m}^{(0)}\left(T_{m}-1\right) e^{q_{+}^{0}\left(x_{r}^{0}-x_{m}^{0}\right)_{0}}-\frac{\alpha_{s}}{1-\alpha_{s}}\left\{\hat{r}^{*} \hat{b}\left(\hat{c}_{1}-\frac{L_{1}}{2 q_{+}^{0}-1}-\chi N_{1}\right)\right. \\
& -\hat{l}^{*}\left[\hat{c}_{1}\left(q_{+}^{0}-i \omega_{0}\right)+\left(i \omega_{1}-\frac{\alpha_{s}}{1-\alpha_{s}}\left[\hat{r}^{*} \hat{b}(\chi-1) q_{+}^{0}+\hat{l}^{*}\left(i \omega_{0}+q_{+}^{0}\right)\right]\right) c_{m}^{(0)}\left(T_{m}-1\right) e^{\left.\left.q_{+}^{0}\left(x_{r}^{0}-x_{m}^{0}\right)_{0}\right]\right\}},\right. \\
& \hat{L}_{2}=\frac{i \omega_{1} L_{1}}{2 q_{+}^{0}-1}+\frac{\alpha_{s}}{1-\alpha_{s}}\left[\hat{r}^{*} \hat{b}\left(\frac{q_{+}^{0} L_{1}}{2 q_{+}^{0}-1}+\chi N_{2}\right)-\hat{l}^{*} \frac{\left(i \omega_{0}+q_{+}^{0}\right) L_{1}}{2 q_{+}^{0}-1}\right] \\
& \hat{L}_{3}=-\frac{i \omega_{1} L_{2}}{i \omega_{0}+k^{2}}+\frac{\alpha_{s}}{1-\alpha_{s}}\left\{\hat{l}^{*}\left[\frac{\left(i \omega_{0}+1\right) L_{2}}{i \omega_{0}+k^{2}}+\frac{\hat{r}^{*} \hat{b}(\chi-1) \alpha_{s}}{1-\alpha_{s}} i \omega_{0}\left(T_{m}-1\right) e^{\left(x_{r}^{0}-x_{m}^{0}\right\rangle_{0}}\right]\right. \\
& \left.+\hat{r}^{*} \hat{b}\left[-\frac{L_{2}}{i \omega_{0}+k^{2}}+\chi\left(N_{3}+N_{4}\right)\right]\right\} \\
& +\frac{\hat{r}^{*} \hat{b} \alpha_{s}}{1-\alpha_{s}}\left\{i \omega_{0}(1-\chi)\left[\left(x_{r}^{0}-x_{m}^{0}\right)_{1}+\frac{\kappa^{*}}{\alpha_{s}}\left(T_{m}-p_{b}\right)-\frac{\alpha_{s}}{1-\alpha_{s}}\left[\hat{l}^{*}-\hat{r}^{*} \hat{b}(1-\chi)\right]\left[\left(x_{r}^{0}-x_{m}^{0}\right)_{0}+1\right]\right]\right. \\
& \left.+\hat{r}^{*} \hat{b}\left[i \omega_{1}(1-\chi)-i \omega_{0} \frac{\kappa^{*}}{\alpha_{s}}\right]\right\}\left(T_{m}-1\right) e^{\left(x_{r}^{0}-x_{m}^{o}\right)_{0}} \\
& \hat{L}_{4}=\frac{\hat{r}^{*} \hat{b} \alpha_{s}}{1-\alpha_{s}} i \omega_{0}(1-\chi)\left[\hat{l}^{*}-\hat{r}^{*} \hat{b}(1-\chi)\right]\left(T_{m}-1\right) e^{\left(x_{r}^{0}-x_{m}^{0}\right)_{0}} \\
& \hat{L}_{5}=-2 \frac{\hat{r}^{*} \hat{b} \chi \alpha_{s}}{1-\alpha_{s}} i \omega_{0} \frac{\kappa^{*}}{\alpha_{s}}\left(T_{m}-1\right)^{2} e^{2\left(x_{r}^{0}-x_{m}^{0}\right)_{0}} \text {, } \\
& \hat{L}_{6}=\frac{\hat{r}^{*} \hat{b} \chi \alpha_{s}}{1-\alpha_{s}} N_{5}
\end{aligned}
$$

with $L_{1}$ and $L_{2}$ given by Eqs. (8.5b,c).

Finally, the calculation of the expression (9.9) for $d \sigma_{b l}^{(2)} /\left.d \eta\right|_{\hat{\eta}=0}$ is obtained by first considering the solution of Eq. (9.8) for $\sigma_{b l}^{(2)}$ itself. In particular, Eq. (9.8) admits the solution

$$
\begin{aligned}
& \sigma_{b l}^{(2)}=D_{1} \hat{\eta}+D_{2}-\frac{1}{2} c_{m}^{(0)}\left(T_{m}-1\right)\left(q_{+}^{0}\right)^{2} \hat{\eta}^{2}-\frac{\hat{l}^{*} \alpha_{s}}{1-\alpha_{s}}\left\{-c_{m}^{(0)}\left(T_{m}-1\right) q_{+}^{0}\left[\hat{\eta}-\left(x_{r}^{0}-x_{m}^{0}\right)_{1}\right]\right. \\
& +\left[\hat{c}_{1} e^{-q_{+}^{0}\left(x_{r}^{0}-x_{m}^{0}\right)_{0}}-c_{m}^{(0)}\left(T_{m}-1\right)\left(\frac{\alpha_{s}}{1-\alpha_{s}}\left[\hat{l}^{*}\left(i \omega_{0}+q_{+}^{0}\right)+\hat{r}^{*} \hat{b}(\chi-1) q_{+}^{0}\right]-i \omega_{1}\right) \frac{\left(x_{r}^{0}-x_{m}^{0}\right)_{0}}{2 q_{+}^{0}-1}\right] \\
& \left.-\frac{\hat{r}^{*} \hat{b} \alpha_{s}}{1-\alpha_{s}}\left[\left(T_{m}-1\right)(\chi-1) \frac{i \omega_{0}}{i \omega_{0}+k^{2}}-c_{m}^{(0)} \chi\left(p_{0}-T_{m}+1\right)\right]\right\} \\
& -\frac{\hat{r}^{*} \hat{b} \chi \kappa^{*}}{1-\alpha_{s}}\left\{c _ { m } ^ { ( 0 ) } T _ { m } ( T _ { m } - 1 ) \left[\frac{p_{b}+1}{p_{0}+1}\left(1-\frac{2 T_{m}-p_{b}-1}{p_{b}-T_{m}+1}\right)+\ln \left(p_{0}-T_{m}+1\right)\right.\right. \\
& \left.-\frac{3 T_{m}-p_{b}-1}{p_{0}+1} \ln \left(\frac{p_{0}-T_{m}+1}{p_{b}-T_{m}+1}\right)+\frac{p_{0}-T_{m}+1}{2\left(p_{0}+1\right)} \ln ^{2}\left(\frac{p_{0}-T_{m}+1}{p_{b}-T_{m}+1}\right)\right] \\
& -\left[i \omega_{0}-\left(T_{m}-1\right) c_{m}^{(0)}+\frac{\alpha_{s}}{\kappa^{*}} c_{m}^{(1)}\right] p_{0}-c_{m}^{(0)}\left(T_{m}-1\right)\left(p_{b}-T_{m}+1\right) \ln \left(p_{0}-T_{m}+1\right) \\
& \left.+\left(c_{m}^{(0)}+i \omega_{0}\right)\left(\frac{1}{2} p_{0}^{2}+T_{m}\left[p_{0}+\left(T_{m}-1\right) \ln \left(p_{0}-T_{m}+1\right)\right]\right)\right\}
\end{aligned}
$$


where the integration constants $D_{1}$ and $D_{2}$ are determined from matching with the outer solution. To this end, the behavior of the inner solution given above for $\hat{\eta} \rightarrow-\infty$ is, since $p_{0} \sim\left(T_{m}-1\right)+$ $e^{\alpha_{s} \hat{\eta} / T_{m} \kappa^{*}}$ in that limit,

$$
\begin{gathered}
\sigma_{b l}^{(2)} \sim\left(T_{m}-1\right)\left\{-\frac{c_{m}^{(0)}}{2}\left(q_{+}^{0} \hat{\eta}\right)^{2}+\frac{\alpha_{s}}{1-\alpha_{s}}\left[\hat{l}^{*} q_{+}^{0} c_{m}^{(0)}+\hat{r}^{*} \hat{b} \chi\left(c_{m}^{(0)}+\frac{c_{m}^{(0)}+i \omega_{0}}{T_{m}}\right)\right] \hat{\eta}\right\} \\
+D_{1} \hat{\eta}+D_{2}+\cdots
\end{gathered}
$$

In addition, we calculate the behavior of the outer solution $\sigma^{(2)}$ given above in the limit $\zeta \rightarrow$ $-\left(x_{r}^{0}-x_{m}^{0}\right)$ as

$$
\begin{aligned}
\left.\sigma^{(2)}\right|_{\zeta=-\left(x_{r}^{0}-x_{m}^{0}\right)} \sim & {\left[\tilde{c}_{1}-S_{1}\left(x_{r}^{0}-x_{m}^{0}\right)_{0}+S_{2}\left(x_{r}^{0}-x_{m}^{0}\right)_{0}^{2}\right] e^{-q_{+}^{o}\left(x_{r}^{0}-x_{m}^{0}\right)_{0}} } \\
& +\left[S_{3}-S_{4}\left(x_{r}^{0}-x_{m}^{0}\right)_{0}\right] e^{-\left(x_{r}^{0}-x_{m}^{0}\right)_{0}}+S_{5} e^{-2\left(x_{r}^{0}-x_{m}^{0}\right)_{0}}+S_{6} e^{-\left(1+q_{+}^{0}\right)\left(x_{r}^{0}-x_{m}^{0}\right)_{0}} .
\end{aligned}
$$

Performing similar calculations, to the appropriate orders in $\epsilon$, for the lower-order coefficients $\sigma^{(0)}, \sigma^{(1)}, \sigma_{b l}^{(0)}$ and $\sigma_{b l}^{(1)}$ given in the main body of the text, we may then perform a $(3,3)$ matching of the three-term inner and outer solutions $\sigma_{b l} \approx \sigma_{b l}^{(0)}+\epsilon \sigma_{b l}^{(1)}+\epsilon^{2} \sigma_{b l}^{(2)}$ and $\sigma \approx \sigma^{(0)}+\epsilon \sigma^{(1)}+\epsilon^{2} \sigma^{(2)}$, respectively. As a result, an expression for $D_{1}$ is obtained as

$$
\begin{aligned}
D_{1}= & {\left[\hat{c}_{1} q_{+}^{0}+L_{1} \frac{q_{+}^{0}\left(x_{r}^{0}-x_{m}^{0}\right)_{0}-1}{2 q_{+}^{0}-1}\right] e^{-q_{+}^{0}\left(x_{r}^{0}-x_{m}^{0}\right)_{0}}+\frac{L_{2}}{i \omega_{0}+k^{2}} e^{-\left(x_{r}^{0}-x_{m}^{0}\right)_{0}} } \\
& -\frac{\alpha_{s}\left(T_{m}-1\right)}{\left(1-\alpha_{s}\right) T_{m}}\left\{\hat{l}^{*} q_{+}^{0} c_{m}^{(0)} T_{m}+\hat{r}^{*} \hat{b} \chi\left[i \omega_{0}+c_{m}^{(0)}\left(T_{m}+1\right)\right]\right\}
\end{aligned}
$$

which enables $d \sigma_{b l}^{(2)} /\left.d \hat{\eta}\right|_{\hat{\eta}=0}$ to be calculated, giving the result (9.9). 


\section{Figure Captions}

Fig. 1a. Physical illustration of quasi-steady deflagration in a confined porous energetic material. The overpressure in the burned region tends to reverse the gas flow into, rather than out of, the unburned porous material. Consequently, for small permeabilities and $O(1)$ overpressures, a permeation boundary layer within the solid/gas region adjacent to the melting surface is established. Combustion occurs, for large activation energies, in an asymptotically thin reaction layer that separates the liquid/gas preheat zone and the burned-gas region.

Fig. 1b. The leading-order asymptotic form of the basic solution corresponding to quasi-steady, planar deflagration. The figure was drawn using the parameter values $p_{g}^{b}=p_{b}+1=6.0$, $T_{b}^{0}=6.0, \alpha_{s}=0.3, T_{m}=1.5, b=l=1.0, \kappa^{*}=1.0, \hat{r}^{*}=4.0$ and $\epsilon=0.025$. The boundary-layer character of the pressure and velocity profiles in the solid/gas region to the left of the meiting surface arises from the weak permeability of the porous solid.

Figs. 2a-f. Neutral stability boundaries $\beta^{0}(k)$, plotted as a function of disturbance wavenumber, for various values of overpressure $\left(p_{b}\right)$ and gas-to-solid ratios of heat capacity $(\hat{b})$ and scaled thermal conductivity $\left(\hat{l}^{*}\right)$. Modified Zel'dovich numbers that lie above (below) the stability boundary correspond to regions of instability (stability) with respect to nonsteady, nonplanar perturbations of the basic solution. The stability boundaries are calculated for representative values of the remaining parameters; specifically, $\alpha_{s}=0.3$, $T_{m}=1.5, T_{b}^{0}=6.0, \chi=0.3, \epsilon=0.1$.

Figs. 3a,b. Coefficients $\beta_{1}(k)$ and $\left(\beta_{1}+\hat{\beta}_{1}\right)(k)$ in the first-order correction to the leading-order stability boundary $\beta_{0}(k)$. These coefficients are calculated using the same representative values of the remaining parameters $\alpha_{s}, T_{m}, T_{b}^{0}$ and $\chi$ used in Figure 2. 


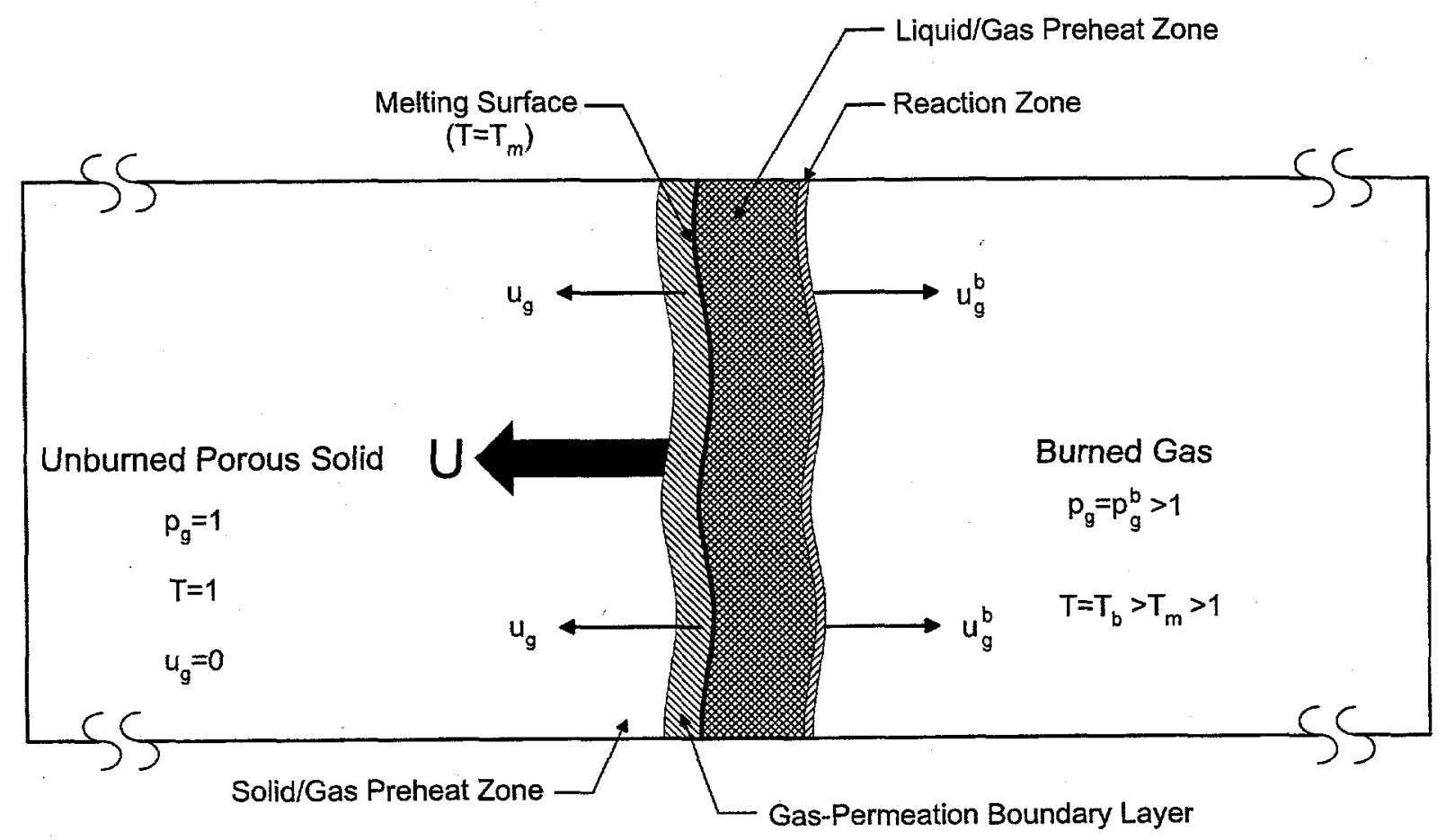

Figure 1a

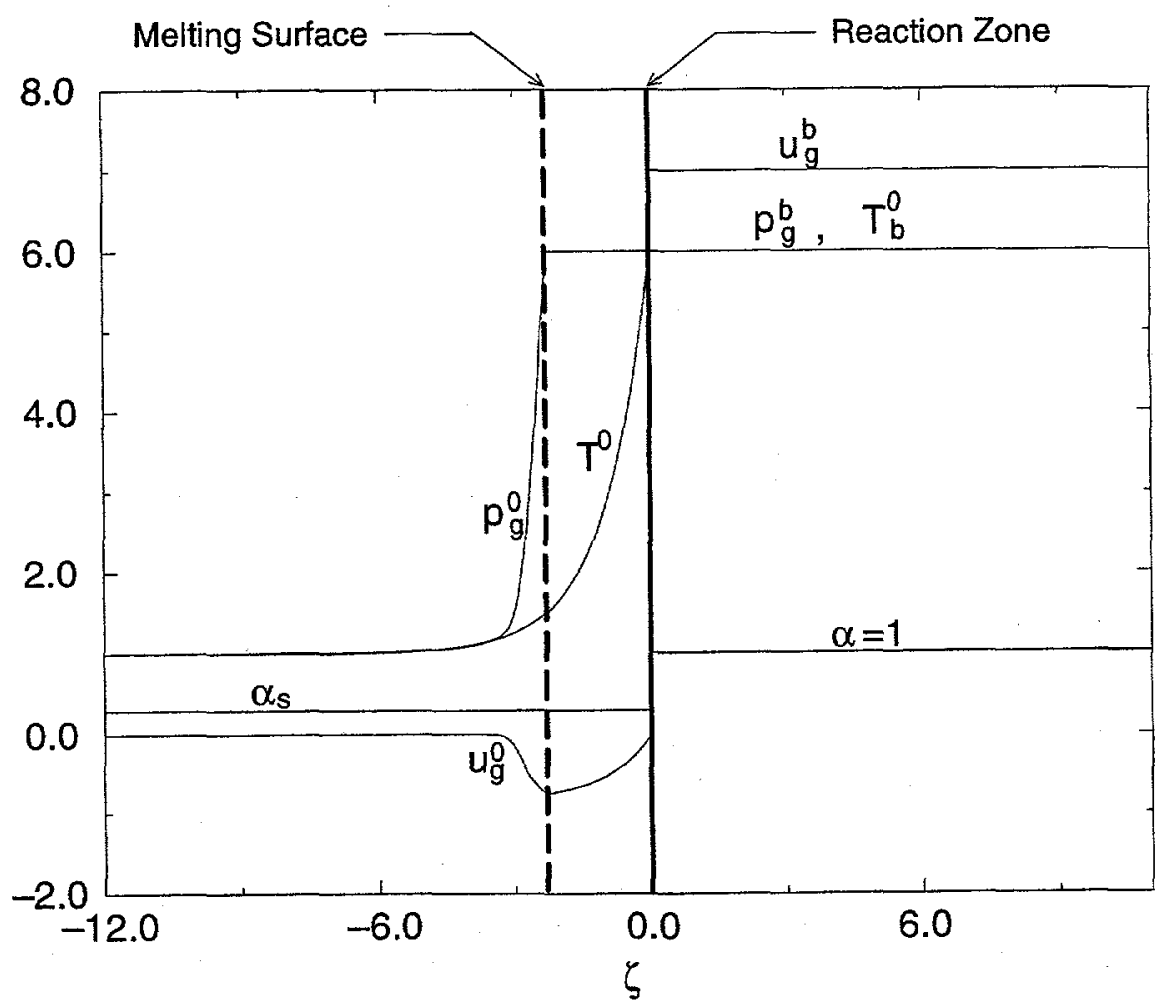

Figure 1b 


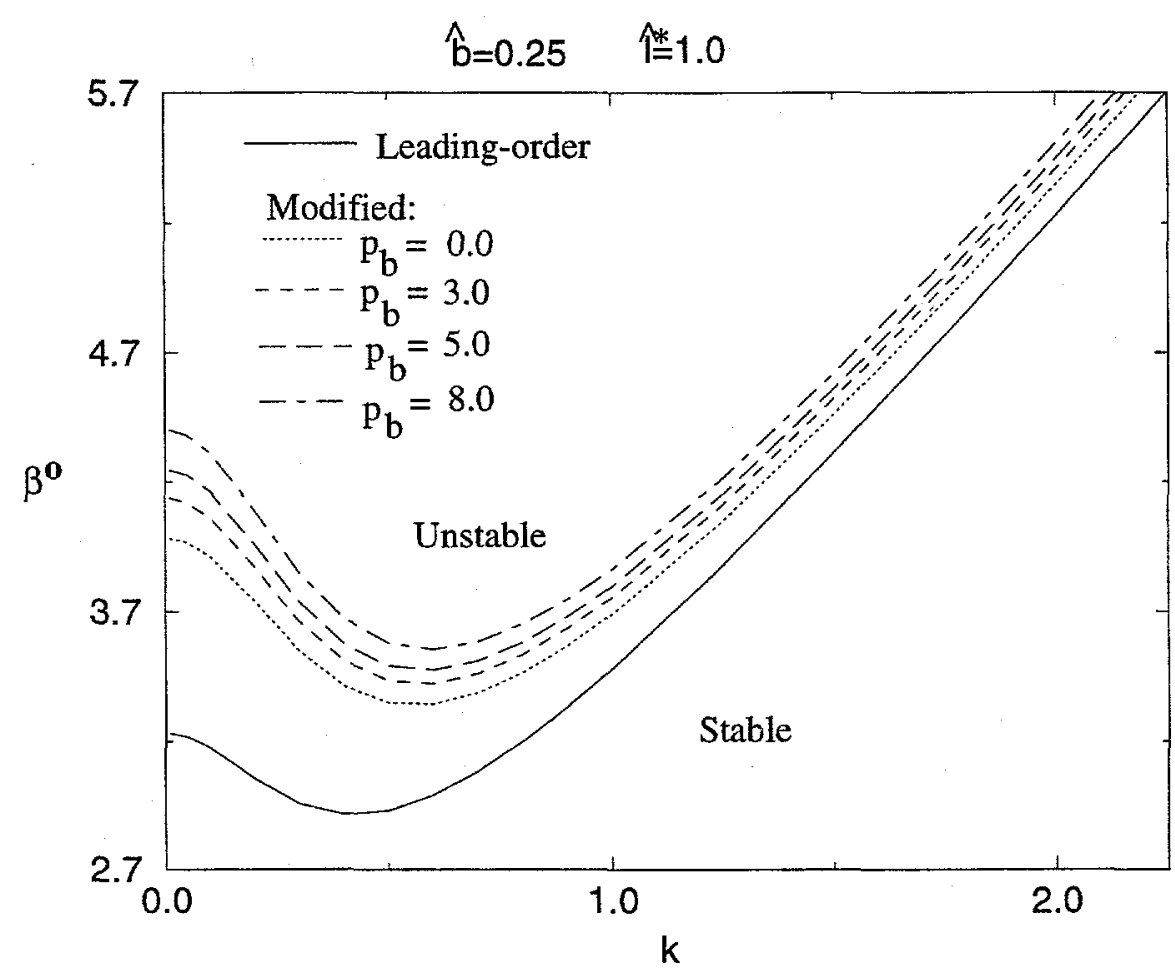

Figure 2a

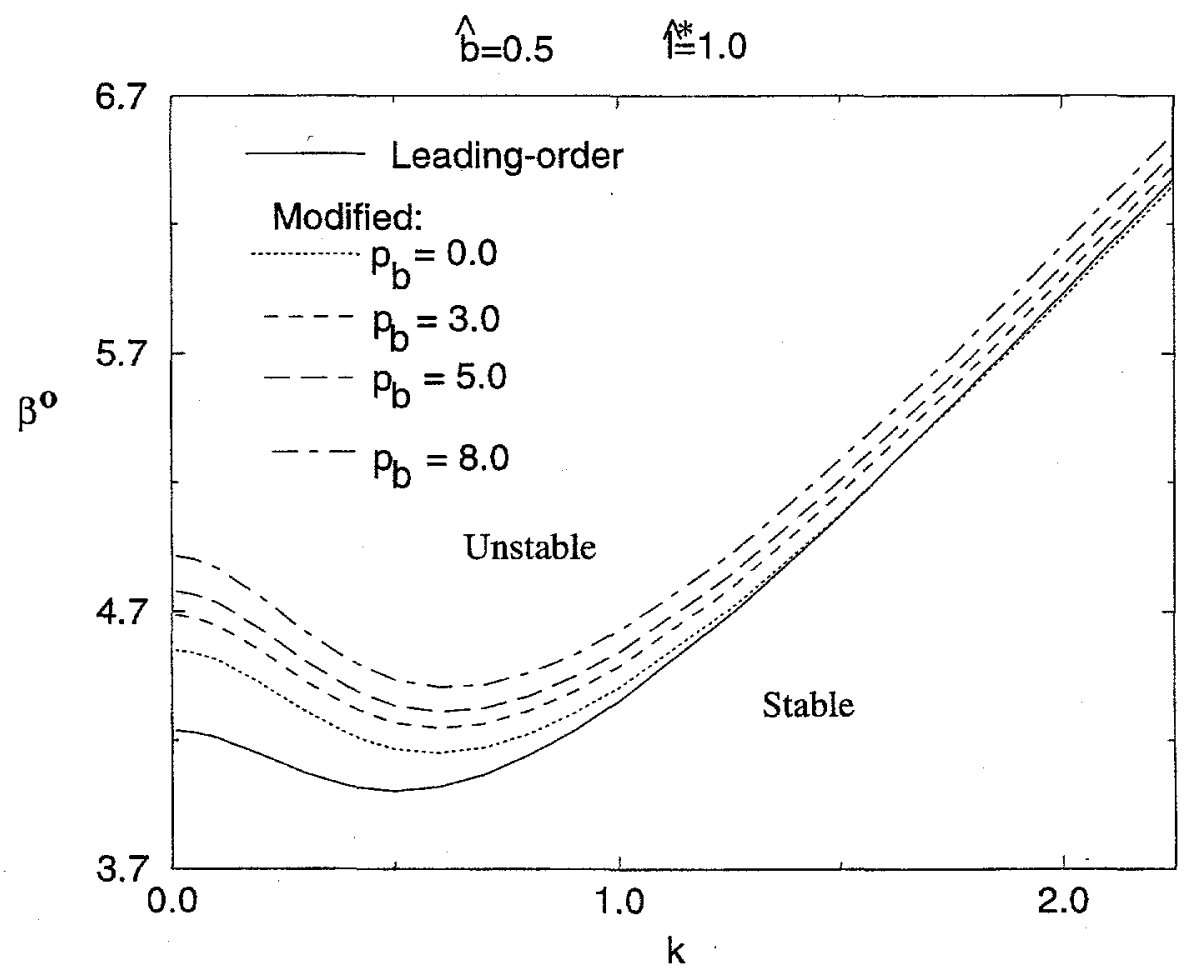

Figure 2b 


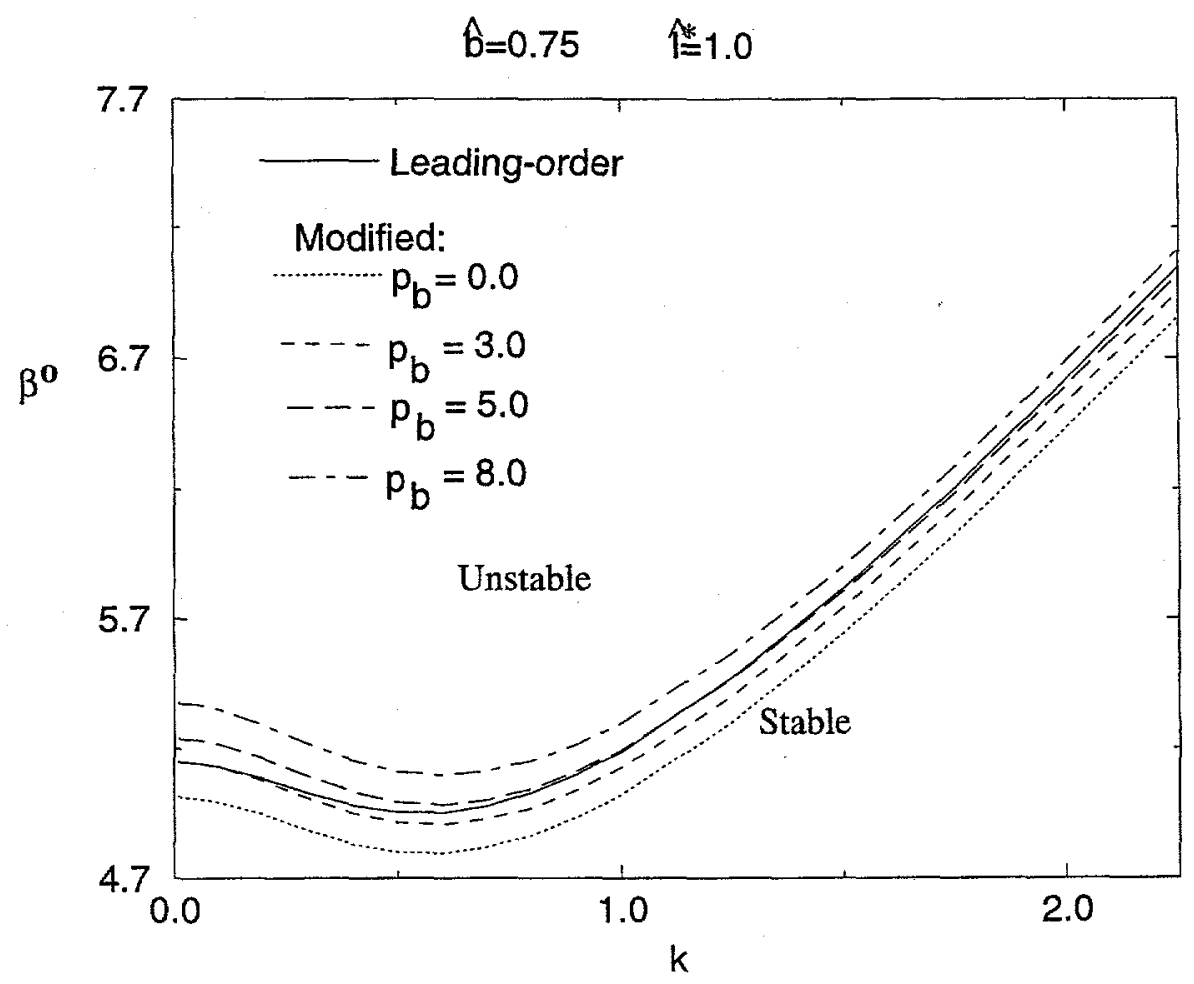

Figure 2c

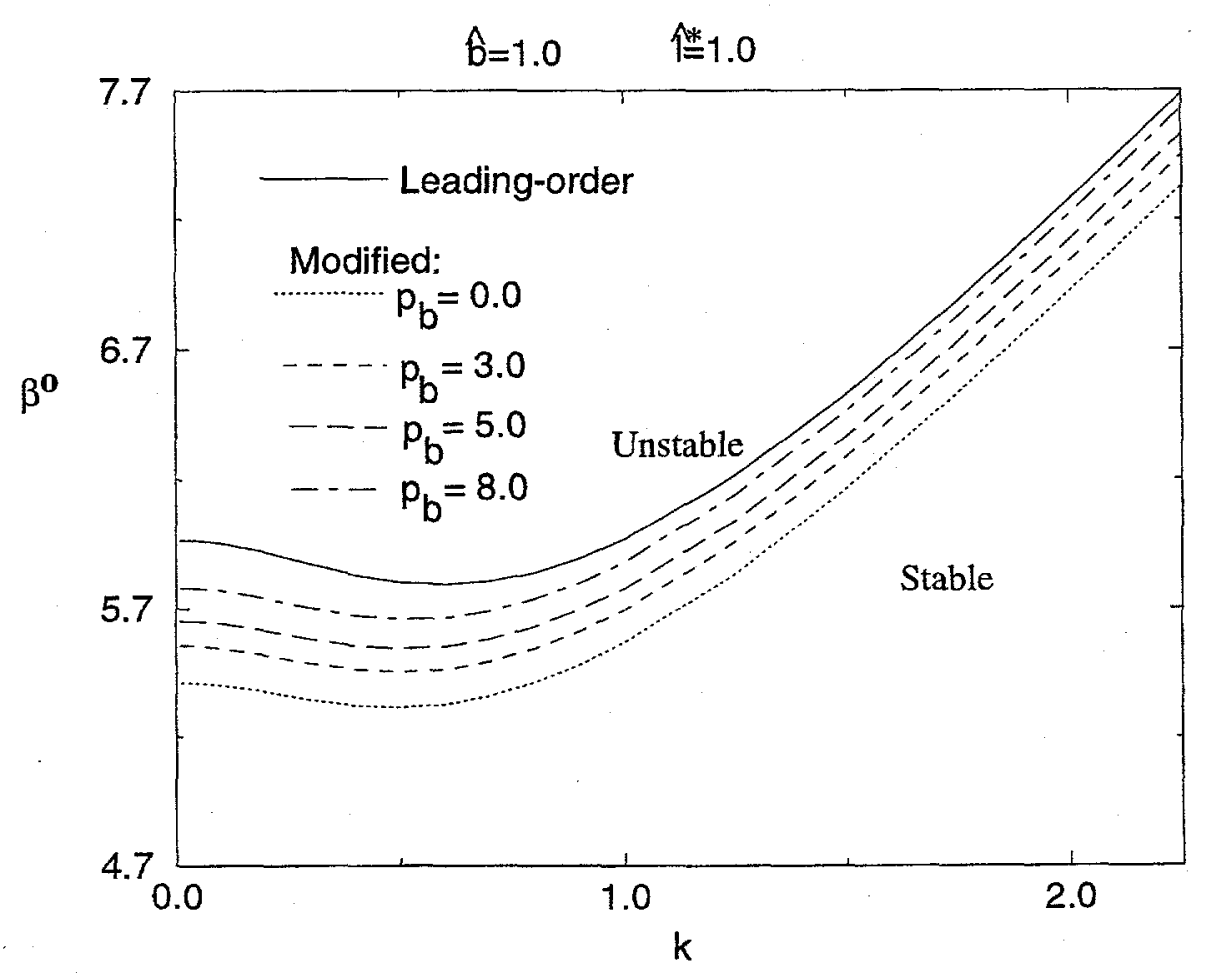

Figure 2d 


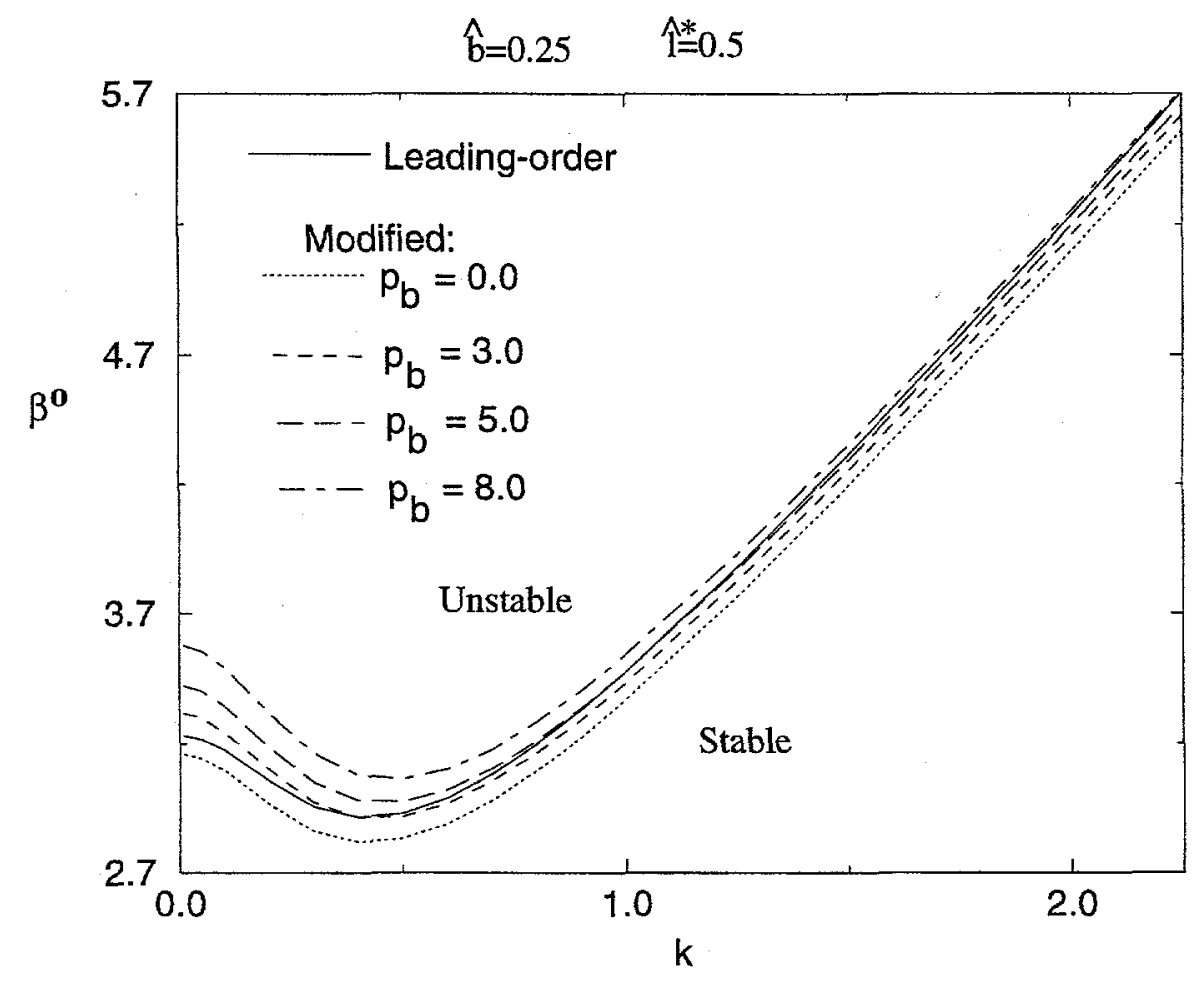

Figure 2e

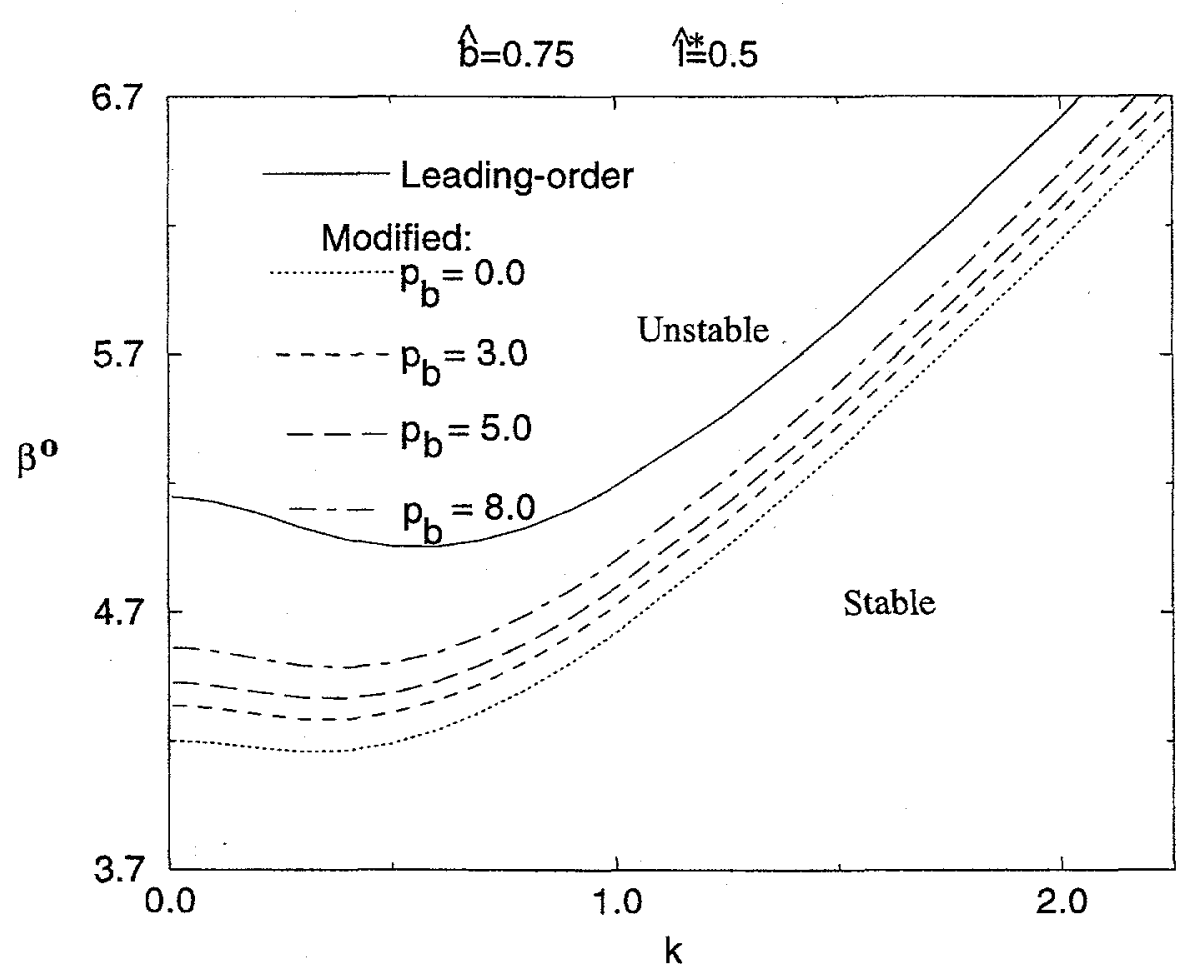

Figure $2 \mathrm{f}$ 


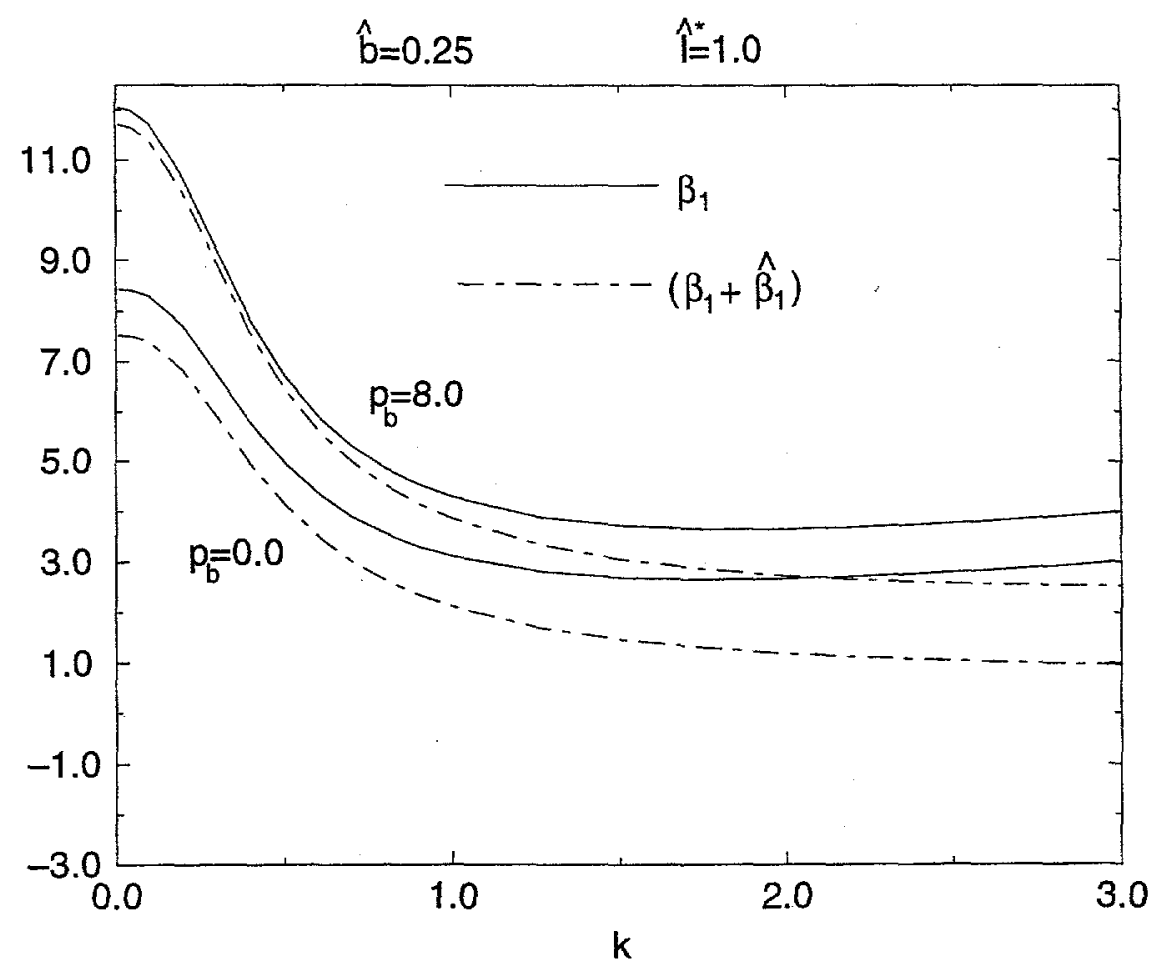

Figure $3 \mathrm{a}$

$\checkmark$

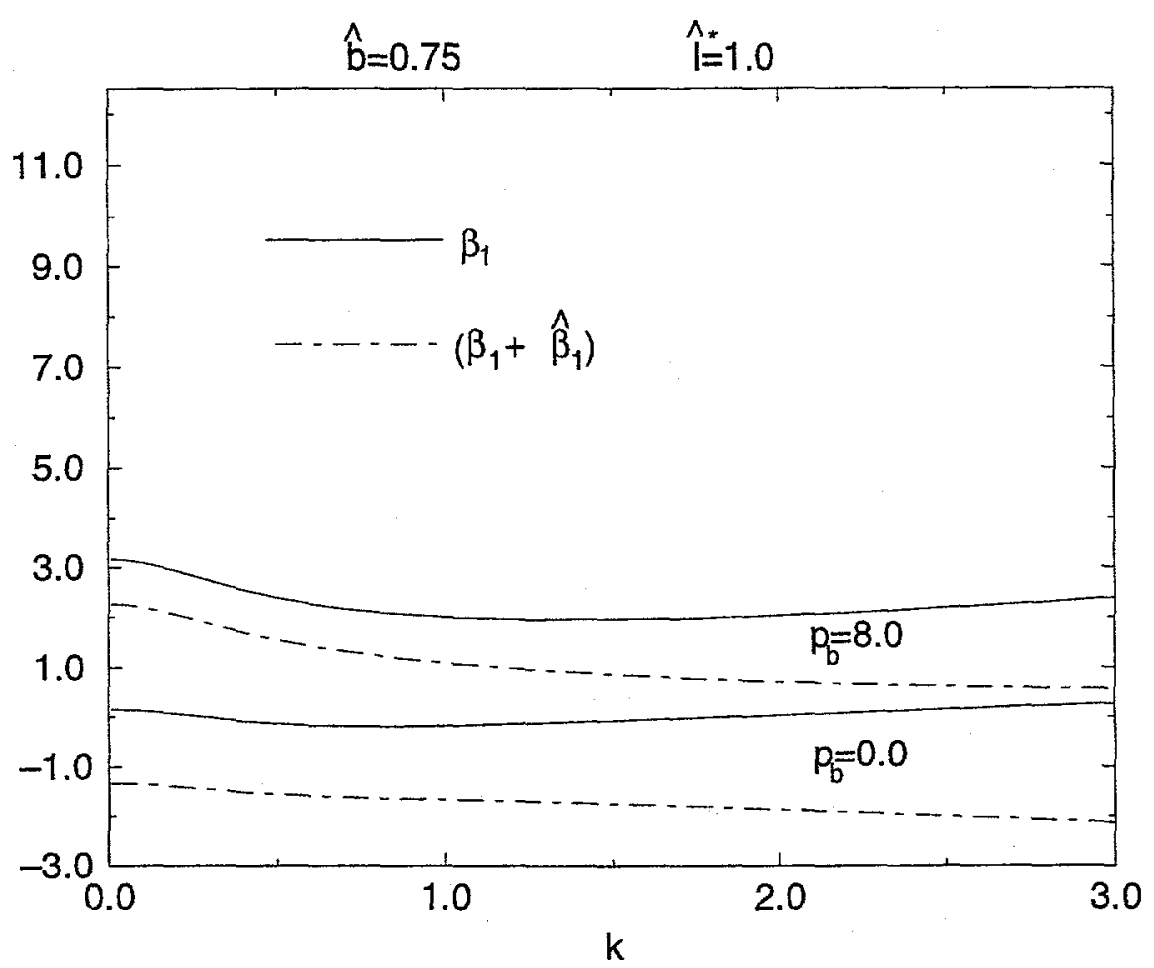

Figure $3 \mathrm{~b}$ 


\section{UNLIMITED RELEASE}

INITIAL DISTRIBUTION

Dr. John K. Bechtold

Department of Mathematics

New Jersey Institute of Technology

Newark, NJ 07102-1982

Dr. Mitat A. Birkan

Program Manager

Directorate of Aerospace and Engineering Sciences

Department of the Air Force

Bolling Air Force Base, DC 20332-6448

Prof. Michael Booty

Department of Mathematics

New Jersey Institute of Technology

Newark, NJ 07102-1982

Prof. John D. Buckmaster

Department of Aeronautical and Astronautical Engineering

University of Illinois

Urbana, IL 61801

Prof. Sebastien Candel

Ecole Central des Arts et Manufactures

Grande Voie de Vignes

92290 Chatenay-Malabry

FRANCE

Prof. J. F. Clarke

College of Aeronautics

Cranfield Institute of Technology

Cranfield-Bedford MK43 OAL

ENGLAND

Prof. Paul Clavin

Laboratoire Dynamique et Thermophysique des Fluides

Universite de Provence

Centre Saint Jerome

13397 Marseille Cedex 4

FRANCE

Prof. F. E. C. Culick

Jet Propulsion Center

California Institute of Technology

Pasadena, CA 91125

Prof. Martin Golubitsky

Department of Mathematics

University of Houston

University Park

Houston, TX 77004 
Prof. Michael Gorman

Department of Physics

University of Houston

Houston, TX 77004

Dr. Daryl D. Holm

CNLS, MS 457

Los Alamos National Laboratory

Los Alamos, NM 87545

Prof. G. M. Homsy

Department of Chemical Engineering

Stanford University

Stanford, CA 94305

Dr. G. Joulin

Laboratoirie D'Energetique et de Detonique

Universite de Poitiers

Rue Guillaume VII

86034 Poitiers

FRANCE

Dr. Hans Kaper

Applied Mathematics Division

Argonne National Laboratory

9700 S. Cass Ave.

Argonne, IL 60439

$\checkmark \quad$ Prof. A. K. Kapila

Department of Mathematical Sciences

Rensselaer Polytechnic Institute

Troy, NY 12128

Prof. D. R. Kassoy

Department of Mechanical Engineering

University of Colorado

Boulder, CO 80309

Prof. Joseph B. Keller

Department of Mathematics

Stanford University

Stanford, CA 94305

Prof. Barbara Keyfitz

Department of Mathematics

University of Houston

University Park

Houston, TX 77004

- Prof. C. K. Law

Department of Mechanical and Aerospace Engineering

Engineering Quadrangle

Princeton University

Princeton, NJ 08544 
Dr. Gary Leaf

Applied Mathematics Division

Argonne National Laboratory

9700 S. Cass Avenue

Argonne, IL 60439

Prof. Amable Liñán

Universidad Politecnica de Madrid

Escuela Tecnica Superior de Ingenieros Aeronauticos

Plaza del Cardenal Cisneros, 3

Madrid - 3

SPAIN

Prof. J. T. C. Liu

Division of Engineering, Box D

Brown University

Providence, RI 02912

Prof. Moshe Matalon

Department of Engineering Sciences and Applied Mathematics

Northwestern University

Evanston, IL 60208

Prof. Bernard J. Matkowsky

Department of Engineering Sciences and Applied Mathematics

Northwestern University

Evanston, IL 60208

Prof. A. C. McIntosh

Department of Fuel and Energy

University of Leeds

Leeds LS2 9JT

United Kingdom

Prof. D. O. Olagunju

Department of Mathematical Sciences

University of Delaware

Newark, DE 19716

Prof. R. E. O'Malley

Department of Applied Mathematics

University of Washington Seattle, WA 98195

Prof. Norbert Peters

Institute fur Allgemeine Mechanik

Technische Hochschule Aachen

Aachen

GERMANY

Prof. Victor Roytburd

Department of Mathematical Sciences

Rensselaer Polytechnic Institute

Troy, NY 12128 
Prof. W. A. Sirignano

School of Engineering

University of California, Irvine

Irvine, CA 92717

Prof. L. Sirovich

Division of Applied Mathematics, Box F

Brown University

Providence, RI 02912

Prof. G. I. Sivashinsky

Department of Mathematics

Tel-Aviv University

Ramat-Aviv, Tel-Aviv 69978

ISRAEL

Prof. Mitchell D. Smooke

Department of Mechanical Engineering

Yale University

New Haven, CT 06520

Prof. D. Scott Stewart

Department of Theoretical and Applied Mechanics

University of Illinois

Urbana, IL 61801

Prof. C. H. Su

Division of Applied Mathematics, Box F

Brown University

Providence, RI 02912

Prof. Cesar Treviño

Departmento de Termica y Fluidos

Universidad Nacional Autonoma de Mexico

Facultad de Ingenieria

Patios No. 12, Jardines del Sur

MEXICO 23, D.F.

Prof. Vladimir Volpert

Department of Engineering Sciences and Applied Mathematics

Northwestern University

Evanston, IL 60208

Prof. Forman A. Williams

Department of Applied Mechanics and Engineering Sciences

University of California, San Diego

La Jolla, CA 92093

Prof. Vigor Yang

Department of Mechanical Engineering

Pennsylvania State University

University Park, PA 16802 
Prof. Benn Zinn

Department of Aerospace Engineering

Georgia Institute of Technology

225 North Avenue, NW

Atlanta, GA 30332

C. K. Westbrook, LLNL, L-321

MS 0834 A. C. Ratzel, 9112

MS 0834 M. R. Baer, 9100

MS 0836 E. S. Hertel,9116

MS 0836 M. L. Hobbs, 9116

MS 0836 R. G. Schmitt, 9116

MS 9001 M. E. John, 8000

MS 9017 R. E. Stoltz, 4123

MS 9054 W. J. McLean, 8300

MS 9161 W. Bauer, 8358

MS 9405 C. M. Hartwig, 8700

MS 9056 L. A. Rahn, 8351

MS 9051 W. T. Ashurst, 8351

MS 9051 A. R. Kerstein, 8351

MS 9052 D. R. Hardesty, 8361

MS 9052 R. Behrens, 8361

MS 9052 S. B. Margolis, 8361 (30)

MS 9053 R. W. Carling, 8362

MS 9021 Technical Communications Department, 8815, for DOE/OSTI

MS 9021 Technical Communications Department, 8815/Technical Library, MS 0899, 4916

MS 0899 Technical Library, 4916

MS 9018 Central Technical Files, 8940-2 (3) 